Efficient Solution Methods for $\mathrm{N}$-component Condensation

D.S. van Putten 
Efficient Solution Methods for $N$-component Condensation D.S. van Putten

\section{Cover: D.S. van Putten}

Thesis University of Twente, Enschede - With summary in Dutch. ISBN 978-90-365-3265-5

Copyright (c) 2011 by D.S. van Putten, The Netherlands 


\title{
EFFICIENT SOLUTION METHODS FOR $N$-COMPONENT CONDENSATION
}

\author{
PROEFSCHRIFT
}

ter verkrijging van

de graad van doctor aan de Universiteit Twente,

op gezag van de rector magnificus, prof. dr. H. Brinksma,

volgens besluit van het College voor Promoties

in het openbaar te verdedigen

op vrijdag 14 oktober 2011 om 16.45 uur

door

Dennis Sebastian van Putten

geboren op 12 april 1982

te Hoogeveen 
Dit proefschrift is goedgekeurd door de promotor: prof. dr. ir. H.W.M. Hoeijmakers

en de assistent-promotor:

dr. ir. R. Hagmeijer 


\section{TABLE OF CONTENTS}

1 Introduction 1

1.1 Condensation phenomenon . . . . . . . . . . . . . . 1

1.2 Motivation . . . . . . . . . . . . . . . 2

1.3 Thesis outline ....................... 3

2 Equilibrium thermodynamics 5

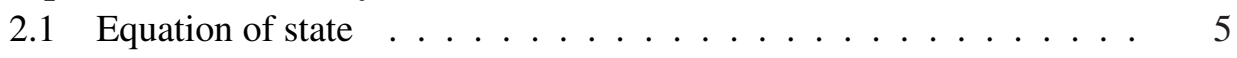

2.1.1 Single component fluid . . . . . . . . . . . 5

2.1.2 $N$-component fluid . . . . . . . . . . . . 6

2.2 Mixture equilibrium . . . . . . . . . . . . . . 6

2.3 Equilibrium computation . . . . . . . . . . . . 9

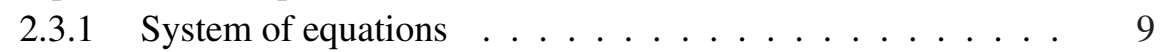

2.3.2 Numerical solution method . . . . . . . . . . . . . . 9 9

2.3 .3 Initialization . . . . . . . . . . . . . . . 10

2.4 Results . . . . . . . . . . . . . . . . . . . 10

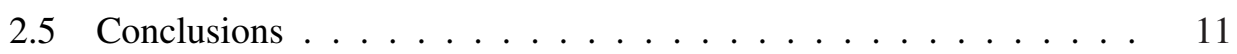

3 Models for $N$-component condensation $\quad 13$

3.1 Becker-Döring (NBD) equations . . . . . . . . . . . . 13

3.1.1 Equilibrium cluster size distribution . . . . . . . . . 15

3.2 Fokker-Planck Equation (NFPE) . . . . . . . . . . . . . . 17

3.3 General Dynamic Equation (NGDE) $\ldots \ldots \ldots \ldots$

4 A multigrid method for the NBD equations 21

4.1 Introduction . . . . . . . . . . . . . . . . . 21

$4.2 \quad N$-component Becker-Döring equations . . . . . . . . . . . 22

4.3 Numerical solution method . . . . . . . . . . . . . . . . . 23

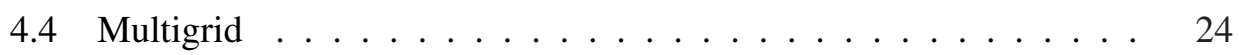

4.4.1 Coarse grid correction scheme . . . . . . . . . 25 
4.4.2 Geometrical coarsening . . . . . . . . . . . 26

4.4.3 Restriction and interpolation operators . . . . . . . . . . . 27

4.5 Results and discussion . . . . . . . . . . . . . 28

4.5.1 Steady state ternary nucleation . . . . . . . . . 28

4.5.2 Transient ternary nucleation . . . . . . . . . 31

4.6 Conclusions . . . . . . . . . . . . . . . . . . 34

5 Stationary Diffusion Flux model 37

5.1 Introduction . . . . . . . . . . . . . . . . . . . 37

5.2 Stationary Diffusion Flux model . . . . . . . . . . . . 38

5.3 Results and discussion . . . . . . . . . . . . . . . 42

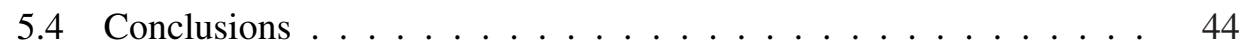

6 Phase Path Analysis algorithm for the NGDE 45

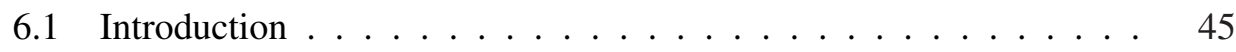

$6.2 N$-component General Dynamic Equation . . . . . . . . . . 46

6.2.1 Special case of $N=1 \ldots \ldots \ldots \ldots \ldots$

6.3 Phase Path Analysis . . . . . . . . . . . . . . . . . . 53

6.4 Test case definition . . . . . . . . . . . . . . . . 56

6.4.1 Binary nucleation pulse experiment $\ldots \ldots \ldots \ldots 6$

6.4.2 Quinary nucleation pulse experiment . . . . . . . 56

6.4 .3 Computational method . . . . . . . . . . . . . 57

6.5 Results and discussion . . . . . . . . . . . . . . . . 57

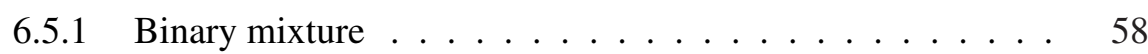

6.5 .2 Quinary mixture . . . . . . . . . . . . . 59

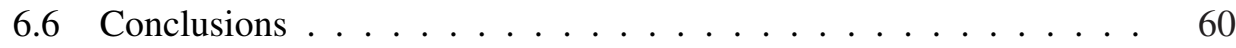

7 Conclusions and recommendations $\quad 67$

7.1 Conclusions . . . . . . . . . . . . . . . . . . . . . 67

7.2 Recommendations. . . . . . . . . . . . . . . . 68

$\begin{array}{ll}\text { References } & 71\end{array}$

A Equilibrium thermodynamics for SRK equation of state 79

A.1 Fugacity coefficient and chemical potential . . . . . . . . . . 79

A.2 Compressibility factor . . . . . . . . . . . . . . 80

A.3 Elements of the Jacobian . . . . . . . . . . . . . . . . 81

A.4 Derivatives of $K_{i}^{\alpha} \ldots \ldots \ldots \ldots \ldots \ldots \ldots \ldots$

B Thermo-physical properties $\quad 83$ 
TABLE OF CONTENTS

iii

C $\quad N$-component dynamics near the critical size 85

C.1 Growth rate vector . . . . . . . . . . . . . . . . . . 85

C.2 Nucleation flux integration . . . . . . . . . . . . . 85

C.3 Source point location . . . . . . . . . . . . . . 87

$\begin{array}{lr}\text { Samenvatting } & 89\end{array}$

$\begin{array}{lr}\text { Summary } & 91\end{array}$

Acknowledgment

About the author $\quad 95$ 



\section{INTRODUCTION}

This chapter constitutes a general introduction to the physics of condensation. We discuss the condensation physics encountered in nature and the importance of phase transitions for industrial applications. Finally, an outline of the thesis is given.

\subsection{Condensation phenomenon}

Phase transition of $\mathrm{N}$-component mixtures is of great importance to various areas of physics. Applications can be found in the fields of condensing vapors [20], cavitation [54], crystallization [18, 61], ferromagnetics [57], aerosol and atmospheric science [17], combustion science [41] and chemical processes [26]. In this thesis we will focus on the vapor to liquid phase transition.

Condensation occurs when a vapor departs from its equilibrium state due to e.g. a change in external conditions. This non-equilibrium state of the vapor is characterized by its supersaturation and is the potential to form the liquid phase. After a certain induction time the system will attain a new equilibrium state containing both phases [68].

In nature we encounter many types of phase transition. The most common phenomenon is atmospheric cloud formation and it is known that its $N$-component character is important for an accurate description of this condensation process [12]. This thesis, however, will focus on the condensation due to rapidly changing external conditions, e.g. fast expanding nozzle flows. The supersaturations achieved in these expansions are relatively high, typically of the order of $10-100$, yielding high cluster formation rates, i.e. high nucleation rates [73]. At these high rates the existence of foreign host particles is not important and the process can be regarded as homogeneous nucleation.

Condensation is often divided into the processes of nucleation and growth. Nucleation can be regarded as the formation of very small stable liquid clusters from the vapor molecules which occurs at microsecond time scales. The growth process is the subsequent stage where these clusters increase their size to form the stable liquid 
phase. Although this division is frequently used in literature the processes of nucleation and growth are coupled and the separation is merely a convenient terminology.

\subsection{Motivation}

The process of condensation can be exploited in natural gas applications. One of these applications is the Twister Supersonic Gas Separator, see Fig. 1.1. Natural gas consists of many components, e.g. alkanes, alcohols, inorganics and inert gas components. The Twister Supersonic Gas Separator utilizes a Laval nozzle to selectively condense specific components. These components can be either undesired (e.g. hydrogen-sulfide, mercury) or highly valued (e.g. heavy hydrocarbons). The working principle is as follows, see Fig. 1.1. The untreated saturated feed gas is supplied from the left and guided around the inner core. At the maximum diameter an array of guide vanes induces a swirl into the flow, and the flow is then accelerated and expanded through the Laval nozzle, resulting in: (i) the selective condensation of specific components because of the implied cooling rate and (ii) increase of the tangential velocity. The droplets are separated and collected at the outer wall thereby removing the undesired components from the inner gas stream. In most natural gas applications the treated dry gas stream in Fig. 1.1 consists mainly of methane.

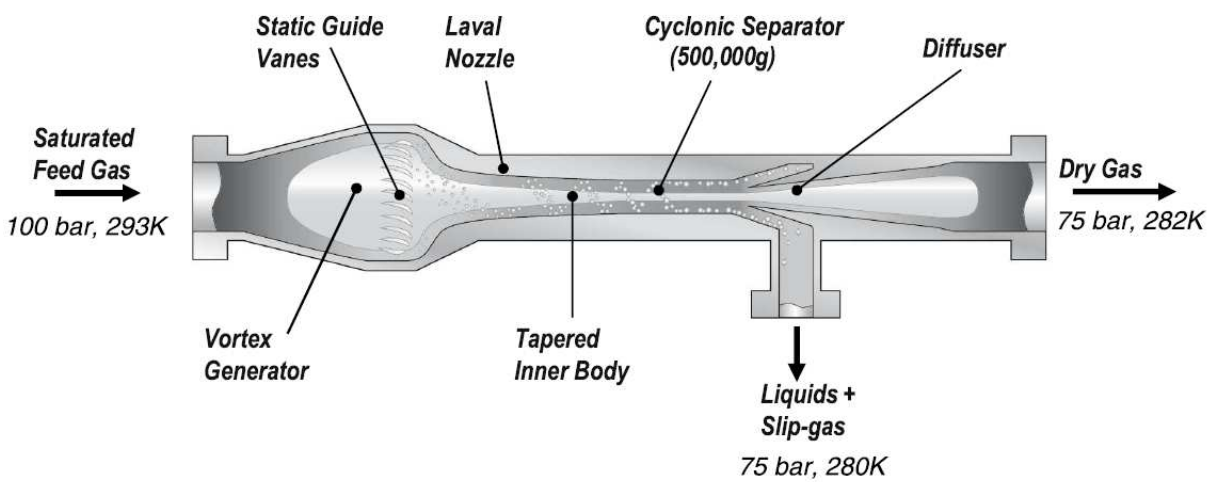

Figure 1.1: Schematic representation of a Twister Supersonic Gas Separator (image courtesy of Twister B.V.).

Understanding the $\mathrm{N}$-component condensation process is one of the main challenges in the development of the Twister device. The simulation of the flow field 
coupled with the equations that describe the condensation process requires enormous numerical effort, e.g. Refs. [10, 44, 65]. The aim of this thesis is to enhance the efficiency of the solution algorithms for the $N$-component condensation models.

\subsection{Thesis outline}

The thesis starts with a robust numerical solution method for calculation of high pressure real-gas equilibrium properties. These so-called flash calculations are performed on a three-phase natural gas mixture. Then, an elaborate overview of the models of condensation is given in Chapter 3. In Chapter 4 an efficient multigrid method is developed for numerically solving the $N$-component Becker-Döring equations. These equations can be regarded as the exact model and the solution can only be obtained in the small cluster domain. Chapter 5 considers the Stationary Diffusion Flux model for single component condensation which is capable of accurately describing cluster dynamics in the entire cluster size domain. Simplification of the $N$-component Becker-Döring equations leads to the $N$-component General Dynamic Equation in Chapter 6, which allows for a tremendous increase in efficiency making the numerical simulation of $\mathrm{N}$-component condensation possible. The thesis terminates with a summary and ideas for possible future work. 



\section{EQUILIBRIUM THERMODYNAMICS}

To determine the non-equilibrium processes of nucleation and droplet growth, the equilibrium state has to be calculated. The mixtures encountered in the gas industry consist mainly of alkanes, alcohols, inorganics and inert components. The present chapter will start by considering the equations of state and additional comments are made on the application to $\mathrm{N}$-component mixtures. Then, the computation of the three-phase equilibrium state is described and results for a real-gas ternary mixture are presented.

\subsection{Equation of state}

\subsubsection{Single component fluid}

The equation of state for a single component fluid provides the relation between the pressure, $p$, the molecular volume, $v$, and the temperature, $T$. As discussed in Refs. [40, 42], an equation of state which is capable of describing the various phases should at least be cubic in volume. A wide class of equations of state can be summarized in a general cubic form:

$$
p=\frac{k_{\mathrm{B}} T}{v-b}-\frac{a(T)}{\left(v^{2}+\kappa v+\lambda\right)},
$$

where $k_{\mathrm{B}}$ is the Boltzmann constant. The parameter $a$ is a measure for the attraction between molecules and $b$ is the covolume. The parameters $\kappa$ and $\lambda$ are specified in Table 2.1 for some commonly used cubic equations of state, i.e. Van der Waals (VdW), Soave-Redlich-Kwong (SRK) and Peng-Robinson (PR). The temperature dependence of $a$ differs for each equation of state and depends on the acentric factor $\omega$. Both $a$ and $b$ are functions of the critical pressure and critical temperature, $p_{c}$ and $T_{c}$, respectively. 


\begin{tabular}{llc}
\hline Equation of state & $\kappa$ & $\lambda$ \\
\hline VdW & 0 & 0 \\
SRK & $b$ & 0 \\
PR & $2 b$ & $-b^{2}$ \\
\hline
\end{tabular}

TABLE 2.1: Parameters $\kappa$ and $\lambda$ for the generalized cubic equation of state for the Van der Waals (VdW), Soave-Redlich-Kwong (SRK) and Peng-Robinson (PR) equation of state.

\subsection{2 $N$-component fluid}

For a mixture of $N$ components, the equation of state is modified by the appropriate averaging of the pure component parameters $a$ and $b$. In the limit case of a single component fluid the averaging should return the corresponding value for the pure substance. The most common mixing rule for a parameter $q$ is due to the one-fluid theory of Van der Waals [40] and is a function of the mole fraction of the phases, denoted by $\mathbf{x}=\left(x_{1}, \ldots, x_{N}\right)$ :

$$
q_{m}=\sum_{i=1}^{N} \sum_{j=1}^{N} x_{i} x_{j} q_{i j}
$$

where the subscript ' $m$ ' denotes the mixture property. It is noted that the mixing rule should be evaluated separately for each phase. The parameter, $q_{i j}$ for $j \neq i$, is obtained by either an arithmetic average or a geometric average, denoted by superscripts $a$ and $g$, respectively

$$
q_{i j}^{a}=\frac{\left(q_{i i}+q_{j j}\right)}{2}\left(1-l_{i j}\right), \quad q_{i j}^{g}=\sqrt{q_{i i} q_{j j}}\left(1-k_{i j}\right),
$$

where $l_{i j}$ and $k_{i j}$ are the binary interaction parameters and fitted to experimental data. In accordance with Ref. [40], the parameter $a$ is combined using the geometric rule and the parameter $b$ is calculated using the arithmetic combining rule. The binary interaction parameters for $b$ are often assumed zero, yielding

$$
a_{m}=\sum_{i=1}^{N} \sum_{j=1}^{N} x_{i} x_{j} \sqrt{a_{i} a_{j}}\left(1-k_{i j}\right), \quad b_{m}=\sum_{i=1}^{N} x_{i} b_{i} .
$$

\subsection{Mixture equilibrium}

The mixtures considered in this chapter establish a three-phase equilibrium, i.e. they consists of a vapor, a condensate liquid and an aqueous liquid phase, denoted by superscript $v, c$ and $a$, respectively. The condensate liquid will consist mainly of 
alkanes, whereas the aqueous liquid phase will contain most of the inorganics. The liquid phases are immiscible and differ in density.

The equilibrium of the mixture is attained if the phases are in mechanical, thermal and chemical equilibrium. The first two conditions for a flat interface result in equality of pressure and temperature

$$
p^{\alpha}=p, \quad T^{\alpha}=T, \quad \text { for } \quad \alpha=v, c, a .
$$

The chemical equilibrium is established by equality of the chemical potential $\mu_{i}$ of the phases, e.g. see Refs. [39, 42]:

$$
\mu_{i}^{v}\left(p, T, \mathbf{x}^{v}\right)=\mu_{i}^{c}\left(p, T, \mathbf{x}^{c}\right)=\mu_{i}^{a}\left(p, T, \mathbf{x}^{a}\right), \quad \text { for } \quad i=1,2, \ldots, N,
$$

where $\mathbf{x}^{v}=\left(x_{1}^{v}, \ldots, x_{N}^{v}\right), \mathbf{x}^{c}=\left(x_{1}^{c}, \ldots, x_{N}^{c}\right)$ and $\mathbf{x}^{a}=\left(x_{1}^{a}, \ldots, x_{N}^{a}\right)$ are the vapor, condensate and aqueous molar fractions, respectively, which are normalized to unity with respect to their corresponding phase:

$$
\sum_{i=1}^{N} x_{i}^{\alpha}=1, \quad \text { for } \quad \alpha=v, c, a .
$$

The equilibrium calculations are facilitated by the introduction of the fugacity, $f_{i}$, of component $i$ in the mixture. The fugacity and chemical potential are related by

$$
\mu_{i}=\mu_{i}^{\mathrm{ref}}+k_{\mathrm{B}} T \ln \left(\frac{f_{i}}{f_{i}^{\mathrm{ref}}}\right),
$$

where the superscript 'ref' denotes an arbitrary reference state. The fugacity can be considered as a modified partial pressure where the effect of non-ideality of the mixture is accounted for. In the vapor phase, the ideal gas (superscript 'ig') is chosen as a reference, yielding

$$
\mu_{i}^{v}=\mu_{i}^{\mathrm{ig}}+k_{\mathrm{B}} T \ln \left(\frac{f_{i}^{v}}{x_{i}^{v} p}\right) .
$$

The ratio $\phi_{i}^{v} \equiv f_{i}^{v} /\left(x_{i}^{v} p\right)$ is a measure of the deviation from ideality, which is called the fugacity coefficient of component $i$ in the mixture. For an ideal gas mixture $f_{i}^{v}=x_{i}^{v} p$, so that $\phi_{i}^{v}=1$.

Similar reasoning can be used for the condensate and the aqueous liquid phases, both denoted by superscript $l=c, a$ for convenience. The activity coefficient is defined as $\gamma_{i}^{l} \equiv f_{i}^{l} /\left(x_{i}^{l} f_{i}^{\text {is }}\right)$ and accounts for the non-ideality of the liquid solution, resulting in

$$
\mu_{i}^{l}=\mu_{i}^{\text {is }}+k_{\mathrm{B}} T \ln \gamma_{i}^{l},
$$


where superscript 'is' denotes the ideal liquid solution state. The chemical equilibrium condition in Eq. (2.6) is equivalent to the condition $f_{i}^{v}=f_{i}^{c}=f_{i}^{a}$, yielding

$$
\frac{x_{i}^{v}}{x_{i}^{c}}=\frac{\gamma_{i}^{c} f_{i}^{\mathrm{is}}}{\phi_{i}^{v} p} \equiv K_{i}^{c}, \quad \frac{x_{i}^{v}}{x_{i}^{a}}=\frac{\gamma_{i}^{a} f_{i}^{\mathrm{is}}}{\phi_{i}^{v} p} \equiv K_{i}^{a},
$$

where $K_{i}^{c}$ and $K_{i}^{a}$ are termed the equilibrium constants of component $i$. This result for the equilibrium is referred to as the " $\gamma / \phi$ approach" which is applied extensively to natural gas mixtures. For hydrocarbon mixtures both the vapor and condensate phases are accurately described by a cubic equation of state and therefore the fugacity coefficients can be evaluated for both phases. The vapor-condensate equilibrium constant becomes

$$
K_{i}^{c}=\frac{\phi_{i}^{c}}{\phi_{i}^{v}}
$$

where the fugacity coefficient for phase $\alpha$ is calculated using the cubic equation of state, see Ref. [39]:

$$
\ln \phi_{i}^{\alpha}=\int_{V}^{\infty}\left\{\left[\frac{\partial n Z^{\alpha}}{\partial n_{i}}\right]_{T, V^{\prime}, n_{j \neq i}}-1\right\} \frac{d V^{\prime}}{V^{\prime}}-\ln Z^{\alpha},
$$

where $Z \equiv p v / k_{\mathrm{B}} T$ is the compressibility factor, $n_{i}$ is the number of molecules in the entire system of component $i$ and $n=\sum_{i} n_{i}$. In Eq. (2.13), the integration is performed over the total volume $V=n v$. The difference in the vapor and condensate $\phi_{i}$ is determined by the difference in the compressibility factor due to the composition of the respective phase. The resulting expressions of the fugacity coefficients for the SRK equation of state are derived in Appendix A.

For the aqueous components we use the two-suffix activity coefficient model of Margules [42]:

$$
k_{\mathrm{B}} T \ln \gamma_{i}^{a}=\sum_{j=1}^{N} \sum_{k=1}^{N}\left(A_{j i}-\frac{1}{2} A_{j k}\right) x_{j}^{a} x_{k}^{a},
$$

where the binary coefficients $A_{i j}$ are fitted to experimental data. The ideal solution fugacity $f_{i}^{\text {is }}$ is determined by [69]

$$
f_{i}^{\mathrm{is}}=\phi_{i}^{s} p_{i}^{s} \exp \left(\frac{p-p_{i}^{s}}{\rho_{i}^{l} k_{\mathrm{B}} T}\right),
$$

where $\phi_{i}^{s}$ is the pure component fugacity coefficient at saturation pressure $p_{i}^{s}$. The exponential term is called the Poynting factor and contains the pure liquid number density $\rho_{i}^{l}$. 


\subsection{Equilibrium computation}

Solving the phase equilibrium problem is often referred to as a flash calculation. In the next sections we summarize the governing equations, provide a numerical solution method and present typical results.

\subsubsection{System of equations}

We start with the overall mass balance for each component

$$
z_{i}=x_{i}^{v} \mathcal{V}+x_{i}^{c} C+x_{i}^{a} \mathcal{A}, \quad \text { for } \quad i=1,2, \ldots, N,
$$

where $\mathcal{V}, C$ and $\mathcal{A}$ are the phase mole fractions of the vapor, condensate and aqueous phases, respectively, satisfying: $\mathcal{V}+C+\mathcal{A}=1$. The feed mole fractions $z_{i}$ are normalized (Eq. (2.7)) and specified. By using the equilibrium constants from Eq. (2.11) and the normalization constraints for the fractions for each phase in Eq. (2.7), a closed system of $3 N+2$ equations is obtained for equal number of unknowns $\mathbf{x}^{c}, \mathbf{x}^{a}, \mathbf{x}^{v}, \mathcal{V}$ and $C$ :

$$
\left.\begin{array}{r}
x_{i}^{v}-x_{i}^{c} K_{i}^{c} \\
x_{i}^{v}-x_{i}^{a} K_{i}^{a} \\
z_{i}-x_{i}^{v} \mathcal{V}-x_{i}^{c} \mathcal{C}-x_{i}^{a}(1-\mathcal{V}-\mathcal{C}) \\
\sum_{i=1}^{N} x_{i}^{a}-1 \\
\sum_{i=1}^{N} x_{i}^{v}-1
\end{array}\right\}=0 .
$$

The normalization constraint (2.7) for the condensate liquid fractions, $\mathbf{x}^{c}$, follows directly from summation of Eq. (2.16). Many equilibrium calculation algorithms use a reduced set of equations improving the efficiency of the method [36, 37]. This reduction, however, involves the addition of several constraints (e.g. the normalization conditions from Eq. (2.7)) which can distort the convergence of the numerical scheme.

\subsubsection{Numerical solution method}

The set of equations (2.17) can be solved numerically by means of the NewtonRaphson method, e.g. see Ref. [70]. The $3 N+2$ unknown variables are written in a single vector, $\boldsymbol{\xi}=\left(\mathbf{x}^{c}, \mathbf{x}^{a}, \mathbf{x}^{v}, \mathcal{V}, C\right)^{\mathrm{T}}$, and the system of algebraic equations $\mathbf{F}(\boldsymbol{\xi})=0$ consists of the equations given in Eq. (2.17). Provided that the Jacobian of the system, $\mathrm{J}$, is non-singular, the solution is obtained by iteration

$$
\boldsymbol{\xi}^{n+1}=\boldsymbol{\xi}^{n}-\mathbf{J}^{-1}\left(\xi^{n}\right) \mathbf{F}\left(\boldsymbol{\xi}^{n}\right),
$$

where the superscripts $n$ and $n+1$ indicate the current and the updated value of the solution, respectively. The $(k, l)^{\text {th }}$ element of the Jacobian is defined as

$$
\mathrm{J}_{k l}=\frac{\partial F_{k}}{\partial \xi_{l}} \quad \text { with } \quad k, l=1, \ldots, 3 N+2 .
$$


The Jacobian elements are evaluated in Appendix A.3. We note that for the first two equations of (2.17) we need the composition dependence of the equilibrium constants $K_{i}^{c}\left(\mathbf{x}^{c}, \mathbf{x}^{v}, p, T\right)$ and $K_{i}^{a}\left(\mathbf{x}^{a}, \mathbf{x}^{v}, p, T\right)$. The derivatives of $K_{i}^{c}$ with respect to the unknowns are evaluated analytically for the SRK equation of state, whereas the derivatives of $K_{i}^{a}$ are determined numerically by means of a finite-difference method, see Appendix A.4.

\subsubsection{Initialization}

Convergence of the Newton-Raphson method relies on whether the initial estimate lies within the domain of attraction. Several initialization methods have been proposed in the literature, e.g. Ref. [36], which are based on low-pressure relations for $K_{i}^{\alpha}$. For high pressures this zeroth order scheme may be inadequate and therefore we develop an initialization scheme based on first order pressure extrapolation of the solution variables $\boldsymbol{\xi}$. We write Eq. (2.17) as

$$
\mathbf{G}(p, T)=\mathbf{F}(\boldsymbol{\xi}(p, T) ; p, T)=\mathbf{0}, \quad \forall p, T .
$$

The extrapolation uses the derivative of $\boldsymbol{\xi}$ with respect to the pressure, which can be extracted from

$$
\frac{\partial \mathbf{G}}{\partial p}=\mathrm{J} \frac{\partial \boldsymbol{\xi}}{\partial p}+\frac{\partial \mathbf{F}}{\partial p}=\mathbf{0} .
$$

The first-order extrapolation of $\boldsymbol{\xi}(p+\Delta p, T)$ is then

$$
\boldsymbol{\xi}(p+\Delta p, T)=\boldsymbol{\xi}(p, T)-\mathrm{J}^{-1} \frac{\partial \mathbf{F}}{\partial p} \Delta p+O\left(\Delta p^{2}\right) .
$$

The derivative of $\mathbf{F}$ with respect to the pressure only affects the first $2 N$ equations of Eq. (2.17) containing $K_{i}^{\alpha}$ and is given by

$$
\frac{\partial \mathbf{F}}{\partial p}=\left(-x_{1}^{c} \frac{\partial K_{1}^{c}}{\partial p}, \ldots,-x_{N}^{c} \frac{\partial K_{N}^{c}}{\partial p},-x_{1}^{a} \frac{\partial K_{1}^{a}}{\partial p}, \ldots,-x_{N}^{a} \frac{\partial K_{N}^{a}}{\partial p}, \mathbf{0}\right)^{\mathrm{T}} .
$$

A similar strategy can be used for extrapolation of $\boldsymbol{\xi}(p, T)$ in terms of the temperature.

\subsection{Results}

Flash calculations have been performed using the Soave-Redlich-Kwong equation of state for a mixture of ethane $(k=1)$, n-nonane $(k=2)$ and water $(k=3)$. The feed fractions are $\mathbf{z}=(0.5,0.1,0.4)$. Fig. 2.1 shows the three-phase envelope for the ternary mixture in the pressure-temperature domain. The domain surrounding the three-phase region consists either of the single phase vapor (lower right domain) or the two-phase liquid (upper left domain). 
The condensate and aqueous phase fractions, denoted by $C$ and $\mathcal{A}$, respectively, are depicted in Fig. 2.1(a)-(b). The phase transition from the vapor to the three-phase region by means of isobaric cooling results in a liquid which mainly contains aqueous components. Further decreasing the temperature leads to a constant value of $\mathcal{A}$ and increasing condensate fraction $C$.

The vapor fractions of $n$-nonane and water are given in Fig. 2.1(c)-(d). They can be considered as the 'heavy' components in the mixture and therefore the vapor fractions decrease rapidly as the temperature is decreased. Near the liquid phase region the vapor consists mainly of ethane.

\subsection{Conclusions}

In this chapter a method has been presented for solving the complete system of equations for equilibrium calculations. The equilibrium variables are part of the solution vector and are not subject to additional constraints. For the Newton-Raphson method to converge, it is essential to provide an adequate initialization method. For that we propose a pressure extrapolation scheme. Initialization schemes based on lowpressure expressions [36] are insufficiently accurate and can lead to incorrect results. The derivatives needed in the iterative scheme and the initialization scheme are calculated analytically for the hydrocarbon components which are accurately described by the SRK equation of state. 


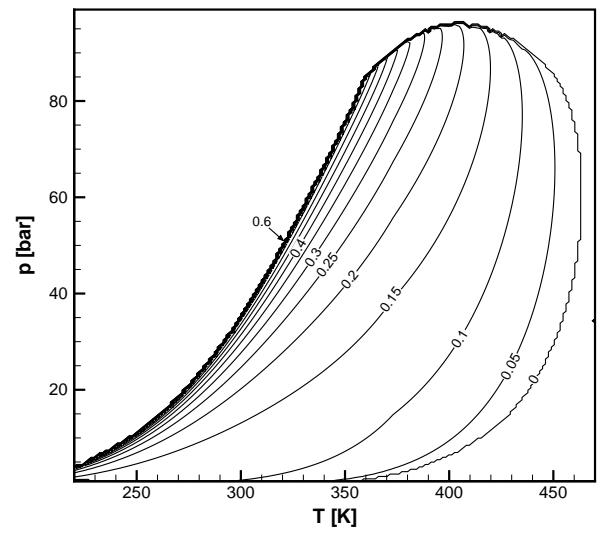

(a) $C$, condensate fraction

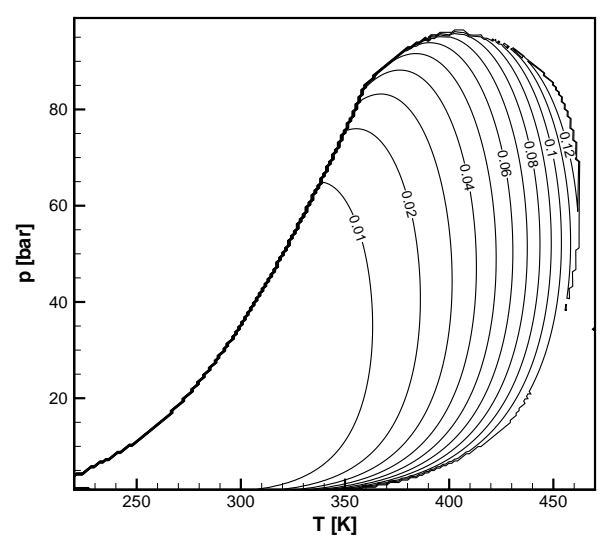

(c) $x_{2}^{v}$, n-nonane vapor fraction

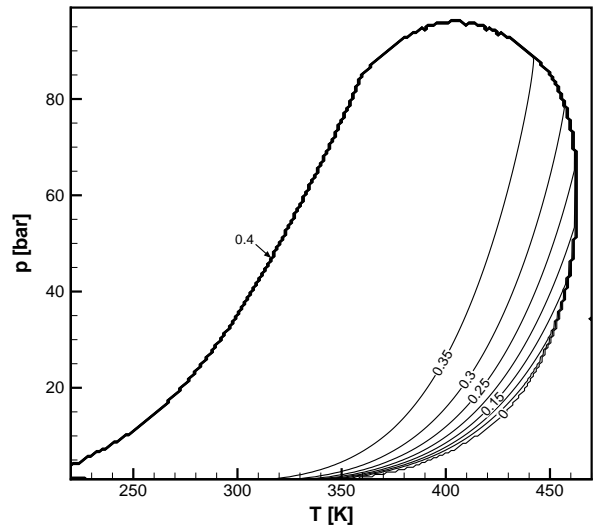

(b) $\mathcal{A}$, aqueous fraction

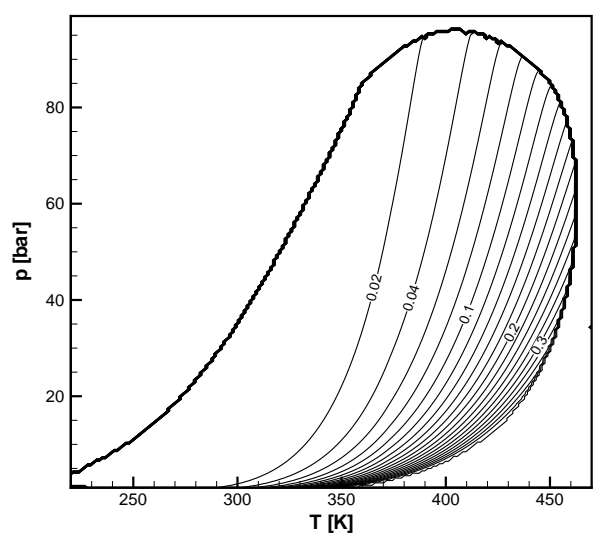

(d) $x_{3}^{v}$, water vapor fraction

Figure 2.1: Phase diagrams for mixture of ethane $(k=1), n$-nonane $(k=2)$ and water $(k=3)$ with feed mole fractions $\mathbf{z}=(0.5,0.1,0.4)$ based on the SRK equation of state. Iso-contours of: (a) condensate liquid fraction $C$, (b) aqueous liquid fraction $\mathcal{A},(c)$ vapor fraction of n-nonane $x_{2}^{v}$ and $(d)$ vapor fraction of water $x_{3}^{v}$. 


\section{MODELS FOR $N$-COMPONENT CONDENSATION}

The various physical models that are used to describe $N$-component condensation are reviewed. All models presented aim at evaluating the cluster size distribution but differ in their accuracy and complexity. We start with the computationally most challenging model: the N-component Becker-Döring (NBD) equations which are most complete in their physical description and can be considered as the 'exact' model. Then, the N-component Fokker-Planck Equation is derived by continuation of the $N B D$ equations. Further simplification of this equation leads to the $N$-component General Dynamic Equation.

\subsection{Becker-Döring (NBD) equations}

The kinetic process of condensation is described by the $N$-component Becker-Döring (NBD) equations [4, 68]. The cluster evolution is considered as a sequence of elementary processes of attachment and detachment of monomers. The NBD equations describe the time rate of change of the $N$-component $\mathbf{n}$-cluster number density, $\rho_{\mathbf{n}}$, due to the fluxes towards and from neighboring clusters:

$$
\frac{d \rho_{\mathbf{n}}}{d t}=\sum_{k=1}^{N}\left\{J_{\mathbf{n}-\mathbf{e}_{k}}^{k}-J_{\mathbf{n}}^{k}\right\}
$$

where $\mathbf{n}=\left(n_{1}, n_{2}, \ldots, n_{N}\right)^{\mathrm{T}} \in \mathbb{N}^{N}$ and $\mathbf{e}_{k}$ is the $k^{\text {th }}$ unity vector. The flux at $\mathbf{n}$ in $k$-direction, $J_{\mathbf{n}}^{k}$, is constructed by considering the forward rate, $f_{\mathbf{n}}^{k}$, and the backward rate, $b_{\mathbf{n}}^{k}$, as

$$
J_{\mathbf{n}}^{k}=f_{\mathbf{n}}^{k} \rho_{\mathbf{n}}-b_{\mathbf{n}+\mathbf{e}_{k}}^{k} \rho_{\mathbf{n}+\mathbf{e}_{k}} .
$$

In the model represented by Eqs. (3.1) and (3.2) it is assumed that the $\mathbf{n}$-clusters can change in size due to addition or extraction of a single $k$-component monomer only, which is justified due to the fast decreasing number density with increasing size governed by the Boltzmann size distribution, see Eq. (3.7). A more general description taking into account the kinetics between all cluster sizes is given in Ref. [23]. The Becker-Döring process is illustrated in Fig. 3.1 for the binary case, i.e. $N=2$. 


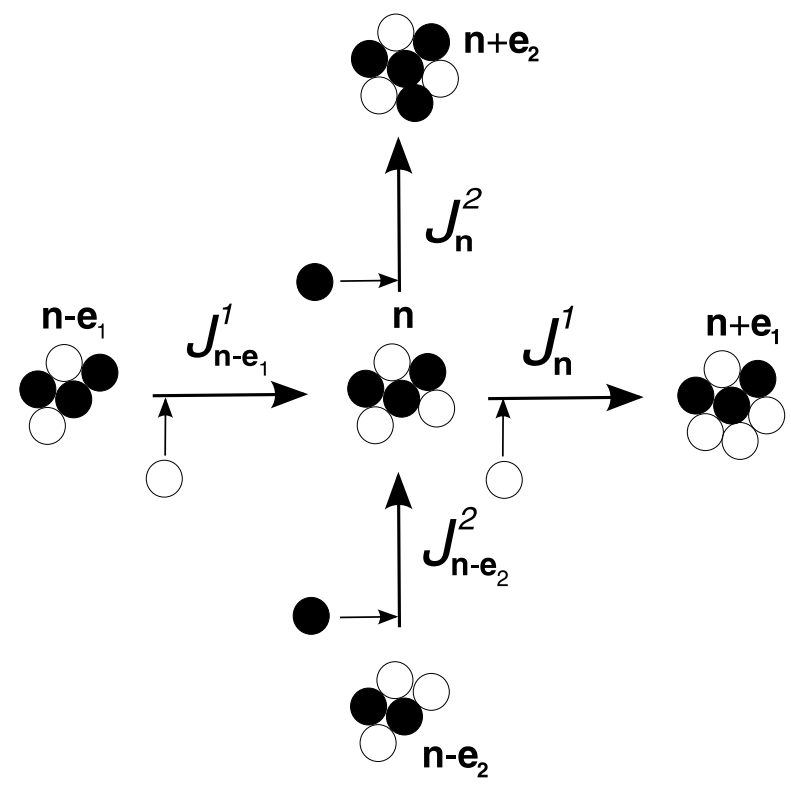

Figure 3.1: Kinetic process described by the Becker-Döring equations for $N=2$ at the $\mathbf{n}$-cluster. The open and solid circles correspond to component 1 and component 2 monomers, respectively.

The forward rate is determined by the impingement rate of vapor molecules on the existing $\mathbf{n}$-cluster and is derived from gas kinetics as [14, 32]

$$
f_{\mathbf{n}}^{k}=\rho_{\mathbf{e}_{k}}\left(s_{\mathbf{e}_{k}}^{\frac{1}{2}}+s_{\mathbf{n}}^{\frac{1}{2}}\right)^{2} \sqrt{\frac{k_{\mathrm{B}} T\left(m_{\mathbf{e}_{k}}+m_{\mathbf{n}}\right)}{2 \pi m_{\mathbf{e}_{k}} m_{\mathbf{n}}}},
$$

with $\rho_{\mathbf{e}_{k}}=y_{k} p / k_{\mathrm{B}} T$ the monomer number density of component $k$ and $m_{\mathbf{e}_{k}}$ its molecular mass. Furthermore, $s_{\mathbf{n}}$ and $m_{\mathbf{n}}$ are the surface area and the mass of the $\mathbf{n}$-cluster, respectively, given by

$$
s_{\mathbf{n}}=(36 \pi)^{\frac{1}{3}} v_{l, \mathbf{n}}^{\frac{2}{3}}, \quad \text { with } \quad v_{l, \mathbf{n}}=\sum_{k=1}^{N} n_{k} v_{l, \mathbf{e}_{k}},
$$

where $v_{l, \mathbf{e}_{k}}$ is the partial molecular volume of component $k$ and

$$
m_{\mathbf{n}}=\sum_{k=1}^{N} n_{k} m_{\mathbf{e}_{k}} .
$$

The backward rate is determined by the detailed balance condition at constrained equilibrium, implying that the equilibrium size distribution satisfies Eq. (3.2) for $J_{\mathbf{n}}^{k}=$ 
$0, \forall \mathbf{n}, k$ yielding

$$
b_{\mathbf{n}}^{k}=f_{\mathbf{n}-\mathbf{e}_{k}}^{k} \frac{\rho_{\mathbf{n}-\mathbf{e}_{k}}^{\mathrm{eq}}}{\rho_{\mathbf{n}}^{\mathrm{eq}}},
$$

where $\rho_{\mathbf{n}}^{\mathrm{eq}}$ is the Boltzmann equilibrium size distribution for $N$ components [32]

$$
\rho_{\mathbf{n}}^{\mathrm{eq}}=\rho_{\mathbf{n}}^{0} \exp \left(-g_{\mathbf{n}}\right), \quad \text { with } \quad g_{\mathbf{n}} \equiv \frac{\Delta G_{\mathbf{n}}}{k_{\mathrm{B}} T},
$$

and where $\Delta G_{\mathbf{n}}$ is the Gibbs free energy of cluster formation, $k_{\mathrm{B}}$ is the Boltzmann constant, $T$ is the temperature and $\rho_{\mathbf{n}}^{0}$ is the normalization constant. The relation for the Gibbs free energy is discussed in Section 3.1.1.

The NBD problem can be solved for given boundary conditions at the monomer number densities, i.e. at $\rho_{\mathbf{e}_{k}}$. A thorough elaboration of Penrose [3,38] proves that given the correct initial and boundary conditions the solution of the BD equations exists and is unique.

For realistic condensation problems, in which clusters reach a size up to $10^{7}$ monomers, the system of equations (3.1) leads to a numerical task for an $N$-component mixture that cannot be carried out due to the required large memory storage and computational effort. Therefore, computations based on solving the NBD-equations either use a limited region in $\mathbf{n}$-space $[47,81]$ or use a sectional method in which ranges of cluster sizes are grouped [65].

\subsubsection{Equilibrium cluster size distribution}

The NBD equations and the deduced models require the equilibrium distribution (3.7) containing the Gibbs free energy of formation of an $\mathbf{n}$-cluster. In the proceeding part of the thesis we will mainly consider the vapor-liquid transition of alcohol mixtures and apply the energy of formation used by Refs. [78, 81] and extend it to $\mathrm{N}$ components. The Gibbs free energy comprises a negative bulk term (which is the potential to form the liquid phase) and the positive surface term given by

$$
g_{\mathbf{n}}=-\sum_{k=1}^{N} n_{k} \ln \left(\frac{a_{k}}{x_{k}}\right)+\frac{\sigma_{\mathbf{n}} s_{\mathbf{n}}}{k_{\mathrm{B}} T},
$$

where $a_{k}=y_{k} p / p_{k}^{s}$ is the vapor phase activity with $y_{k}$ and $p_{k}^{s}$ the vapor mole fraction and the saturation pressure of the $k$-component, respectively. The vapor phase activity can be considered as the $N$-component equivalent of the supersaturation. In Eq. (3.8), $x_{k}=n_{k} / \sum_{l=1}^{N} n_{l}$ is the liquid fraction and $\sigma_{\mathbf{n}}$ is the surface tension of the $\mathbf{n}$-cluster. The latter is often assumed equal to the plain layer surface tension (with composition corresponding to the $\mathbf{n}$-cluster) which is termed the capillarity approximation. The activity coefficients of the liquid phase are assumed unity due to the ideality of the alcohol mixture [71, 78]. Moreover, the clusters are assumed well-mixed meaning 
that the possibility of surface enrichment of one of the constituents is not accounted for. The $\sigma_{\mathbf{n}}$ in Eq. (3.8) is given by [31]

$$
\sigma_{\mathbf{n}}=\sum_{k=1}^{N} x_{k} \sigma_{k},
$$

where $\sigma_{k}$ is the surface tension of the pure $k$-component.

The critical cluster size, $\mathbf{n}^{*}$, is defined as the saddle point of the function $g(\mathbf{n})$, which represents the lowest energy barrier for growing clusters [21, 49]. To form stable liquid clusters the flux from the $k$-component monomer densities needs to pass this energy barrier. Therefore, the main flux of clusters is expected to pass through $\mathbf{n}^{*}$. In Fig. 3.2 the shape of the Gibbs free energy of a binary mixture is illustrated. Three possible nucleation paths are drawn of which the one passing through the critical size $\mathbf{n}^{*}$ is energetically the most favorable.

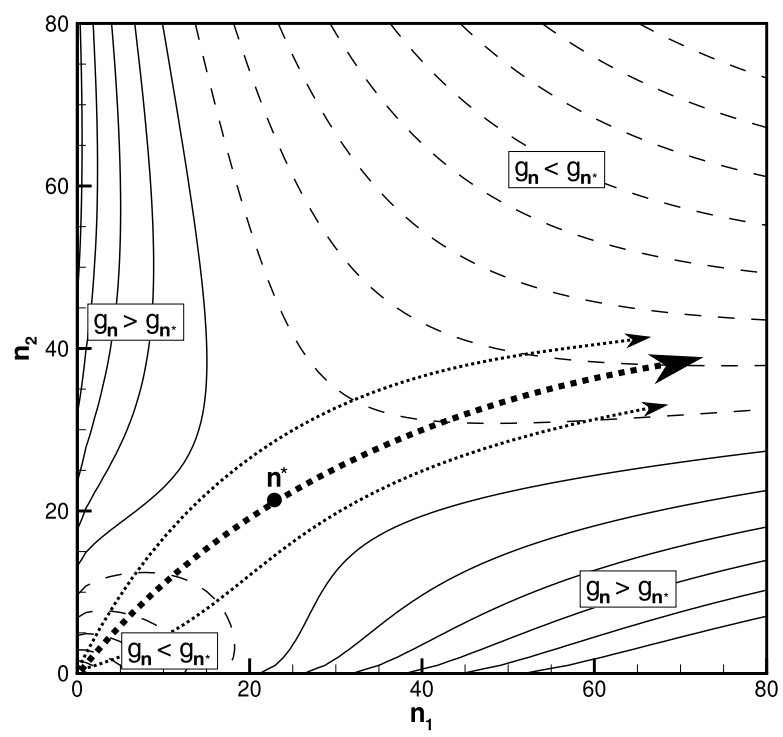

FIGURE 3.2: Iso-contours of the Gibbs free energy of formation for the binary ethanolhexanol mixture in $\left(n_{1}, n_{2}\right)$-space, i.e. $N=2$. Solid iso-lines correspond to $g_{\mathbf{n}}>g_{\mathbf{n}^{*}}$, dashed iso-lines correspond to $g_{\mathbf{n}}<g_{\mathbf{n}^{*}}$. The dot corresponds to the saddle point of $g_{\mathbf{n}}$ referred to as the critical size $\mathbf{n}^{*}$. The nucleation paths are illustrated by the dotted lines with the thick dotted line corresponding to the main flux path passing through $\mathbf{n}^{*}$.

The normalization constant, $\rho_{\mathbf{n}}^{0}$, in Eq. (3.7) has been subject to discussion in literature, see e.g. Ref. [78]. In its original form, devised by Reiss [49], the constant is given by the sum of the monomer number densities of all components, i.e. 
$\rho^{0}=\sum_{k=1}^{N} \rho_{\mathbf{e}_{k}}$ and is not a function of the cluster composition. Using this normalization constant in Eq. (3.7) leads to unphysical limits of the equilibrium size distribution. These limits comprise the reduction to the unary equilibrium distribution and the limiting behavior near the monomer densities. The normalization derived by Ref. [78] ensures that the correct limits are obeyed and is termed self-consistent. The normalization 'constant', $\rho_{\mathbf{n}}^{0}$ depends on the cluster composition and reads for $N$-components:

$$
\rho_{\mathbf{n}}^{0}=\prod_{k=1}^{N}\left[\rho_{\mathbf{e}_{k}}^{s} \exp \left(\theta_{k}\right)\right]^{x_{k}}
$$

where $\rho_{\mathbf{e}_{k}}^{s}$ is the saturated monomer number density of component $k$ and $\theta_{k} \equiv s_{\mathbf{e}_{k}} \sigma_{k} / k_{\mathrm{B}} T$.

Although the self-consistent form satisfies the required limits for the $N$-component equilibrium size distribution, the model has several drawbacks. The surface contribution in $g(\mathbf{n})$ is based on the capillarity approximation which loses its meaning for small clusters. Also, the equilibrium size distribution cannot be applied to supercritical fluids since $\sigma_{k}$ and $\rho_{\mathbf{e}_{k}}^{s}$ are indeterminate. Recent studies [24, 25, 34] attempted to improve the form of $g(\mathbf{n})$ and $\rho_{\mathbf{n}}^{0}$ based on a statistical mechanical approach for $N=1,2$. However, a general expression for $N$-component mixtures is not yet obtained.

\subsection{Fokker-Planck Equation (NFPE)}

An alternative for the NBD equations is the $N$-component Fokker-Planck Equation (NFPE) which can be derived by continuation of $\rho(\mathbf{n}, t)$ to non-integer values of $\mathbf{n}$. A Taylor series expansion of the right-hand side (rhs) of system $(3.1)$ [66, 85] leads to

$$
\frac{\partial \rho(\mathbf{n}, t)}{\partial t}=\sum_{k=1}^{N} T_{L}^{k}(\rho, \mathbf{n}, t)
$$

with

$$
T_{L}^{k}(\rho, \mathbf{n}, t) \equiv \sum_{l=1}^{L} \frac{1}{l !} \frac{\partial^{l}}{\partial n_{k}^{l}}\left[\left((-1)^{l} f^{k}(\mathbf{n})+b^{k}(\mathbf{n})\right) \rho(\mathbf{n}, t)\right] .
$$

Eq. (3.11) is referred to as the generalized NFPE [50] for $L \rightarrow \infty$. The rhs terms become smaller as the order increases [66], therefore to good approximation we can truncate the summation in Eq. (3.12) at the second-order term yielding the NFPE

$$
\frac{\partial \rho}{\partial t} \approx-\sum_{k=1}^{N}\left[\frac{\partial}{\partial n_{k}}\left\{\left(f^{k}(\mathbf{n})-b^{k}(\mathbf{n})\right) \rho\right\}-\frac{\partial^{2}}{\partial n_{k}^{2}}\left\{\frac{1}{2}\left(f^{k}(\mathbf{n})+b^{k}(\mathbf{n})\right) \rho\right\}\right] .
$$

The first term in the rhs is the drift term and the second term is the diffusion term containing the diffusion coefficient $d_{k}(\mathbf{n}) \equiv \frac{1}{2}\left(f^{k}(\mathbf{n})+b^{k}(\mathbf{n})\right)$. The error introduced 
by neglecting the higher-order terms can give unphysical results for $\rho(\mathbf{n}, t)$ in the sense that the equilibrium distribution is not a solution of Eq. (3.13) as demonstrated in Ref. [66].

We can circumvent this problem by using a more convenient form of Eq. (3.13) $[67,85]$ :

$$
\frac{\partial \rho}{\partial t}=-\sum_{k=1}^{N}\left[\frac{\partial}{\partial n_{k}}\left(\dot{n}_{k} \rho-d_{k} \frac{\partial \rho}{\partial n_{k}}\right)\right],
$$

where the term between the round brackets is the nucleation flux $J^{k}(\mathbf{n})=\dot{n}_{k} \rho-d_{k} \frac{\partial \rho}{\partial n_{k}}$ with growth rate $\dot{n}_{k}$. Eq. (3.14) can be recast in vector form as

$$
\frac{\partial \rho}{\partial t}+\nabla_{\mathbf{n}} \cdot(\dot{\mathbf{n}} \rho)=\nabla_{\mathbf{n}} \cdot\left(\mathrm{D} \nabla_{\mathbf{n}} \rho\right)
$$

where $\nabla_{\mathbf{n}}=\left(\frac{\partial}{\partial n_{1}}, \frac{\partial}{\partial n_{2}}, \ldots, \frac{\partial}{\partial n_{N}}\right)^{\mathrm{T}}, \dot{\mathbf{n}}$ is the growth rate vector and $\mathrm{D}$ is the diagonal diffusion tensor with entries $d_{k}$.

The growth rate vector, $\mathbf{n}$, is chosen such that the $N$-component generalization of the fundamental Zeldovich relation [85] is satisfied:

$$
\dot{\mathbf{n}}(\mathbf{n})=\mathrm{D}(\mathbf{n}) \nabla_{\mathbf{n}}\left[\ln \rho^{\mathrm{eq}}(\mathbf{n})\right],
$$

which ensures the correct Boltzmann equilibrium limit of Eq. (3.15): $J^{k}(\mathbf{n})=0, \forall \mathbf{n}, k$, when $\rho(\mathbf{n})=\rho^{\mathrm{eq}}(\mathbf{n})$. If the normalization constant $\rho^{0}(\mathbf{n})$ in the continuous form of Eq. (3.7) is not a function of cluster size, Eq. (3.16) reduces to $\dot{\mathbf{n}}(\mathbf{n})=-\mathrm{D}(\mathbf{n}) \nabla_{\mathbf{n}} g(\mathbf{n})$. Since the Gibbs free energy of formation exhibits a saddle point in $\mathbf{n}$-space the growth rate is zero at $\mathbf{n}^{*}$ and the drift flux $\dot{\mathbf{n}} \rho$ vanishes. As soon as clusters become sufficiently larger than $\mathbf{n}^{*}$ the drift flux becomes dominant and the diffusion flux can be neglected [46]. The diffusion term in Eq. (3.14) ensures that small clusters can become supercritical and grow to larger sizes. This behavior is further explained in Chapter 6.

The NFPE requires a numerical solution scheme that can handle the region-dependent hyperbolic/parabolic character of Eq. (3.15). Furthermore, for $N$-component mixtures we still need a large $\mathrm{N}$-dimensional computational grid and higher order numerical schemes are mandatory in order to control numerical diffusion.

\subsection{General Dynamic Equation (NGDE)}

We define the supercritical cluster size domain $\Omega^{*}$ in $\mathbf{n}$-space as the region in which $g(\mathbf{n})$ decreases if $\|\mathbf{n}\|$ increases. Noting that: $\frac{1}{2} \nabla_{\mathbf{n}}\|\mathbf{n}\|^{2}=\mathbf{n}$, we obtain the definition

$$
\Omega^{*} \equiv\left\{\mathbf{n} \in \mathbb{R}^{N} \mid \mathbf{n} \cdot \nabla_{\mathbf{n}} g(\mathbf{n})<0\right\}
$$


Instead of calculating the diffusion flux in the NFPE we can introduce clusters in the supercritical domain $\Omega^{*}$ at a source location $\mathbf{n}_{0}$ at a rate equal to the steady state nucleation rate $J_{s}$. This leads to the $N$-component General Dynamic Equation

$$
\frac{\partial \rho}{\partial t}+\nabla_{\mathbf{n}} \cdot(\dot{\mathbf{n}} \rho)=J_{\mathrm{s}} \delta\left(\mathbf{n}-\mathbf{n}_{0}\right),
$$

where the Dirac $\delta$-function introduces the clusters at $\mathbf{n}_{0}$ in $N$-dimensional cluster space. The NGDE allows for a very efficient solution strategy based on the hyperbolic character of Eq. (3.18). The steady state nucleation rate, $J_{\mathrm{s}}$, is obtained by solving the NBD equations (3.2) with $\frac{\partial \rho_{\mathbf{n}}}{\partial t}=0$, which is described in full detail in Chapter 6 . The implication of neglecting the diffusion term is that Eq. (3.18) is only applicable in the supercritical domain $\Omega^{*}$.

In its original form introduced in Ref. [52] for single component condensation, the source term of Eq. (3.18) is located at the critical cluster size, i.e. $\mathbf{n}_{0}=\mathbf{n}^{*}$. At fixed external conditions, however, the newly born critical clusters are in unstable equilibrium and therefore do not grow. This results in a cluster size distribution that is singular at $\mathbf{n}^{*}$. Moreover, a rapid change in external conditions, resulting in an increase of the critical size, leads to evaporation of all clusters. Recent studies made an attempt to overcome these deficiencies by replacing the $\delta$-function in Eq. (3.18) by a boundary condition at a size larger than the critical size $[22,66]$. We will extensively address the location of the source point $\mathbf{n}_{0}$ in Chapter 6. 



\section{A MULTIGRID METHOD FOR THE NBD EQUATIONS}

In this chapter a multigrid algorithm is developed enabling faster solution of the $\mathrm{N}$ component Becker-Döring equations for the cluster size distribution in $N$-component nucleation. The numerical method is elaborated for an arbitrary number of condensing components, making the simulation of many-component nucleating systems feasible. The method is applied to a steady state ternary nucleation problem in order to demonstrate its efficiency. The results are used as a validation for existing ternary nucleation theories. The method is also applied to a non-steady state ternary problem, which provides useful insight into the initial stages of the nucleation process.

The work in this chapter has been published in revised form as: D.S. van Putten, S.P. Glazenborg, R. Hagmeijer and C.H. Venner, J. Chem. Phys. 135, 014114 (2011).

\subsection{Introduction}

The nucleation rate of $N$-component mixtures is an important parameter in many fields of physics, e.g. vapor-liquid transition [73, 83], crystallization [51], atmospheric aerosols $[15,77]$ and metallurgy science [48]. To calculate the steady state nucleation rate, the $N$-component generalization $[21,74]$ of the classical binary nucleation theory of Reiss [49] is often used. The $N$-component nucleation theory applies assumptions in the saddle point region of the Gibbs free energy in order to find an analytic solution of the nucleation rate. This theory provides steady state nucleation rates only.

To circumvent the assumptions of the classical theory, the full $N$-component BeckerDöring (NBD) equations [4] need to be solved. Many studies concern the numerical solution of the binary Becker-Döring equations $[81,35]$ and to obtain the steady state size distribution requires long simulation times [46]. Moreover, increasing the number of nucleating components leads to an exponential increase of the computational effort and results in a practically unsurmountable numerical task. 
In the present chapter we propose a multigrid algorithm [6] for the calculation of the $N$-component size distribution. Multigrid methods have been applied successfully to various physical problems and show a tremendous gain in the efficiency for numerical methods for solving discretized partial differential equations $[7,76]$. However, these methods have been developed to suit a specific physical problem and generally will not succeed when applied to other systems. Therefore, we construct a geometric multigrid algorithm which is tailored to the NBD equations. The efficiency increase is demonstrated by means of solving a steady state ternary nucleation problem. The time evolution of the ternary cluster size distribution is calculated in the initial stages of the nucleation process providing useful insight on relaxation times and the time dependence of the main nucleation path.

The obtained method makes calculations of $N$-component nucleation feasible and can be used to validate the assumptions of $N$-component nucleation theories. Furthermore, the solution of reduced models of the NBD equations like the Fokker-Planck Equation [54], General Dynamic Equation [46] and Stationary Diffusion Flux equation [45] can be compared with the solution of the full NBD equations. Moreover, the method allows for more complicated relations for the Gibbs free energy, e.g. Ref. [25, 84].

\section{2 $N$-component Becker-Döring equations}

The NBD equations describe the time rate of change of the $N$-component $\mathbf{n}$-cluster number density, $\rho_{\mathbf{n}}(t)$, as described in Chapter 3. Using Eq. (3.2) in Eq. (3.1) yields the system of first-order ordinary differential equations

$$
\frac{d \rho_{\mathbf{n}}}{d t}=\sum_{k=1}^{N}\left\{f_{\mathbf{n}-\mathbf{e}_{k}}^{k} \rho_{\mathbf{n}-\mathbf{e}_{k}}-\left(f_{\mathbf{n}}^{k}+b_{\mathbf{n}}^{k}\right) \rho_{\mathbf{n}}+b_{\mathbf{n}+\mathbf{e}_{k}}^{k} \rho_{\mathbf{n}+\mathbf{e}_{k}}\right\},
$$

where $\mathbf{n}=\left(n_{1}, n_{2}, \ldots, n_{N}\right)^{\mathrm{T}} \in \mathbb{N}^{N}$ and $\mathbf{e}_{k}$ is the $k^{\text {th }}$ unity vector. In the case of vaporliquid transition the forward rate is given by gas kinetics [14, 32] (see Section 3.1) and the backward rate is determined by the detailed balance condition at constrained equilibrium

$$
b_{\mathbf{n}}^{k}=f_{\mathbf{n}-\mathbf{e}_{k}}^{k} \frac{\rho_{\mathbf{n}-\mathbf{e}_{k}}^{\mathrm{eq}}}{\rho_{\mathbf{n}}^{\mathrm{eq}}},
$$

where $\rho_{\mathbf{n}}^{\mathrm{eq}}$ is the binary equilibrium size distribution

$$
\rho_{\mathbf{n}}^{\mathrm{eq}}=\rho_{\mathbf{n}}^{0} \exp \left(-g_{\mathbf{n}}\right), \quad \text { with } \quad g_{\mathbf{n}} \equiv \frac{\Delta G_{\mathbf{n}}}{k_{\mathrm{B}} T},
$$

where $\Delta G_{\mathbf{n}}$ is the Gibbs free energy of cluster formation, $k_{\mathrm{B}}$ is the Boltzmann constant, $T$ is the temperature and $\rho_{\mathbf{n}}^{0}$ is the normalization constant. In this study we apply the self-consistent equilibrium size distribution of Refs. [78, 81], see Section 3.1.1. 


\subsection{Numerical solution method}

The time step of an explicit algorithm for the NBD equations (4.1) is limited by the Courant-Friedrichs-Lewy (CFL) condition [9] leading to very small time steps, e.g. of the order of $10^{-10} s$ in Ref. [46]. Therefore, we develop an implicit solver relieving us from this restriction. The first-order backward Euler time discretization [70] of Eq. (4.1) reads

$$
-\Delta t \sum_{k=1}^{N} f_{\mathbf{n}-\mathbf{e}_{k}}^{k} \rho_{\mathbf{n}-\mathbf{e}_{k}}^{m}+c_{\mathbf{n}} \rho_{\mathbf{n}}^{m}-\Delta t \sum_{k=1}^{N} b_{\mathbf{n}+\mathbf{e}_{k}}^{k} \rho_{\mathbf{n}+\mathbf{e}_{k}}^{m}=\rho_{\mathbf{n}}^{m-1},
$$

where $\Delta t$ is the time step size and

$$
c_{\mathbf{n}} \equiv 1+\Delta t \sum_{k=1}^{N}\left\{f_{\mathbf{n}}^{k}+b_{\mathbf{n}}^{k}\right\} .
$$

The superscripts $m-1$ and $m$ in Eq. (4.4) refer to the previous and current time level, respectively. This system can be cast in the form

$$
L \mathbf{u}=\mathbf{g},
$$

where $\mathbf{g}$ is the known right hand side containing the $\rho_{\mathbf{n}}$ 's at time $m-1$ and $\mathbf{u}$ contains the $\rho_{\mathbf{n}}$ 's at the present time $m$.

The number density of the monomers, i.e. $\mathbf{n}=\mathbf{e}_{k}$, are assumed constant and given by the pure $k$-component vapor number densities, i.e.

$$
\rho_{\mathbf{e}_{k}}=\rho_{v, k}, \quad \text { for } \quad k=1,2, \ldots, N .
$$

The system is subject to the boundary conditions for the flux $J^{k}$ defined in Eq. (3.2)

$$
\begin{array}{cl}
J_{\mathbf{n}-\mathbf{e}_{k}}^{k}=0, & \text { if } \quad n_{k}=0, \\
J_{\mathbf{n}}^{k}=J_{\mathbf{n}-\mathbf{e}_{k}}^{k}, & \text { if } \quad n_{k}=n_{e, k},
\end{array}
$$

where $n_{e, k}$ is the maximum cluster size of component $k$ in the computational domain chosen such that it is located sufficiently far from the critical size $\mathbf{n}^{*}$. To increase the stability of the numerical scheme, a constant flux boundary condition is applied at $n_{k}=n_{e, k}[81]$.

To solve this system we use the Gauss-Seidel iterative procedure [70]. Let $L_{j} \mathbf{u}=g_{j}$ denote the $j^{\text {th }}$ equation of system (4.6) and $r_{j}=g_{j}-L_{j} \tilde{\mathbf{u}}$ denote the residual of the $j^{\text {th }}$ equation, where $\tilde{\mathbf{u}}$ is the current approximation of $\mathbf{u}$. The Gauss-Seidel relaxation scheme at a certain time level is given by

$$
\tilde{u}_{j}:=\tilde{u}_{j}+\left(\frac{\partial L_{j} \mathbf{u}}{\partial u_{j}}\right)^{-1} r_{j},
$$


It is commonly known that the convergence of this iterative procedure will slow down when the solution becomes smooth with respect to the grid size [6]. Therefore, we develop a geometric multigrid algorithm for solving the system (4.6).

\subsection{Multigrid}

Multigrid methods were first introduced for the fast solution of partial differential equations by Brandt [5]. Since then algorithms have been developed using the multigrid/multilevel methodology for many problems in mathematics, engineering and physics [7]. Multigrid algorithms obtain their efficiency reducing each error component in the numerical solution process at a scale at which this can be done most efficiently. Crucial to the method is detailed understanding of the behavior of the equations to be solved at different scales. To obtain an efficient algorithm requires: (i) adequate reduction of small scale errors using an iterative scheme, (ii) accurate representation of the fine scale behavior of remaining smooth components of the error on the coarser scales and (iii) suitable often problem dependent interscale corrections. A black-box approach is rarely successful.

An example of a flow diagram of a multigrid $V\left(v_{1}, v_{2}\right)$ algorithm is given in Fig. 4.1. Starting at a fine grid, the target grid at which the solution is required, a set of coarser grids referred to as levels are introduced. On each coarser grid specific error components are reduced by $v_{1}$ iterations on the way to a coarsest grid on which the final smoothest components are solved to machine precision. Subsequently all corrections are merged from coarse to fine grid, at each gridlevel carrying out an additional $v_{2}$ iterations.

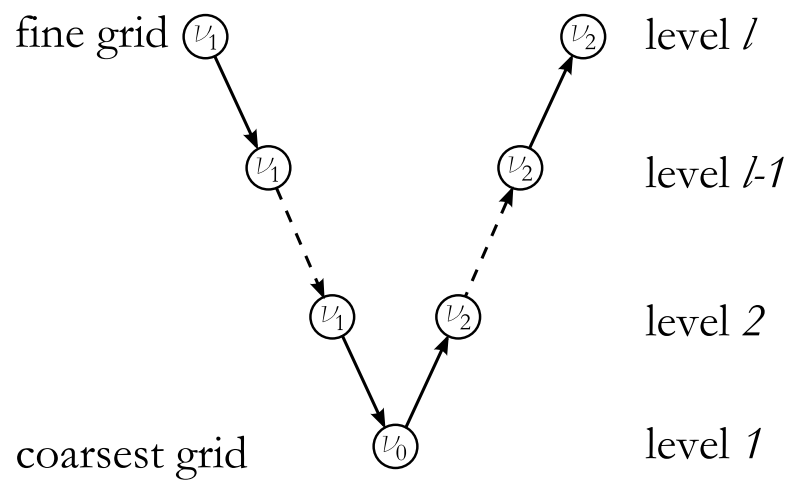

FIGURE 4.1: Representation of a multigrid $V$-cycle, $V\left(v_{1}, v_{2}\right)$, with $v_{1}$ pre-relaxations, $v_{2}$ post-relaxations and $v_{0}$ relaxations on the coarsest grid. 


\subsubsection{Coarse grid correction scheme}

The principle of coarse grid correction is described using 2 levels with a fine grid denoted by $h$ and a coarse grid denoted by $H$. We start with Eq. (4.6) on the fine grid, i.e. $L^{h} \mathbf{u}^{h}=\mathbf{g}^{h}$, and find the approximation $\tilde{\mathbf{u}}^{h}$ by means of $v_{1}$ relaxations. The residual $\mathbf{r}^{h}$ is defined as

$$
\mathbf{r}^{h}=\mathbf{g}^{h}-L^{h} \tilde{\mathbf{u}}^{h}
$$

which can be written as

$$
\mathbf{r}^{h}=L^{h} \mathbf{u}^{h}-L^{h} \tilde{\mathbf{u}}^{h}
$$

Defining the error on the fine grid as $\mathbf{v}^{h} \equiv \mathbf{u}^{h}-\tilde{\mathbf{u}}^{h}$, one obtains

$$
L^{h}\left(\tilde{\mathbf{u}}^{h}+\mathbf{v}^{h}\right)=L^{h} \tilde{\mathbf{u}}^{h}+\mathbf{r}^{h},
$$

which is the general form for non-linear operators referred to as the Full Approximation Scheme. In this scheme an approximation to the error $\mathbf{v}^{H}$ is solved from Eq. (4.13) represented at gridlevel $H$. However, the operator of the $N$-component Becker-Döring equations is linear. In that case it is more convenient to use the Correction Scheme. For a linear operator Eq. (4.13) reduces to

$$
L^{h} \mathbf{v}^{h}=\mathbf{r}^{h} .
$$

Due to the $v_{1}$ relaxations the resulting error $\mathbf{v}^{h}$ is smooth with respect to the fine grid and the relaxation process becomes less effective. We therefore transfer the error and residual to the coarse grid using the restriction operator, $I_{h}^{H}$, and solve

$$
L^{H} \mathbf{v}^{H}=I_{h}^{H} \mathbf{r}^{h} .
$$

After $v_{0}$ relaxations $\mathbf{v}^{H}$ is solved to machine precision and transferred by the interpolation operator $I_{H}^{h}$ to the fine grid in order to correct $\tilde{\mathbf{u}}^{h}$

$$
\tilde{\mathbf{u}}^{h}:=\tilde{\mathbf{u}}^{h}+I_{H}^{h} \mathbf{v}^{H}
$$

Finally, the errors introduced by interpolation of $\mathbf{v}^{H}$ on level $h$ are reduced by $v_{2}$ post-relaxations. If this scheme is used recursively on multiple levels the $V$-cycle is obtained.

For the multigrid scheme we need the restriction and interpolation operators, $I_{h}^{H}$ and $I_{H}^{h}$, respectively, and the coarse grid operator $L^{H}$. The success of the multigrid algorithm relies on the proper choice of these operators. 


\subsubsection{Geometrical coarsening}

We define the fine grid consisting of hypercubes of unit length in $\mathbf{n}$-space with corresponding cell centers. These cell centers represent all possible cluster compositions given by the integer number of monomers. Fig. 4.2 shows the coarsened grid in two component $\left(n_{1}, n_{2}\right)$-space. The fine and coarse grid cell centers are indicated by the dots and the open squares, respectively. We emphasize that only the values at the fine grid cell centers correspond to physically realizable cluster sizes.

For convenience the index space $\mathbf{i}$ is defined which corresponds with the compo-

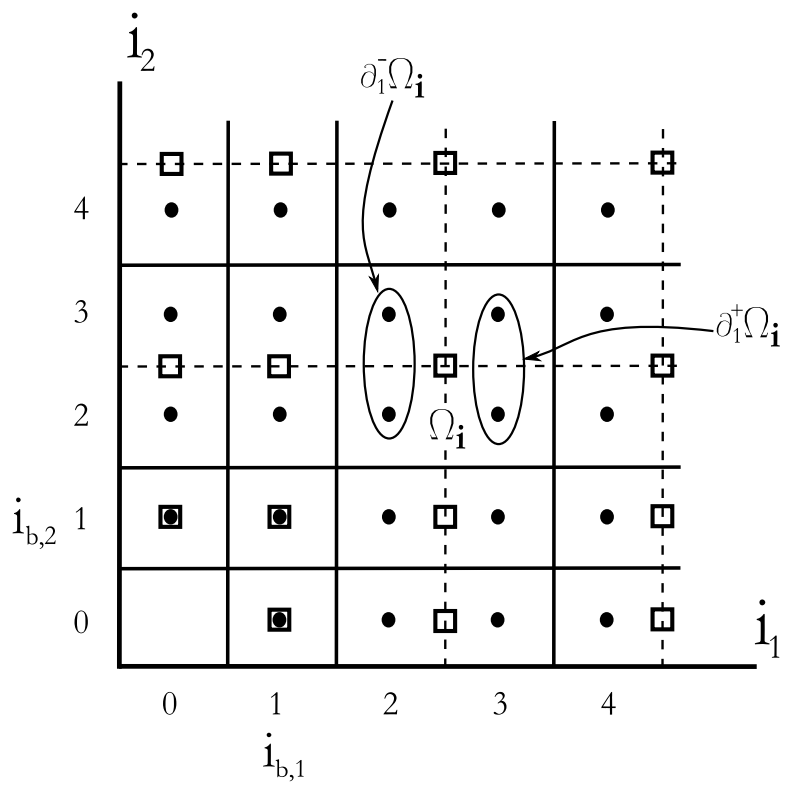

Figure 4.2: Coarse grid definition in two dimensional $\left(i_{1}, i_{2}\right)$-space. The solid and dashed lines are the cell boundaries on the fine grid and their cell centers are denoted by the dots. The solid lines are the cell boundaries on the coarse grid with cell centers indicated by the open squares. The set $\Omega_{\mathbf{i}}$ and its boundaries in $k=1$ direction, $\partial_{1}^{+} \Omega_{\mathbf{i}}$ and $\partial_{1}^{-} \Omega_{\mathbf{i}}$, are indicated.

sition space $\mathbf{n}$ on the fine grid, such that any variable $q$ defined in the composition $\mathbf{n}$-space is related to the $\mathbf{i}$-space by: $q_{\mathbf{i}}^{h} \equiv q_{\mathbf{n}}$.

On the coarse grid we define the index set in one component direction

$$
\Omega_{i}\left(i_{b}, i_{e}\right)= \begin{cases}\{i\}, & 0 \leq i \leq i_{b}, \\ \left\{2 i-i_{b}-1,2 i-i_{b}\right\}, & i_{b}<n \leq i_{e},\end{cases}
$$

where $i_{e}$ is the maximum cluster size and $i_{b}$ is the index below which the grid is not coarsened. This is done to accurately describe the expected steep gradients of 
the solution in the region of small clusters. The index set can be extended to higher dimensions

$$
\Omega_{\mathbf{i}}\left(\mathbf{i}_{b}, \mathbf{i}_{e}\right)=\left\{\mathbf{j} \in \mathbb{N}^{N} \mid j_{k} \in \Omega_{i_{k}}\left(i_{b, k}, i_{e, k}\right), 1 \leq k \leq N\right\} .
$$

Furthermore, let $\partial_{k}^{+} \Omega_{\mathbf{i}}$ and $\partial_{k}^{-} \Omega_{\mathbf{i}}$, as indicated in Fig. 4.2, denote the 'positive' and 'negative' boundaries of $\Omega_{\mathbf{i}}$ in direction $k$, respectively. These sets are defined as

$$
\partial_{k}^{ \pm} \Omega_{\mathbf{i}}=\left\{\mathbf{j} \in \mathbb{N}^{N} \mid \mathbf{j} \in \Omega_{\mathbf{i}}, j_{k} \in \partial^{ \pm} \Omega_{i_{k}}\right\} .
$$

with $\partial^{+} \Omega_{i}=\max \left(i, 2 i-i_{b}\right)$ and $\partial^{-} \Omega_{i}=\max \left(i, 2 i-i_{b}-1\right)$. With these definitions the restriction and interpolation operators are defined in the following section.

\subsubsection{Restriction and interpolation operators}

Let $G^{h}$ and $G^{H}$ denote the spaces of all grid functions on the fine and coarse grid, respectively. Then the restriction operator, $I_{h}^{H}$, and the corresponding interpolation $I_{H}^{h}$, are defined as

$$
\begin{aligned}
& I_{h}^{H}: G^{h} \mapsto G^{H}, g_{\mathbf{i}}^{H}=\frac{1}{\left|\Omega_{\mathbf{i}}\right|} \sum_{\mathbf{j} \in \Omega_{\mathbf{i}}} g_{\mathbf{j}}^{h}, \\
& I_{H}^{h}: G^{H} \mapsto G^{h}, \quad g_{\mathbf{j}}^{h}=g_{\mathbf{i}}^{H}, \forall \mathbf{j} \in \Omega_{\mathbf{i}} .
\end{aligned}
$$

We take $\rho^{H}=I_{h}^{H} \rho^{h}$. Similarly, the operator $L^{h}$ is restricted by means of Galerkin coarsening [6]

$$
L^{H}=I_{h}^{H} L^{h} I_{H}^{h}
$$

For convenience, the operators are considered further for the steady state of Eq. (4.1). The equation considered at point $\mathbf{i}$ of the steady state $L^{h} \mathbf{u}^{h}$ is given by

$$
-\sum_{k=1}^{N} f_{\mathbf{i}-\mathbf{e}_{k}}^{h,} \rho_{\mathbf{i}-\mathbf{e}_{k}}^{h}+c_{\mathbf{i}}^{h} \rho_{\mathbf{i}}^{h}-\sum_{k=1}^{N} b_{\mathbf{i}+\mathbf{e}_{k}}^{h, k} \rho_{\mathbf{i}+\mathbf{e}_{k}}^{h}=0 .
$$

Then $L^{H} \mathbf{u}^{H}$ can be written in a similar form as Eq. (4.23)

$$
-\sum_{k=1}^{N} f_{\mathbf{i}-\mathbf{e}_{k}}^{H, k} \rho_{\mathbf{i}-\mathbf{e}_{k}}^{H}+c_{\mathbf{i}}^{H} \rho_{\mathbf{i}}^{H}-\sum_{k=1}^{N} b_{\mathbf{i}+\mathbf{e}_{k}}^{H, k} \rho_{\mathbf{i}+\mathbf{e}_{k}}^{H}=0,
$$

where $c_{\mathbf{i}}^{H} \equiv \sum_{k=1}^{N}\left\{f_{\mathbf{i}}^{H, k}+b_{\mathbf{i}}^{H, k}\right\} . L^{H} \mathbf{u}^{H}$ contains the coarse grid forward rate, $f_{\mathbf{i}}^{H, k}$, and the coarse grid backward rate, $b_{\mathbf{i}}^{H, k}$, defined by

$$
f_{\mathbf{i}}^{H, k} \equiv \sum_{\mathbf{j} \in \partial_{k}^{+} \Omega_{\mathbf{i}}} f_{\mathbf{j}}^{h, k}, \quad \text { and } \quad b_{\mathbf{i}}^{H, k} \equiv \sum_{\mathbf{j} \in \partial_{k}^{-} \Omega_{\mathbf{i}}} b_{\mathbf{j}}^{h, k}
$$


This cell structured coarsening generates a coarse grid equation with the physical character of the fine grid equations for the smooth components that the coarse grid needs to correct. This is essential for good multigrid performance. It is emphasized that this favorable property is achieved when using the proposed cell coarsening and that other coarsening procedures may lead to non-converging coarse grid systems [16].

\subsection{Results and discussion}

We define a test case for the Ternary Becker-Döring (TBD) equations. The ideal alcohol mixture consists of ethanol $(k=1)$, propanol $(k=2)$, hexanol $(k=3)$ and an inert carrier gas (argon). The carrier gas does not influence the ternary nucleation process and serves as a heat sink in condensation experiments, see e.g. Ref. [71]. The vapor mole fractions of the condensing components are: $\mathbf{y}=\left(7.446 \cdot 10^{-3}, 1.818\right.$. $\left.10^{-3}, 1.026 \cdot 10^{-4}\right)$ and assumed non-depletable. The pressure $p$ and temperature $T$ are fixed such that a constant supersaturated state is attained, see Table 4.1. The alcohol mixture can be regarded as ideal and the pure properties are given in Appendix B.

\begin{tabular}{lc}
\hline Parameter & \\
\hline$p[\mathrm{kPa}]$ & 66.76 \\
$T[\mathrm{~K}]$ & 260.0 \\
$\mathbf{a}$ & $(1.0,1.2,4.0)$ \\
$\mathbf{n}^{*}$ & $(22,18,21)^{\mathrm{T}}$ \\
$g\left(\mathbf{n}^{*}\right)$ & 48.0 \\
\hline
\end{tabular}

TABLE 4.1: Test case conditions for the ternary ethanol, propanol and hexanol mixture with vapor composition $\mathbf{y}=\left(7.446 \cdot 10^{-3}, 1.818 \cdot 10^{-3}, 1.026 \cdot 10^{-4}\right)$.

\subsubsection{Steady state ternary nucleation}

The steady state solution of the TBD equations is obtained by solving Eq. (4.1) with $\frac{d \rho_{\mathbf{n}}}{d t}=0$. We demonstrate the efficiency increase accomplished by using the multigrid algorithm by comparing the convergence of the conventional Gauss-Seidel relaxation scheme against the $V(2,1)$-cycle multigrid algorithm. The three component computational domain consists of $128^{3}$ points. For convenient comparison we define a work unit (wu) as the amount of computational time needed for one fine grid relaxation. The $L_{2}$-norm of the residual (Eq. (4.11)) for $N$-components is defined as

$$
\|\mathbf{r}\|_{2}=\sqrt{\frac{1}{\prod_{k=1}^{N} i_{e, k}} \sum_{\mathbf{j} \in \Omega} r_{\mathbf{j}}^{2}},
$$


where $\Omega$ denotes the entire computational domain

$$
\Omega\left(\mathbf{i}_{e}\right)=\left\{\mathbf{i} \mid i_{k} \in\left\{0,1, \ldots, i_{e, k}\right\}, \forall k \in\{1,2, \ldots, N\}\right\} .
$$

For the test case we take $\mathbf{i}_{b}=\mathbf{n}^{*}$ since steep gradients in the solution are expected in the subcritical region, i.e. $\mathbf{i} \leq \mathbf{n}^{*}$. This ensures that the steep gradients are accurately described on all grid levels.

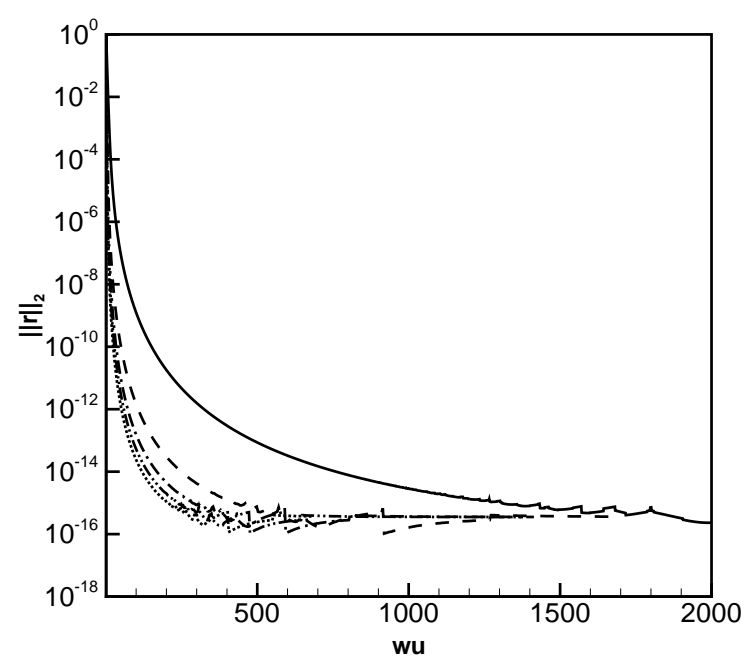

FiguRe 4.3: Convergence of residual for steady state ternary nucleation; GaussSeidel relaxation (solid) and V(2,1)-cycle with: $l=2$ (dashed), $l=3$ (dash-dot), $l=4$ (dash-dot-dot) and $l=5$ (dotted) on $128^{3}$ grid .

Fig. 4.3 shows the typical stalling convergence behavior for conventional GaussSeidel relaxation. Relaxation reduces the residuals effectively in the initial stage due to the reduction of high frequency error components. As these components are reduced the error becomes smoother and relaxation is unable to further reduce the error efficiently. The multigrid $V$-cycle algorithm continues the fast reduction of the error until the error becomes smooth on the coarsest grid. Therefore, the residuals of the multigrid algorithm with a larger number of levels continue the efficient error reduction. For $l=5$ an efficiency gain of approximately a factor 10 is achieved. It has to be noted that the relaxation process itself is a relatively effective solution method, when compared to the typical textbook examples [7], since the initial solution hardly contains low frequency error components. Therefore, the efficiency gain is not as high as reported in literature. 
The numerical solution of the steady state problem is used to calculate the steady state nucleation rate

$$
J_{\mathrm{s}} \equiv \sum_{n_{2}, n_{3}} J_{\mathbf{n}}^{1} .
$$

This numerical result is compared with the result from the analytic expression given by the classical nucleation theory, e.g. see Ref. [81]. For comparison we define the norm of the vapor activities, $|\mathbf{a}|$, as in Refs. $[71,78]$

$$
|\mathbf{a}|=\sqrt{a_{1}^{2}+a_{2}^{2}+a_{3}^{2}} .
$$

The ratios between the vapor activities are maintained and similar to the ones presented in Table 4.1, i.e. $a_{2} / a_{1}=1.2$ and $a_{3} / a_{1}=4$.

The steady state nucleation rates as a function of $|\mathbf{a}|$ are depicted in Fig. 4.4 for different temperatures. As will be shown in the next section the assumptions made to obtain an analytic expression, see e.g. Ref. [31, 81] and Section 6.2, are not always justified. However, the steady state nucleation rates agree within one order of magnitude with the numerically obtained values over a range of temperatures and $|\mathbf{a}|$.

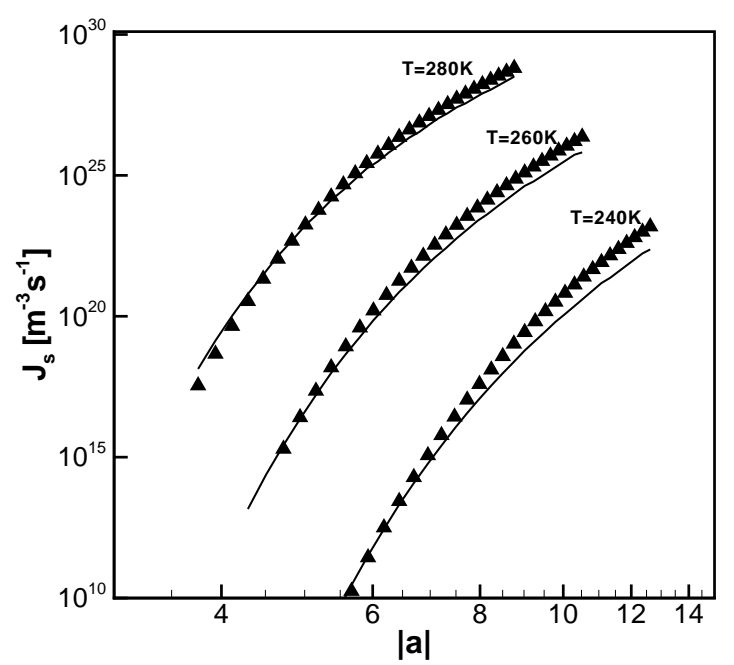

Figure 4.4: Comparison of theoretical and numerical steady state nucleation rates for $T=240 K, T=260 K$ and $T=280 K ; J_{s}$ from steady state nucleation theory (solid line) and from present numerical results (triangles). 


\subsubsection{Transient ternary nucleation}

We solve the TBD equations during the first $10 \mu \mathrm{s}$ of the formation process. The grid dimensions are $128^{3}$ and the time step is $5 \cdot 10^{-3} \mu s$. One single time step needs approximately 3 wu to converge to $\|\mathbf{r}\|_{2}=10^{-14}$ for the $V$-cycle (with $l=6$ ), whereas relaxation requires $18 \mathrm{wu}$. The efficiency increase obtained by using the multigrid technique is smaller compared to that of the steady state case. This can be explained by the presence of the time step in the operator $L$, increasing the diagonal dominance of $L$ with decreasing time step size. The increased diagonal dominance of the operator makes the relaxation process more efficient, therefore reducing the efficiency gain of the multigrid method. The convergence history of the transient case is plotted in Fig. 4.5.

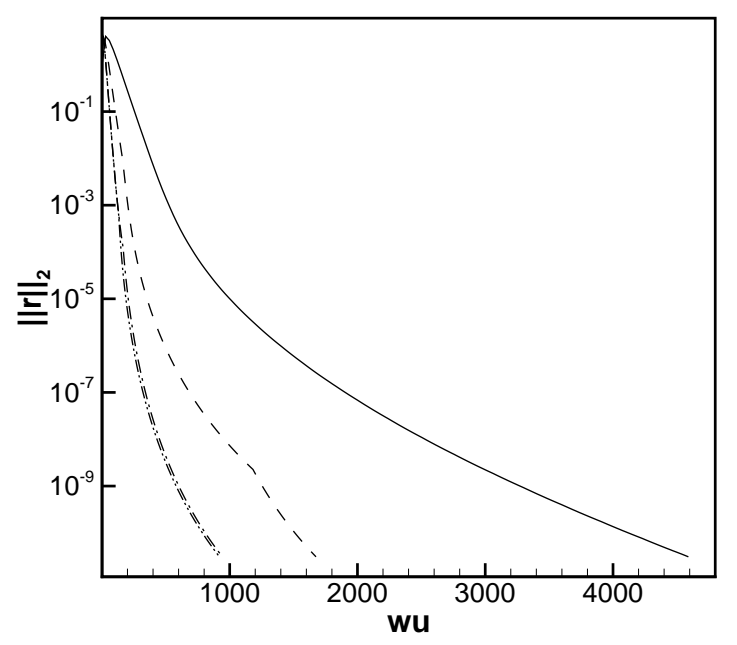

FIGURE 4.5: Convergence of time dependent nucleation; Gauss-Seidel relaxation (solid) and V(2,1)-cycle with: $l=2$ (dashed), $l=4$ (dash-dot) and $l=6$ (dashdot-dot) on $128^{3}$ grid. Residual is evaluated at new time level and is reduced to $10^{-14}$ for each time step.

The cluster size distribution can be plotted in the three component $\mathbf{n}$-space at several time instants, see Fig. 4.6. In the initial stage of the nucleation process (Fig. 4.6(a)) the distribution is transported mainly along the $n_{1}$-axis. This is due to the large vapor number density of ethanol which results in a higher forward rate and consequently a faster transport in $n_{1}$-direction.

This is confirmed by considering the main nucleation path in Fig. 4.7. The quan- 


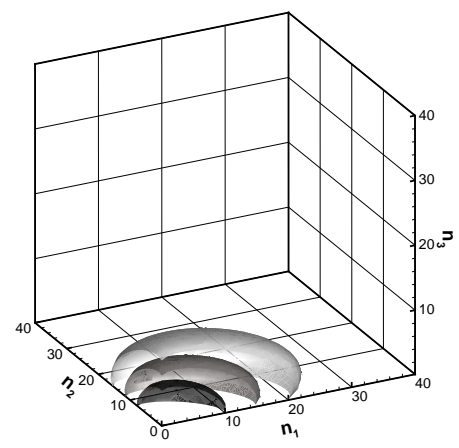

(a) $t_{1}=0.1 \mu \mathrm{s}$

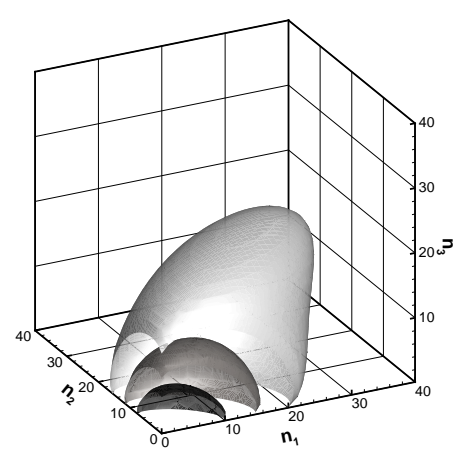

(c) $t_{1}=6 \mu \mathrm{s}$

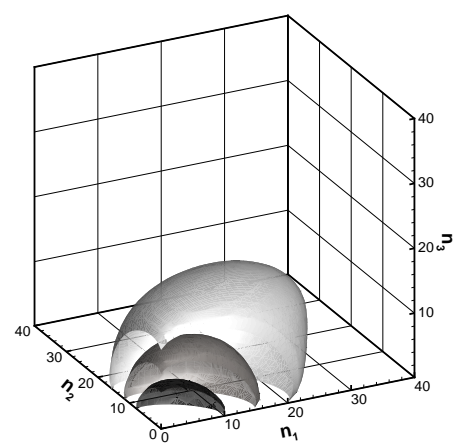

(b) $t_{1}=1 \mu \mathrm{s}$

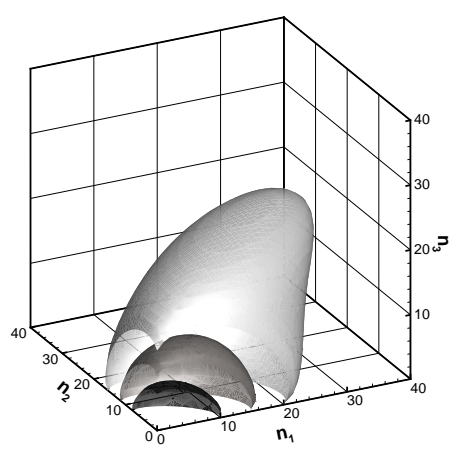

(d) $t_{1}=10 \mu \mathrm{s}$

Figure 4.6: Cluster size distribution, isosurface of $\log _{10} \rho=12$ (black), 8(dark grey) and 4 (light grey) at (a) $t_{1}=0.1 \mu \mathrm{s},(b) t_{2}=1 \mu \mathrm{s},(c) t_{3}=6 \mu \mathrm{s}$ and $(d) t_{4}=10 \mu \mathrm{s}$ for conditions given in Table 4.1.

tities $\hat{n}_{2}\left(n_{1}\right)$ and $\hat{n}_{3}\left(n_{1}\right)$ are defined as the location in the $\left(n_{2}, n_{3}\right)$-plane at which the length of the nucleation flux vector, $\left|\mathbf{J}_{\mathbf{n}}\right|$, has its maximum. After a certain induction time, $\tau$, the solution attains its steady state and for this specific case $\tau \approx 10 \mu s$. Due to the high forward rate of ethanol $(k=1)$ the main flux bypasses the saddle point similarly as obtained in the theoretical and numerical studies of Refs. [54, 74] and Ref. [53], respectively.

We analyze the total nucleation flux normal to the $\left(n_{2}, n_{3}\right)$-plane as a function of $n_{1}$ and time $t$ defined as

$$
\bar{J}_{n_{1}}(t) \equiv \sum_{n_{2}, n_{3}} J_{\mathbf{n}}^{1}(t)
$$

Fig. 4.8 shows the time dependence of $\bar{J}_{n_{1}}$. We see a similar dependence of the total nucleation flux at different values of $n_{1}$ as found in single component nucleation [2]. After $\tau \approx 10 \mu s$ the nucleation fluxes converge to their steady state value, which is 


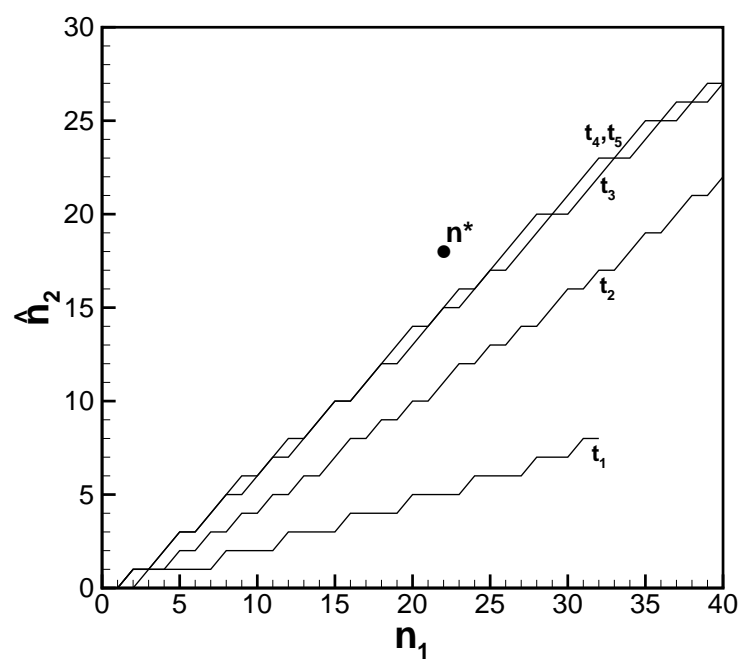

(a)

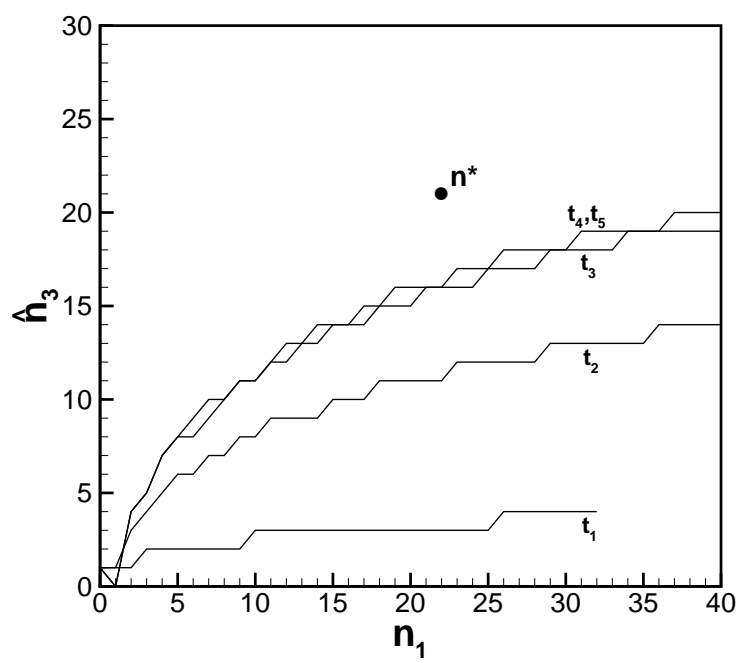

(b)

FIGURE 4.7: The main nucleation path (a) $\hat{n}_{2}$ and $(b) \hat{n}_{3}$ as a function of $n_{1}$ at $t_{1}=$ $0.1 \mu \mathrm{s}, t_{2}=1 \mu \mathrm{s}, t_{3}=4 \mu \mathrm{s}, t_{4}=6 \mu \mathrm{s}$ and $t_{5}=10 \mu \mathrm{s}$. The dot denotes the saddle point $\mathbf{n}^{*}$.

consistent with the previously presented induction time. 


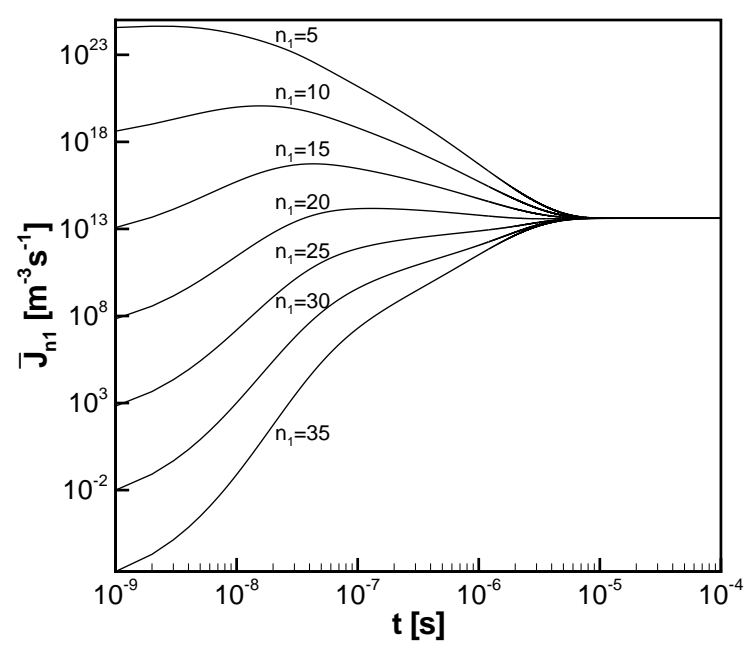

FiguRE 4.8: Time dependence of $\bar{J}_{n_{1}}$ at $n_{1}=5,10, \ldots, 35$.

\subsection{Conclusions}

We have presented a geometric multigrid algorithm for $N$-component nucleation. The algorithm clearly demonstrates the feasibility of multigrid as faster alternative solution method for numerically solving the NBD equations. The proposed method is a first step in the development of an optimally efficient multigrid solution method for $N$-component condensation. The success of the algorithm relies largely on the appropriate choice of the restriction and interpolation operators ensuring that the coarse grid operators "inherit" the fine grid error smoothing and stability properties of the fine grid operator. For the TBD equations an efficiency increase of a factor of $5-10$ is obtained, compared to simple single grid iteration.

The results show that in the initial stages of the nucleation process the main nucleation path is far from the saddle point of the free energy of formation. Moreover, the steady state solution shows a deviation of the main nucleation flux from the saddle point, contrary to the assumptions made in the classical nucleation theory. In spite of this, simulations show that the steady state nucleation rate itself is predicted within one order of magnitude by the analytic expression given in Refs. [78, 81]. Furthermore, time dependent nucleation rate data show an induction time of $10 \mu \mathrm{s}$ for the ternary mixture considered. These observations can aid in the development and validation of $N$-component nucleation theories.

The algorithm is not restricted to vapor-liquid transition and is expected to work for a wide variety of formation problems. The results presented here only considered the cluster size distribution for relatively small clusters. If the size distribution is 
desired in a large cluster domain, a non-uniform grid in the $\mathbf{n}$-space is mandatory which complicates the construction of a geometric multigrid algorithm. For these cases an Algebraic Multigrid (AMG) algorithm [75] could be advantageous. Note that in the present study a non-depletable vapor is assumed. Including the vapor depletion involves a global constraint, which affects the forward and backward rates in the operator $L[16]$. 



\title{
Stationary Diffusion FluX MODEL
}

\begin{abstract}
A new model describing the evolution of clusters in the processes of single component nucleation and growth is proposed. The diffusion flux in the non-stationary FokkerPlanck equation with an unknown distribution function is approximated by the closed form expression containing the steady-state solution of the Zeldovich-Frenkel equation. This is justified due to the smallness of the induction time of cluster formation compared to the timescale observed in experiments. The resulting Stationary Diffusion Flux model is valid for all cluster sizes, computationally efficient and applicable to various types of cluster formation processes. Its application to a nucleation pulse experiment shows excellent agreement with the solution of the set of formally exact Becker-Döring equations.
\end{abstract}

The work in this chapter has been published in revised form as: D.S. van Putten and V.I. Kalikmanov, J. Chem. Phys. 130, 164508 (2009).

\subsection{Introduction}

Modeling the cluster size distribution, describing the evolution of clusters in the processes of unary nucleation and growth, is an important subject in various areas of physics. Applications can be found in the fields of condensing vapors [20], crystallization [18, 61], ferromagnetics [57], aerosol and atmospheric science [17], combustion science [41] and chemical processes [26]. In these fields an accurate solution of the Becker-Döring (BD) equations [4], or its continuous equivalent, i.e. the FokkerPlanck equation (FPE) [85], is mandatory. Both BD and FPE couple the processes of nucleation and growth and as such provide the size distribution in the entire cluster size domain. However, this leads to excessive computation times.

The urge for computationally more tractable models has led to the introduction of the General Dynamic Equation (GDE) [52]. The GDE decouples the nucleation and growth process and models nucleation by means of a Dirac $\delta$-function term. In its 
original form the source term introduces clusters at the critical size [52]. At fixed external conditions, however, the newly born clusters of critical size, $n^{*}$ are in unstable equilibrium and therefore do not grow. This results in a cluster size distribution which is singular at $n^{*}$. Moreover, a rapid change in external conditions resulting in an increase of the critical size, leads to evaporation of all clusters. Recent studies made an attempt to overcome these deficiencies of the GDE by replacing the $\delta$-function by a boundary condition at a certain size larger than the critical one $[22,46,66]$. The GDE is aimed at the description of large supercritical clusters and does not supply the cluster size distribution in the subcritical region.

The aim of this chapter is to present a simplification of the FPE, which is valid for the entire cluster size range without the need for additional restraints. In general, the flux in the FPE contains a drift term and a diffusion term. The approximation refers to the diffusion flux, which is pronounced in the range of cluster sizes up to a certain size slightly exceeding the critical cluster size. It transports the newly formed clusters to the supercritical range and becomes negligibly small compared to the drift flux outside of this domain [60]. Due to the extreme small value of the induction time of nucleation [2], $t_{\text {ind }},\left(\sim 10^{-6} \mathrm{~s}\right)$ compared to laboratory time scales, the size distribution in the diffusion flux can be replaced by the stationary solution of the ZeldovichFrenkel equation $[32,85]$. This leads to an analytical expression for the diffusion flux. The result is termed the Stationary Diffusion Flux (SDF) model. The stationary cluster size distribution can still change with time due to its implicit dependence on the external conditions and therefore the SDF is capable of treating time-dependent condensation problems.

\subsection{Stationary Diffusion Flux model}

In the continuous limit the BD equations reduce to the FPE [85] for the continuous number density, $\rho(n, t)$ :

$$
\begin{aligned}
\frac{\partial \rho(n, t)}{\partial t} & =-\frac{\partial J(n)}{\partial n}, \quad \text { with } \\
J(n) & =-D(n) \frac{\partial \rho(n, t)}{\partial n}+\dot{n}(n) \rho(n, t) .
\end{aligned}
$$

The first term on the right-hand side of Eq. (5.2) is the diffusion flux in size space while the second one is the drift flux containing the growth rate $\dot{n}(n)$. The diffusion coefficient $D(n)$ and the growth rate $\dot{n}(n)$ are related by the fundamental Zeldovich relation [32]

$$
D(n)=-\frac{\dot{n}(n)}{g^{\prime}(n)}, \quad \text { with } \quad g^{\prime}(n)=\frac{d g(n)}{d n} .
$$

It is important to note that at the critical cluster size, $n^{*}, g(n)$ is at its maximum implying that $g^{\prime}\left(n^{*}\right)=0$. The growth rate at the critical size, i.e. $\dot{n}\left(n^{*}\right)$ also vanishes, 
however $D\left(n^{*}\right)$ remains finite. The general form of the law for the growth rate $\dot{n}$ can be written in terms of the reduced radius $r \equiv\left(n / n^{*}\right)^{\frac{1}{3}}$ as in Ref. [62],

$$
\dot{r}(r)=\frac{1}{\tau r^{\alpha}}\left(1-\frac{1}{r}\right), \quad \text { with }\left.\quad \tau^{-1} \equiv \frac{d \dot{n}}{d n}\right|_{n^{*}},
$$

where the power index $\alpha$ depends on the type of mass exchange, e.g. $\alpha=0$ and 1 correspond to the ballistic and diffusion-limited cluster growth, respectively.

The starting point of the SDF is the Fokker-Planck equation. We propose an approximation in which the diffusion term in Eqs. (5.1)-(5.2), involving the unknown cluster size distribution, $\rho(n, t)$, is approximated by

$$
Q_{\mathrm{s}} \equiv \frac{\partial}{\partial n}\left\{D(n) \frac{\partial \rho_{\mathrm{s}}(n)}{\partial n}\right\}
$$

containing the stationary flux of nucleating clusters; here $\rho_{\mathrm{s}}(n)$ is the non-equilibrium stationary cluster size distribution. The latter is defined as the steady-state solution of the Zeldovich-Frenkel equation [32]

$$
\frac{\partial}{\partial n}\left\{D(n) \rho^{\mathrm{eq}}(n) \frac{\partial \gamma_{\mathrm{s}}(n)}{\partial n}\right\}=0, \quad \gamma_{\mathrm{s}}(n) \equiv \frac{\rho_{\mathrm{s}}(n)}{\rho^{\mathrm{eq}}(n)},
$$

where $\rho^{\mathrm{eq}}(n)$ is the equilibrium size distribution (Eq. (3.7)). Integrating Eq. (5.6) using the boundary conditions $\gamma_{\mathrm{s}}(1)=1$ and $\lim _{n \rightarrow \infty} \gamma_{\mathrm{s}}(n)=0$, yields [32]

$$
\rho_{\mathrm{S}}(n)=\rho^{\mathrm{eq}}(n) J_{\mathrm{s}} \int_{n}^{\infty} \frac{d \tilde{n}}{D(\tilde{n}) \rho^{\mathrm{eq}}(\tilde{n})},
$$

where the steady-state nucleation rate $J_{\mathrm{s}}$ is

$$
J_{\mathrm{s}}=\left[\int_{1}^{\infty} \frac{d n}{D(n) \rho^{\mathrm{eq}}(n)}\right]^{-1} .
$$

Combining Eq. (5.5) with (5.2), the latter for the stationary conditions, $\rho(n, t)=\rho_{\mathrm{s}}(n)$ and $J_{\mathrm{s}}=$ const, we find

$$
Q_{\mathrm{s}}=\frac{\partial}{\partial n}\left\{\dot{n} \rho_{\mathrm{s}}\right\}=\frac{\partial}{\partial n}\left\{\dot{n} \rho^{\mathrm{eq}}\left[1-J_{\mathrm{s}} \varepsilon(n)\right]\right\},
$$

with

$$
\varepsilon(n) \equiv \int_{1}^{n} \frac{d \tilde{n}}{D(\tilde{n}) \rho^{\mathrm{eq}(\tilde{n})}} .
$$


This leads to the general expression for the SDF

$$
\frac{\partial \rho}{\partial t}+\frac{\partial}{\partial n}(\dot{n} \rho)=\frac{\partial}{\partial n}\left\{\dot{n} \rho^{\mathrm{eq}}\left[1-J_{\mathrm{s}} \varepsilon(n)\right]\right\},
$$

which is valid for all cluster sizes. In the limit of "small" $n$, i.e. $n \ll n^{*}$, such that $g(n) \ll g\left(n^{*}\right)$, the free energy $g(n)$ is dominated by the positive surface contribution implying that the number of small clusters continues to have its equilibrium value in spite of the drift flux.

Using Eq. (5.9), we can rewrite the SDF model as a homogeneous conservation equation for size distribution as

$$
\frac{\partial \rho}{\partial t}+\frac{\partial}{\partial n}\left(\dot{n}_{\mathrm{SDF}} \rho\right)=0
$$

where the effective SDF growth rate $\dot{n}_{\mathrm{SDF}}(\rho, n)$ incorporates the stationary diffusion flux and is defined as

$$
\dot{n}_{\mathrm{SDF}}(\rho, n) \equiv \dot{n}\left(1-\frac{\rho_{\mathrm{S}}}{\rho}\right) .
$$

The behavior of the size distribution can be analyzed by considering Eq. (5.13). If $\rho>\rho_{\mathrm{S}}, \dot{n}_{\mathrm{SDF}}$ behaves similar as the deterministic growth rate $\dot{n}$. This situation occurs when the supersaturation is decreased and consequently the stationary size distribution decreases. For $\rho \gg \rho_{\mathrm{S}}$ the distribution behaves as if the diffusion flux is absent. On the other hand when $\rho<\rho_{\mathrm{s}}$, e.g. when the supersaturation is increased, this behavior inverts. In the subcritical cluster size region the clusters grow until the steady state distribution is attained.

One can simplify Eq. (5.11) in the domain $n \geq n^{*}$ using the properties of $\rho^{\mathrm{eq}}(n)$. The function $\exp (g(n))$ has a strong maximum at $n=n^{*}$, implying that we can expand $g(n)$ around $n^{*}$ up to the second order term,

$$
\begin{aligned}
g(n) & \approx g\left(n^{*}\right)+\frac{1}{2} g^{\prime \prime}\left(n^{*}\right)\left(n-n^{*}\right)^{2}, \quad \text { with } \\
g^{\prime \prime}\left(n^{*}\right) & =\left.\frac{\partial^{2} g}{\partial n^{2}}\right|_{n^{*}}<0 .
\end{aligned}
$$

The diffusion coefficient, $D(n)$ is proportional to the cluster size as $n^{\frac{2-\alpha}{3}}$ (e.g. for the ballistic growth law $\left.1 / D(n) \propto n^{-\frac{2}{3}}\right)$. Therefore, in view of the exponential function in $\rho^{\mathrm{eq}}(n)$, the main contribution of the diffusion coefficient in the integral of Eq. (5.7) is for values of $n$ close to the lower integration limit, yielding

$$
\rho_{\mathrm{s}}(n) \approx J_{s} \frac{\exp (-g(n))}{D(n)} \int_{n}^{\infty} \exp (g(\tilde{n})) d \tilde{n} .
$$


Substituting Eq. (5.15) into (5.9) and using (5.3) results in

$$
Q_{\mathrm{s}}=J_{\mathrm{s}} \frac{\partial}{\partial n}\left\{-g^{\prime}(n) \exp (-g(n)) \int_{n}^{\infty} \exp (g(\tilde{n})) d \tilde{n}\right\} .
$$

Substituting these relations in Eq. (5.16) and defining the Zeldovich factor, $\mathcal{Z} \equiv$ $\sqrt{-g^{\prime \prime}\left(n^{*}\right) / 2 \pi}$, we find

$$
\begin{aligned}
Q_{\mathrm{s}} & =J_{\mathrm{s}} \frac{\partial}{\partial n}\left\{\sqrt{\pi} \zeta \exp \left(\zeta^{2}\right) \operatorname{erfc}(\zeta)\right\}, \quad \text { with } \\
\zeta & \equiv \sqrt{\pi} \mathcal{Z}\left(n-n^{*}\right),
\end{aligned}
$$

where $\operatorname{erfc}(x)$ is the complementary error function [1]. For large clusters

$$
\lim _{\zeta \rightarrow \infty}\left\{\sqrt{\pi} \zeta \exp \left(\zeta^{2}\right) \operatorname{erfc}(\zeta)\right\}=1 .
$$

yielding $Q_{\mathrm{s}} \rightarrow 0$ for $n \rightarrow \infty$ as required [60]. Evaluation of Eq. (5.17) yields

$$
\begin{aligned}
Q_{\mathrm{s}} & =J_{\mathrm{s}} \pi \mathcal{Z} \Psi(\zeta), \quad \text { with } \\
\Psi(\zeta) & =-\frac{2 \zeta}{\sqrt{\pi}}+\left(1+2 \zeta^{2}\right) \exp \left(\zeta^{2}\right) \operatorname{erfc}(\zeta) .
\end{aligned}
$$

At the critical cluster size $\Psi(\zeta=0)=1$ and decreases rapidly with $\zeta$, demonstrating the vanishing of the diffusion term at large $n$. Finally, in the supercritical cluster size domain the SDF, i.e. Eq. (5.11), reduces to

$$
\frac{\partial \rho}{\partial t}+\frac{\partial}{\partial n}(\dot{n} \rho)=J_{\mathrm{s}} \pi \mathcal{Z} \Psi(\zeta), \quad \zeta \geq 0
$$

where $\zeta$ is given by Eq. (5.18). Eq. (5.20) is a convenient approximate form for nucleation experiments, in which the supercritical clusters are of major interest. The right-hand side represents the source term with newly born clusters and shows resemblance with the GDE [52], which reads

$$
\frac{\partial \rho}{\partial t}+\frac{\partial}{\partial n}(\dot{n} \rho)=J_{\mathrm{s}} \delta\left(n-n^{*}\right) .
$$

The difference, however, is that the source term of Eq. (5.20) takes into account all supercritical clusters and not just the critical size $n^{*}$. This prevents the appearance of a singular size distribution in case the external conditions are fixed.

The general SDF Eq. (5.11) is applicable to all cluster sizes in the range $1 \leq n<\infty$ and is solved by imposing an initial condition and by specifying the thermodynamic model of cluster formation, $g(n)$, as well as the growth law, $\dot{n}(n)$. Moreover, additional constraints are not necessary for the subcritical clusters. 


\subsection{Results and discussion}

We apply the SDF to a nucleation pulse experiment (see e.g. Refs. $[33,79])$. The test case consists of a typical stepwise constant pressure-temperature profile comprising a region with high supersaturation, in which nucleation and growth take place (region $I$ ), followed by a region with low supersaturation, in which nucleation is negligible (region II). An example of such an experiment is presented in Table 5.1. The pulse duration is $50 \mu \mathrm{s}$. The supersaturation is the single component equivalent of the vapor activity and is given by $S=y p / p^{s}(T)$, where $p$ is the total pressure, $y$ is the non-equilibrium vapor fraction and $p^{s}(T)$ is the saturation pressure of the nucleating substance. We take water as nucleating substance with $y=7 \cdot 10^{-3}$; its thermodynamic properties are those of Ref. [79]. The induction time of nucleation is defined as $t_{\text {ind }}=\left(4 \pi D\left(n^{*}\right) \mathcal{Z}^{2}\right)^{-1}$, (see e.g. Ref. [2]).

\begin{tabular}{lcc}
\hline Parameter & $\begin{array}{c}\text { Region I } \\
(t \leq 50 \mu \mathrm{s})\end{array}$ & $\begin{array}{c}\text { Region II } \\
(50 \mu \mathrm{s}<t<300 \mu \mathrm{s})\end{array}$ \\
\hline$p[\mathrm{kPa}]$ & 77.0 & 115.5 \\
$T[\mathrm{~K}]$ & 240.0 & 270.0 \\
$S$ & 14.34 & 1.67 \\
$n^{*}$ & 23 & 1885 \\
$\log _{10} J_{\mathrm{s}}\left[\mathrm{m}^{-3} \mathrm{~s}^{-1}\right]$ & 16.6 & -180 \\
$t_{\text {ind }}[\mu \mathrm{s}]$ & 1.7 & 29.2 \\
\hline
\end{tabular}

TABLE 5.1: Nucleation pulse test case conditions for water with vapor fraction $y=$ $7 \cdot 10^{-3}$.

The results are validated utilizing the solution of the set of formally exact BD equations (3.1), with the forward and backward rates determined as described in Chapter 3. The monomer density is non-depleting and assumed constant in both regions. A set of $10^{5}$ BD equations is solved using the Piecewise Constant Flux approximation [11]. The SDF is solved employing the van Leer MUSCL scheme (see [30] and references therein). Using the SDF model, the computational effort is reduced by a factor 8 compared to the effort required for solution of the BD equations. For consistent comparison of the present solution with that of the $\mathrm{BD}$ equations, the growth law in the SDF should be chosen appropriately $[58,59]$ as

$$
\dot{n}(n)=D(n)\left[1-\exp \left(g^{\prime}(n)\right)\right] .
$$

Fig. 5.1 depicts the cluster size distribution in region $I$ at several time instants. The results from the SDF show a good agreement with those of the BD equations for all cluster sizes. A small difference between the solutions at $t_{1}$ is due to the instantaneous occurrence of the pulse at $t=0$. The BD solution exhibits a certain 


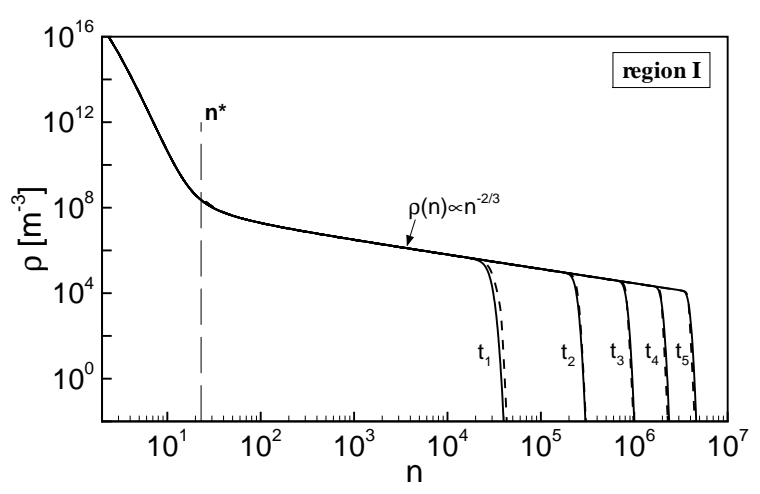

Figure 5.1: Cluster size distribution $\rho(n, t): B D$ equations (solid) and SDF (shortdashed) for nucleation pulse experiment in region I at $t_{1}=10 \mu \mathrm{s}, t_{2}=20 \mu \mathrm{s}, t_{3}=$ $30 \mu \mathrm{s}, t_{4}=40 \mu \mathrm{s}$ and $t_{5}=50 \mu \mathrm{s}$. The vertical long-dashed line shows the location of the critical cluster.

relaxation time to be noticed in the supercritical domain. For times larger than $t_{1}$ the difference becomes negligible. At $n \gg n^{*}$ the diffusion term vanishes and at large $t$, $\rho(n, t) \approx \rho_{\mathrm{s}}(n) \approx J / \dot{n}(n)$ until the double exponential decay of the front of the size distribution occurs, as described by Shneidman [62].

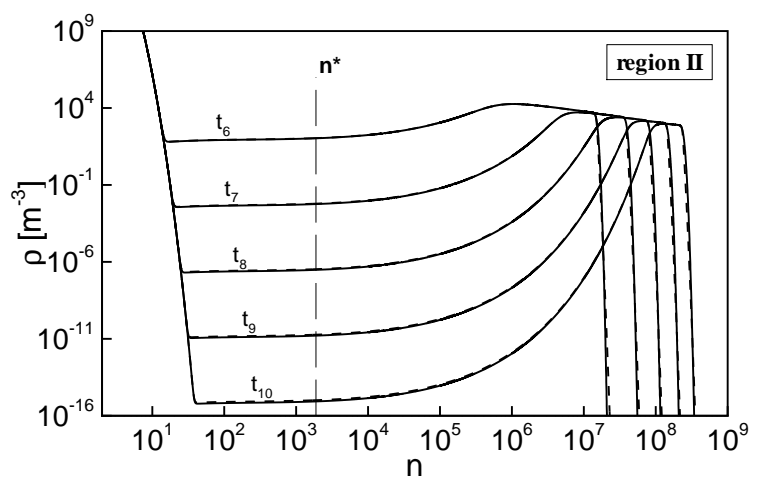

Figure 5.2: Cluster size distribution $\rho(n, t)$ in region II at $t_{6}=100 \mu \mathrm{s}, t_{7}=150 \mu \mathrm{s}$, $t_{8}=200 \mu \mathrm{s}, t_{9}=250 \mu \mathrm{s}$ and $t_{10}=300 \mu \mathrm{s}$. Other notations - see Fig. 5.1.

The results for the SDF in region II (Fig. 5.2) are in excellent agreement with the BD solutions in the entire cluster size domain. The size distribution in the region of $n \ll n^{*}$ resembles the equilibrium size distribution, as observed in Eq. (5.11). In region II, the SDF reduces to the conservation equation for $\rho(n, t)$ in the supercritical region. We find that at the critical clusters $\rho\left(n^{*}, t\right)$ decays exponentially with time - in 
agreement with Ref. [63]. Furthermore, the SDF solution exhibits a general feature: the exponential decay of $\rho(n, t)$ with time is true for the subcritical clusters up to the equilibrium size distribution. This test case demonstrates the validity of the SDF for the entire cluster range.

The solution of the SDF can be used for validation of various types of nucleation experiments (e.g. nucleation pulse tubes, diffusion cloud chambers and expanding nozzle flows [33, 72]). For continuously varying pressure-temperature profiles [8] all $p, T$-dependent functions - $J, S, g(n)$ and $\rho_{\mathrm{s}}(n)$ - become parametric functions of time.

From the size distribution, the evolution with time of the total number of formed clusters can be calculated. For the nucleation pulse experiment of Table 5.1, a portion of the clusters formed in region I evaporates ( 10\%). This is due to the rapid increase of the critical size in region II, causing a part of the size distribution to become subcritical. This correction can be applied to experimentally measured nucleation rates, as done by Ref. [22]. Moreover, the SDF can serve as a guideline for designing nucleation pulse experiments. Important parameters in these experiments are the pulse duration time (region I) and the ratio between the supersaturation in both regions. Additionally, an accurate description of the shape of the cluster size distribution provides useful information for the applied measurement techniques.

\subsection{Conclusions}

The evolution of clusters in the processes of nucleation and growth can be approximated quite well by the SDF. The model is concise and capable of treating any time dependent nucleation problem under the assumption that the timescales of the experiment are much longer than the induction time of nucleation. The SDF does not invoke additional constraints on the cluster size distribution and can be applied to all cluster sizes. For most practical applications, in which the supercritical clusters are of major importance, the simplified SDF for $n \geq n^{*}$ can be used (Eq. (5.20)). Note that the elaboration in this chapter has been carried out for a purely phenomenological model of the free energy of cluster formation. The validation of the SDF has been demonstrated for a nucleation pulse experiment. It is expected that SDF may be of general validity for any cluster formation process. 


\section{Phase Path Analysis ALGORITHM FOR THE NGDE}

A computationally efficient method is proposed for the calculation of the $\mathrm{N}$-component cluster size distribution. This method is based on the Phase Path Analysis algorithm, which originally has been derived for single component condensation. First this method is extended by considering the $N$-component General Dynamic Equation (NGDE). The NGDE introduces clusters at a point in $\mathbf{n}$-space. The location of this source point is determined by the Gibbs free energy of formation and the impingement rates of the constituents. The resulting model describes the $N$-component cluster size distribution along a line in $\mathbf{n}$-space. For a typical nucleation pulse experiment the solution of the Binary General Dynamic Equation is compared with the solution of formally exact Binary Becker-Döring equations. The results show good agreement for the cluster composition, size and the integral properties of the size distribution.

The work in this chapter has been published in revised form as: D.S. van Putten, R.S.R. Sidin and R. Hagmeijer, J. Chem. Phys. 132, 184511 (2010).

\subsection{Introduction}

The calculation of the cluster size distribution in $N$-component nucleating systems is of great interest in many fields of physics. To obtain the $\mathrm{N}$-component cluster size distribution the full set of NBD equations need to be solved. The solution of the binary condensation problem has been addressed in several studies $[35,65,81]$ and more recently the ternary problem has been solved in a confined cluster region [47] and Chapter 4. This solution method requires excessive computational time and is not attractive for most practical applications in which the cluster sizes range from 10 to $10^{7}$ molecules.

For single component systems the General Dynamic Equation (GDE) has been introduced [52], which is a computationally efficient model for the calculation of the unary size distribution. The GDE decouples the processes of nucleation and growth 
and as a result this model is not applicable to the size distribution in the region of subcritical cluster sizes. On the other hand, recent studies have demonstrated that for large supercritical clusters the global behavior of the distribution is in good agreement with the solution of the formally exact Becker-Döring equations [63, 66]. Some ambiguity still exists, however, in the choice of the location of the source term in the $\mathbf{n}$-space. This will be addressed accordingly by using the Stationary Diffusion Flux (SDF) model [45] described in Chapter 5.

First the GDE is extended to $N$-component systems by using quasi-1D considerations of the SDF model. The resulting $N$-component General Dynamic Equation (NGDE) is a conservation equation for the $N$-component size distribution with a source term located at a point in $\mathbf{n}$-space. The location of the source term is derived from the Gibbs free energy of formation and the impingement rates of the constituents. The binary case is addressed to allow for a geometric construction and serves as an illustrative case of the NGDE due to the reduction in dimensionality.

Subsequently, an efficient solution algorithm is introduced which exploits the hyperbolic character of the NGDE for the $N$-component size distribution. The algorithm is an extension of the Phase Path Analysis (PPA) algorithm derived for unary systems [20]. It is shown that the resulting cluster size distribution, which has a Dirac $\delta$-function character, agrees well in integral sense with the solution of the NBD equations.

\section{2 $N$-component General Dynamic Equation}

The continuous equivalent of the $N$-component Becker-Döring (NBD) equations (3.1) is the $N$-component Fokker-Planck Equation (NFPE) which describes the time evolution of the continuous $N$-component cluster distribution $\rho(\mathbf{n}, t)$ [85]

$$
\frac{\partial \rho(\mathbf{n}, t)}{\partial t}=-\nabla_{\mathbf{n}} \cdot \mathbf{J}(\mathbf{n}),
$$

where $\nabla_{\mathbf{n}}=\left(\frac{\partial}{\partial n_{1}}, \frac{\partial}{\partial n_{2}}, \ldots, \frac{\partial}{\partial n_{N}}\right)^{\mathrm{T}}$ and the nucleation flux vector $\mathbf{J}(\mathbf{n})=\left(J_{1}(\mathbf{n}), J_{2}(\mathbf{n})\right.$, $\left.\ldots, J_{N}(\mathbf{n})\right)^{\mathrm{T}}$ is given by

$$
\begin{aligned}
\mathbf{J}(\mathbf{n}) & =-\rho^{\mathrm{eq}}(\mathbf{n}) \mathrm{D}(\mathbf{n}) \nabla_{\mathbf{n}} \gamma(\mathbf{n}) \\
& =-\mathrm{D}(\mathbf{n}) \nabla_{\mathbf{n}} \rho(\mathbf{n})+\dot{\mathbf{n}}(\mathbf{n}) \rho(\mathbf{n})
\end{aligned}
$$

where $\gamma(\mathbf{n}) \equiv \rho(\mathbf{n}) / \rho^{\mathrm{eq}}(\mathbf{n})$. The first term on the right-hand side of Eq. (6.3) is the diffusion flux vector with the diffusion tensor $\mathrm{D}(\mathbf{n})$, the second term is the drift flux vector containing the growth rate vector $\dot{\mathbf{n}}(\mathbf{n})$, with $\dot{n}_{k}(\mathbf{n}, t)$ given by the growth law in $k$-direction. The latter is obtained from the $N$-component generalization of the fundamental Zeldovich relation [85]

$$
\dot{\mathbf{n}}(\mathbf{n})=\mathrm{D}(\mathbf{n}) \nabla_{\mathbf{n}}\left[\ln \rho^{\mathrm{eq}}(\mathbf{n})\right] .
$$


If the normalization constant $\rho^{0}(\mathbf{n})$ in the continuous form of Eq. (3.7) is not a function of cluster size, Eq. (6.4) reduces to $\dot{\mathbf{n}}(\mathbf{n})=-\mathrm{D}(\mathbf{n}) \nabla_{\mathbf{n}} g(\mathbf{n})$ with $g(\mathbf{n})$ the Gibbs free energy form Eq. (3.8).

We define the supercritical cluster size region $\Omega^{*}$ in $\mathbf{n}$-space as given by Eq. (3.17). The $N$-component General Dynamic Equation (NGDE) effectively decouples the processes of nucleation and growth and introduces the newly formed clusters at a size, $\mathbf{n}_{0} \in \Omega^{*}$, at a rate equal to the steady state nucleation rate, $J_{\mathrm{s}}$. The NGDE is constructed by neglecting the diffusion term in the NFPE and by introducing the newly formed clusters in the supercritical cluster size region by means of a Dirac $\delta$-function [46]

$$
\frac{\partial \rho}{\partial t}+\nabla_{\mathbf{n}} \cdot(\dot{\mathbf{n}} \rho)=J_{\mathrm{s}} \delta\left(\mathbf{n}-\mathbf{n}_{0}\right)
$$

The $N$-component classical nucleation theory [74] provides $J_{\mathrm{s}}$ and the aim of this chapter is to define the location of the 'source point' $\mathbf{n}_{0}$. The location of $\mathbf{n}_{0}$ is deduced from the SDF model and is based on the closed form expression for the steady state cluster size distribution.

The diffusion flux in Eq. (6.3) reduces rapidly as the cluster size passes the saddle point $[45,46]$. The source location $\mathbf{n}_{0}$ is therefore expected to be located near the critical size $\mathbf{n}^{*}$. To aid the computation of the steady state distribution a new coordinate system is defined by $\boldsymbol{\xi}=\mathrm{A}^{-1} \Delta \mathbf{n}$ with $\Delta \mathbf{n}=\mathbf{n}-\mathbf{n}^{*}$. The transformation is aimed at removing the anisotropy of the nucleation flux (i.e. the diffusion tensor $\mathrm{D}(\mathbf{n})$ ) in Eq. (6.2). Moreover, it is used to facilitate the integration of the equilibrium size distribution. The divergence of the steady state nucleation flux transforms as

$$
\nabla_{\mathbf{n}} \cdot\left(\rho^{\mathrm{eq}} \mathrm{D} \nabla_{\mathbf{n}} \gamma_{\mathrm{s}}\right)=\nabla_{\xi} \cdot\left(\rho^{\mathrm{eq}} \mathrm{A}^{-1} \mathrm{DA}^{-\mathrm{T}} \nabla_{\xi} \gamma_{\mathrm{s}}\right)=0
$$

where $\gamma_{\mathrm{s}}(\boldsymbol{\xi})=\rho_{\mathrm{s}}(\boldsymbol{\xi}) / \rho^{\mathrm{eq}}(\boldsymbol{\xi})$ with $\rho_{\mathrm{s}}(\boldsymbol{\xi})$ the steady state size distribution. Near $\mathbf{n}^{*}$ we can expand $g(\mathbf{n})$ around $\mathbf{n}^{*}$ up to the second order, yielding the quadratic form

$$
g(\mathbf{n}) \approx g^{*}+\Delta \mathbf{n}^{\mathrm{T}} \mathrm{H}^{*} \Delta \mathbf{n},
$$

where $g^{*} \equiv g\left(\mathbf{n}^{*}\right)$ and $\mathrm{H}^{*}$ denotes the Hessian matrix of $\frac{1}{2} g(\mathbf{n})$ evaluated at $\mathbf{n}^{*}$

$$
\mathrm{H}_{i j}^{*}=\left.\frac{1}{2} \frac{\partial^{2} g(\mathbf{n})}{\partial n_{i} \partial n_{j}}\right|_{\mathbf{n}^{*}} .
$$

Application of the transformation to Eq. (6.7) yields

$$
g \approx g^{*}+\boldsymbol{\xi}^{\mathrm{T}} \mathrm{A}^{\mathrm{T}} \mathrm{H}^{*} \mathrm{~A} \boldsymbol{\xi} .
$$

Both Eq. (6.6) and Eq. (6.9) can be simplified by a process called simultaneous diagonalization [29]. Firstly, we assume that the diffusion tensor $\mathrm{D}(\mathbf{n})$ is approximately constant in the saddle point region, i.e. $\mathrm{D}(\mathbf{n}) \approx \mathrm{D}\left(\mathbf{n}^{*}\right) \equiv \mathrm{D}^{*}$. We use the following 
properties: $\mathrm{H}^{*}$ is symmetric and $\mathrm{D}^{*}$ is symmetric and positive-definite and define a symmetric matrix B as

$$
\mathrm{B} \equiv\left(\mathrm{D}^{*}\right)^{\frac{1}{2}} \mathrm{H}^{*}\left(\mathrm{D}^{*}\right)^{\frac{1}{2}},
$$

which can be written as

$$
\mathrm{B} \equiv \mathrm{U} \Lambda \mathrm{U}^{\mathrm{T}},
$$

where the columns of $\mathrm{U}$ form the orthonormal left eigenvectors of B. The entries $\lambda_{k}$ of the diagonal matrix $\Lambda$ are the eigenvalues of $\mathrm{B}$. Then, choosing the transformation matrix as

$$
\mathrm{A}=\left(\mathrm{D}^{*}\right)^{\frac{1}{2}} \mathrm{U}
$$

we obtain

$$
\begin{gathered}
A^{-1} D^{*} A^{-T}=U^{T}\left(D^{*}\right)^{-\frac{1}{2}} D^{*}\left(D^{*}\right)^{-\frac{1}{2}} U=I, \\
A^{T} H^{*} A=U^{T}\left(D^{*}\right)^{\frac{1}{2}} H^{*}\left(D^{*}\right)^{\frac{1}{2}} U=U^{T} B U=\Lambda .
\end{gathered}
$$

Hence, Eq. (6.6) becomes isotropic

$$
\nabla_{\xi} \cdot\left(\rho^{\mathrm{eq}} \nabla_{\xi} \gamma_{\mathrm{s}}\right)=0
$$

and the quadratic form of Eq. (6.9) reduces to a sum of squares

$$
g=g^{*}+\xi^{\mathrm{T}} \Lambda \xi=g^{*}+\sum_{k=1}^{N} \lambda_{k} \xi_{k}^{2} .
$$

Since B is symmetric, the eigenvalue problem Eq. (6.11) can be solved using the Jacobi method of successive orthogonal transformations, see e.g. Ref. [43]. The transformation matrix A is then determined by Eq. (6.12) and we denote the $k^{\text {th }}$ column of A by $\mathbf{q}_{k}$.

Using the terminology of Shneidman [54], the new coordinate system $\boldsymbol{\xi}$ consists of one 'unstable' coordinate $\xi_{1}$ in the direction of $\mathbf{q}_{1}$ corresponding to $\lambda_{1}<0$, i.e. the nucleation flux is in the direction of $\mathbf{q}_{1}$. All other 'stable' coordinates $\bar{\xi}=\left(\xi_{2}, \ldots, \xi_{N}\right)^{\mathrm{T}}$ correspond to $\lambda_{k}>0$ for $k=2, \ldots, N$. The combined coordinate system can be written as $\boldsymbol{\xi}=\left(\xi_{1}, \overline{\boldsymbol{\xi}}\right)^{\mathrm{T}}$.

The steady state nucleation rate and size distribution are calculated by considering Eq. (6.15) which reduces to the one-dimensional form

$$
\frac{\partial J_{\xi_{1}}}{\partial \xi_{1}}=0
$$

since the derivatives of $\mathbf{J}$ in all stable directions $\bar{\xi}$ vanish (direction of principle growth approximation). This means that in the saddle point region $J_{\xi_{1}}=J_{\xi_{1}}(\bar{\xi})$ which reads

$$
J_{\xi_{1}}(\overline{\boldsymbol{\xi}})=-\rho^{\mathrm{eq}}(\boldsymbol{\xi}) \frac{\partial \gamma_{\mathrm{s}}(\boldsymbol{\xi})}{\partial \xi_{1}},
$$


with the equilibrium size distribution in the transformed system given by

$$
\rho^{\mathrm{eq}}(\boldsymbol{\xi})=\rho_{*}^{\mathrm{eq}} \prod_{k=1}^{N} \exp \left(-\lambda_{k} \xi_{k}^{2}\right),
$$

with $\rho_{*}^{\mathrm{eq}}=\rho^{\mathrm{eq}}\left(\boldsymbol{\xi}^{*}\right)$. Integration of Eq. (6.18) over the unstable coordinate $\xi_{1}$ from $-\infty$ to $\xi_{1}$, yields

$$
1-\gamma_{\mathrm{s}}\left(\xi_{1}, \overline{\boldsymbol{\xi}}\right)=\frac{J_{\xi_{1}}(\overline{\boldsymbol{\xi}})}{\rho_{*}^{\mathrm{eq}}} \prod_{k=2}^{N} \exp \left(\lambda_{k} \xi_{k}^{2}\right) \int_{-\infty}^{\xi_{1}} \exp \left(\lambda_{1} \xi_{1}^{\prime 2}\right) d \xi_{1}^{\prime} .
$$

Evaluation of the integral leads to

$$
J_{\xi_{1}}(\overline{\boldsymbol{\xi}})=2 \sqrt{\frac{-\lambda_{1}}{\pi}} \rho_{*}^{\mathrm{eq}} \prod_{k=2}^{N} \exp \left(-\lambda_{k} \xi_{k}^{2}\right) \frac{1-\gamma_{\mathrm{s}}\left(\xi_{1}, \overline{\boldsymbol{\xi}}\right)}{\operatorname{erfc}\left(-\sqrt{-\lambda_{1}} \xi_{1}\right)},
$$

where $\operatorname{erfc}(x)$ is the complementary error function [1]. We observe that the left hand side is independent of $\xi_{1}$, and therefore defining

$$
\psi(\overline{\boldsymbol{\xi}}) \equiv \frac{1-\gamma_{\mathrm{s}}\left(\xi_{1}, \overline{\boldsymbol{\xi}}\right)}{\operatorname{erfc}\left(-\sqrt{-\lambda_{1}} \xi_{1}\right)}, \quad \text { with } \quad \frac{\partial \psi}{\partial \xi_{1}}=0
$$

Using the boundary condition $\gamma_{\mathrm{s}}\left(\xi_{1}, \overline{\boldsymbol{\xi}}\right)=0$ for $\xi_{1} \rightarrow \infty$ results in $\psi(\overline{\boldsymbol{\xi}})=\frac{1}{2}$ which immediately leads to

$$
\gamma_{\mathrm{s}}\left(\xi_{1}, \overline{\boldsymbol{\xi}}\right)=\frac{1}{2} \operatorname{erfc}\left(\sqrt{-\lambda_{1}} \xi_{1}\right)
$$

and since $\rho_{\mathrm{s}}(\boldsymbol{\xi})=\gamma_{s}(\boldsymbol{\xi}) \rho^{\mathrm{eq}}(\boldsymbol{\xi})$, yields

$$
\rho_{\mathrm{s}}(\boldsymbol{\xi})=\frac{1}{2} \rho_{*}^{\mathrm{eq}} \prod_{k=1}^{N} \exp \left(-\lambda_{k} \xi_{k}^{2}\right) \operatorname{erfc}\left(\sqrt{-\lambda_{1}} \xi_{1}\right) .
$$

Furthermore, Eq. (6.21) becomes

$$
J_{\xi_{1}}(\bar{\xi})=\sqrt{\frac{-\lambda_{1}}{\pi}} \rho_{*}^{\mathrm{eq}} \prod_{k=2}^{N} \exp \left(-\lambda_{k} \xi_{k}^{2}\right) .
$$

For the binary case the typical Gaussian shape of the nucleation flux in the $\left(n_{1}, n_{2}\right)$ space is illustrated in Fig. 6.1. The shape of the Gibbs free energy of formation is also indicated by iso-lines in the same figure.

The steady state nucleation rate is obtained by integrating Eq. (6.25) over all stable coordinates

$$
J_{\mathrm{s}}=\int_{\mathbb{R}^{N-1}} J_{\xi_{1}}(\bar{\xi}) \operatorname{det}(\mathrm{A}) d \xi_{2} d \xi_{3} \ldots d \xi_{N}=\pi^{(N-2) / 2} \frac{-\lambda_{1}}{\sqrt{\left|\operatorname{det}\left(\mathrm{H}^{*}\right)\right|}} \rho_{*}^{\mathrm{eq}},
$$




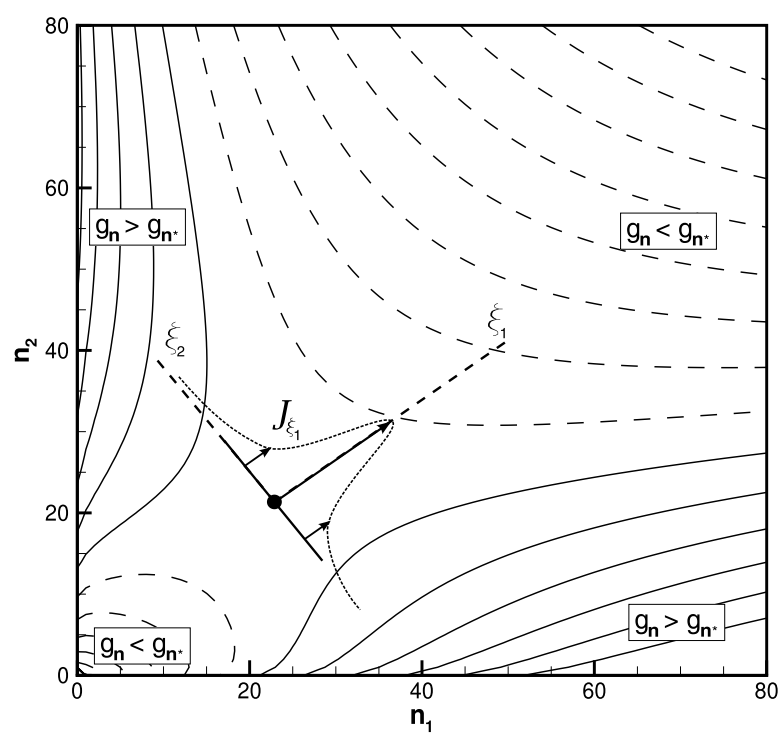

FigURE 6.1: Isocontours of Gibbs free energy of formation $g(\mathbf{n})$ in $\left(n_{1}, n_{2}\right)$-space. Solid iso-lines correspond to $g(\mathbf{n})>g\left(\mathbf{n}^{*}\right)$, dashed iso-lines correspond to $g(\mathbf{n})<$ $g\left(\mathbf{n}^{*}\right)$. The flux around $\mathbf{n}^{*}$ is given by $J_{\xi_{1}}\left(\xi_{2}\right)$ (dotted line) in the $\left(\xi_{1}, \xi_{2}\right)$-coordinate system. The dot indicates the saddle point $\mathbf{n}^{*}$.

where we used

$$
\operatorname{det}(\mathrm{U})=1, \quad \operatorname{det}(\Lambda)=\operatorname{det}\left(\mathrm{D}^{*}\right) \operatorname{det}\left(\mathrm{H}^{*}\right), \quad \operatorname{det}(\mathrm{A})=\sqrt{\operatorname{det}\left(\mathrm{D}^{*}\right)} .
$$

A more rigorous explanation of the integral in Eq. (6.26) is given in Appendix C.

We use the $N$-component generalization of the Stationary Diffusion Flux (SDF) approach for the quasi one-dimensional flux in the direction of the $\xi_{1}$-coordinate. The SDF approach involves replacing the diffusion flux in the FPE by its steady state value which is based on the small time scales in the nucleation process [45]. The nucleation flux in Eq. (6.25) has a strong exponential character in the direction of the stable coordinates $\bar{\xi}$ on the $\xi_{1}$-axis. Therefore, the source point $\mathbf{n}_{0}$ in Eq. (6.5) should be located on this axis. To account for the flux in the entire saddle point region we integrate Eq. (6.24):

$$
\overline{\rho_{\mathrm{s}}}\left(\xi_{1}\right) \equiv \int_{\mathbb{R}^{N-1}} \rho_{\mathrm{s}}\left(\xi_{1}, \overline{\boldsymbol{\xi}}\right) d \xi_{2} d \xi_{3} \ldots d \xi_{N} .
$$

Evaluation of Eq. (6.28) using Eq. (6.24) yields

$$
\begin{aligned}
\bar{\rho}_{\mathrm{s}}\left(\xi_{1}\right) & =\frac{1}{2} \rho_{*}^{\text {eq }} \frac{\pi^{(N-1) / 2} \sqrt{-\lambda_{1}}}{\sqrt{\left|\operatorname{det}\left(\mathrm{H}^{*}\right)\right|}} \exp \left(\zeta^{2}\right) \operatorname{erfc}(\zeta), \\
\zeta & \equiv \sqrt{-\lambda_{1}} \xi_{1} .
\end{aligned}
$$


Similarly to expressions (6.2) and (6.3), the nucleation flux in $\xi_{1}$-direction, given by Eq. (6.18), can be decomposed in a diffusion flux and a drift flux

$$
J_{\xi_{1}}(\bar{\xi})=-\frac{\partial \rho(\boldsymbol{\xi})}{\partial \xi_{1}}+\rho(\boldsymbol{\xi}) \frac{\partial}{\partial \xi_{1}}\left[\ln \rho^{\mathrm{eq}}(\boldsymbol{\xi})\right]
$$

We construct the quasi one-dimensional SDF by using Eq. (6.17) for steady state

$$
\frac{\partial J_{\xi_{1}}}{\partial \xi_{1}}=0, \quad \Rightarrow \quad \frac{\partial}{\partial \xi_{1}}\left\{-\frac{\partial \bar{\rho}_{\mathrm{s}}\left(\xi_{1}\right)}{\partial \xi_{1}}+\dot{\xi}_{1} \bar{\rho}_{\mathrm{s}}\left(\xi_{1}\right)\right\}=0,
$$

where $\dot{\xi}_{1} \equiv \frac{\partial}{\partial \xi_{1}}\left[\ln \rho^{\mathrm{eq}}(\boldsymbol{\xi})\right]$ is the growth rate in $\xi_{1}$-direction. Using Eq. (6.19) we obtain

$$
\dot{\xi_{1}}=\frac{\xi_{1}}{\tau_{\xi_{1}}}, \quad \text { with } \quad \tau_{\xi_{1}} \equiv-\frac{1}{2 \lambda_{1}},
$$

where $\tau_{\xi_{1}}$ is the Zeldovich time parameter, see also Appendix C. We approximate the diffusion flux in the quasi one-dimensional FPE (6.1) by the stationary diffusion flux as

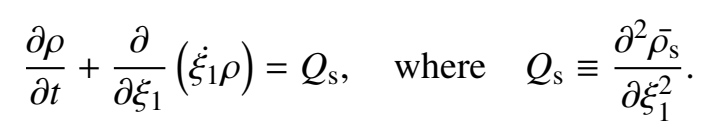

Using the steady state size distribution $\rho_{\mathrm{s}}\left(\xi_{1}\right)$ from Eq. (6.29), we obtain

$$
\begin{aligned}
Q_{\mathrm{s}} & =\pi^{(N-1) / 2} \rho_{*}^{\mathrm{eq}} \frac{\left(-\lambda_{1}\right)^{3 / 2}}{\sqrt{\left|\operatorname{det}\left(\mathrm{H}^{*}\right)\right|}} \Psi(\zeta), \\
\Psi(\zeta) & \equiv-\frac{2 \zeta}{\sqrt{\pi}}+\left(1+2 \zeta^{2}\right) \exp \left(\zeta^{2}\right) \operatorname{erfc}(\zeta),
\end{aligned}
$$

where the function $\Psi(\zeta)$ is identical to the expression derived by Refs. [45, 46] for $N=1,2$. Using the steady state nucleation rate, $J_{\mathrm{s}}$, from Eq. (6.26) yields

$$
Q_{\mathrm{s}}=J_{\mathrm{s}} \sqrt{-\lambda_{1} \pi} \Psi(\zeta)
$$

The function $\Psi(\zeta)$ is a monotonically decreasing function of $\zeta$ demonstrating the decrease of $Q_{\mathrm{s}}$ for $\zeta$ increasing in positive $\zeta$ direction, see Appendix C. At the critical size, $\Psi(\zeta=0)=1$. Define $\zeta_{0}$ such that it satisfies $\Psi\left(\zeta_{0}\right)=\epsilon \Psi(0)$, then $\epsilon$ is a measure for the decrease of the diffusion term. The choice of $\epsilon$ is a compromise; it should be sufficiently small for the diffusion term to be negligible compared to the drift term; on the other hand $\mathbf{n}_{0}$ should be located in the vicinity of $\mathbf{n}^{*}$, where the direction of principle growth approximation and the expansion in Eq. (6.7) are still valid. A value of $\epsilon=10^{-2}$ leads to $\zeta_{0}=3.6$, which value we will assume for calculations. We note that a similar analysis can be performed based on the ratio between the diffusion and drift fluxes, see Appendix C. The sensitivity of the NGDE to the choice of $\epsilon$ will be discussed in Section 6.4.1. If $\epsilon$ is chosen appropriately, the corresponding $\zeta_{0}$ is fixed 
and does not depend on the specific problem. In the original $\mathbf{n}$ coordinate system we note that $\Delta \mathbf{n}=\mathbf{q}_{1} \xi_{1}$ and using Eq. (6.29) yields the source location

$$
\mathbf{n}_{0}=\mathbf{n}^{*}+\frac{\zeta_{0}}{\sqrt{-\lambda_{1}}} \mathbf{q}_{1}
$$

Thus the location of the source point of the NGDE depends on the critical cluster size $\mathbf{n}^{*}$, the tolerance $\epsilon$ and the direction of $\mathbf{q}_{1}$ corresponding to the negative eigenvalue $\lambda_{1}$.

In the NGDE, the Gaussian profile in $\bar{\xi}$ is effectively replaced by a Dirac $\delta$-function at $\overline{\boldsymbol{\xi}}^{*}=0$ (i.e. $J_{\xi_{1}}(\overline{\boldsymbol{\xi}}) \approx J_{\mathrm{S}} \delta(\overline{\boldsymbol{\xi}})$ ). The construction with the Dirac $\delta$-function produces a flux, $J_{\tilde{\xi}}$ along the curvilinear coordinate system $\tilde{\xi}$ in $\mathbf{n}$-space. This is schematically illustrated in Fig. 6.2 for the binary GDE, i.e. $N=2$. Near the saddle point the direction of $J_{\tilde{\xi}}$ is approximately in $\xi_{1}$-direction. Similar effective 1D approaches have been proposed in Refs. [13, 54, 68].

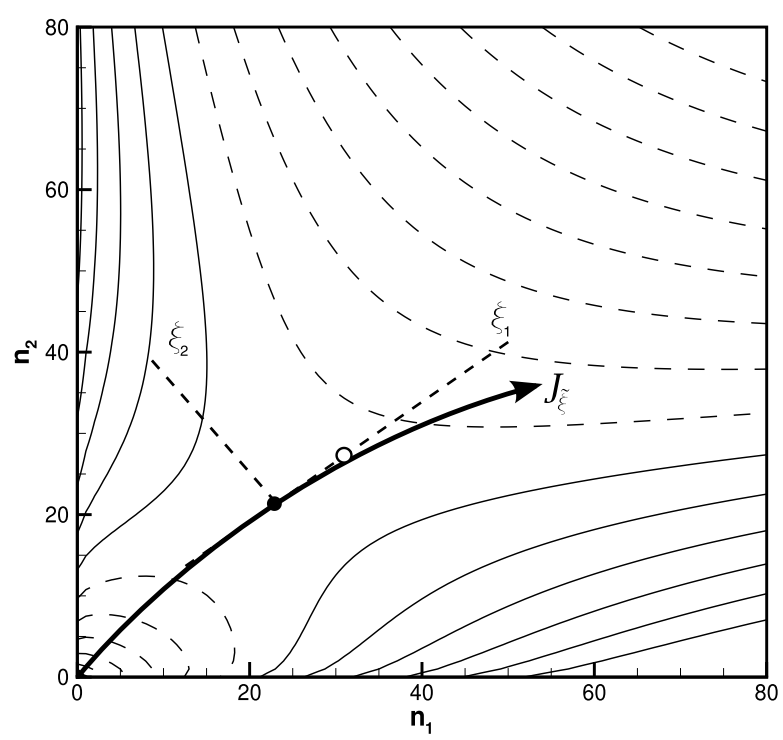

FIGURE 6.2: The open circle indicates the source point $\mathbf{n}_{0}$ and the solid arrow represents the flux $J_{\tilde{\xi}}$ in the curvilinear coordinate system $\tilde{\xi}$ in $\left(n_{1}, n_{2}\right)$-space. Other notations, see Fig. 6.1. 


\subsubsection{Special case of $N=1$}

For single component nucleation the procedure is simplified since the flux direction is evident. The SDF model [45] can be applied directly leading to

$$
n_{0}=n^{*}+\frac{\zeta_{0}}{\sqrt{\pi} \mathcal{Z}}, \quad \text { with } \quad \zeta_{0}=3.6 .
$$

where $\mathcal{Z} \equiv \sqrt{-\left.\frac{1}{2 \pi} \frac{d^{2} g}{d n^{2}}\right|_{n^{*}}}$ is the Zeldovich factor for unary condensation. A similar condition is found by Refs. [55, 56]: $n_{0}-n^{*} \gg(\sqrt{\pi} \mathcal{Z})^{-1} \Rightarrow \zeta_{0} \gg 1$. This condition is based on the reduction of the diffusion flux instead of the diffusion term and for $\epsilon=10^{-2}$ leads to $\zeta_{0}=7$, see Appendix C.

\subsection{Phase Path Analysis}

In Ref. [20] the so-called Phase Path Analysis (PPA) algorithm has been derived for an advected single-component vapor. In the present section we extend the method to a non-advected $N$-component vapor. Let $\Omega(t)$ represent a region in $\mathbf{n}$-space which is transported with the cluster growth rate. Application of the Reynolds transport theorem then yields

$$
\frac{d}{d t} \int_{\Omega(t)} d \mathbf{n}=\int_{\partial \Omega(t)} v \cdot \dot{\mathbf{n}} d s, \quad \Omega(0)=\Omega_{0},
$$

where $v$ is the outward unit normal on boundary $\partial \Omega(t)$ of $\Omega(t)$. Integration of the NGDE (6.5) over $\Omega(t)$ leads to

$$
\frac{d}{d t} \Phi(\Omega(t), t)=J_{\mathrm{s}}\left(\mathbf{n}_{0}(t)\right) H\left(\Omega(t), \mathbf{n}_{0}(t)\right),
$$

where $\Phi(\Omega(t), t)$ is the number of clusters in $\Omega(t)$ at time $t$

$$
\Phi(\Omega(t), t)=\int_{\Omega(t)} \rho(\mathbf{n}, t) d \mathbf{n},
$$

and

$$
H\left(\Omega, \mathbf{n}_{0}\right) \equiv \begin{cases}1 & \mathbf{n}_{0} \in \Omega \\ 0 & \mathbf{n}_{0} \notin \Omega .\end{cases}
$$

Eq. (6.40) can be integrated with respect to time from 0 to $t$

$$
\begin{aligned}
\Phi(\Omega(t), t) & =\Phi_{0}+\int_{0}^{t} J_{\mathrm{s}}\left(\mathbf{n}_{0}(\tau)\right) H\left(\Omega(\tau), \mathbf{n}_{0}(\tau)\right) d \tau, \\
\Phi_{0} & =\Phi\left(\Omega_{0}, 0\right) .
\end{aligned}
$$


To facilitate actual computation of this integral we introduce a characteristic surface $\overline{\mathbf{n}}\left(t ; \mathbf{n}_{0}(\tau), \tau\right)$ in $\mathbf{n}$-space defined by

$$
\frac{d}{d t} \overline{\mathbf{n}}=\dot{\mathbf{n}}(\overline{\mathbf{n}}, t), \quad \overline{\mathbf{n}}\left(\tau ; \mathbf{n}_{0}(\tau), \tau\right)=\mathbf{n}_{0}(\tau) .
$$

At fixed $t, \overline{\mathbf{n}}\left(t ; \mathbf{n}_{0}(\tau), \tau\right)$ represents a curve in the $\mathbf{n}$-plane that is parameterized by $\tau$, see Fig. 6.3 for a binary system, i.e. $N=2$.

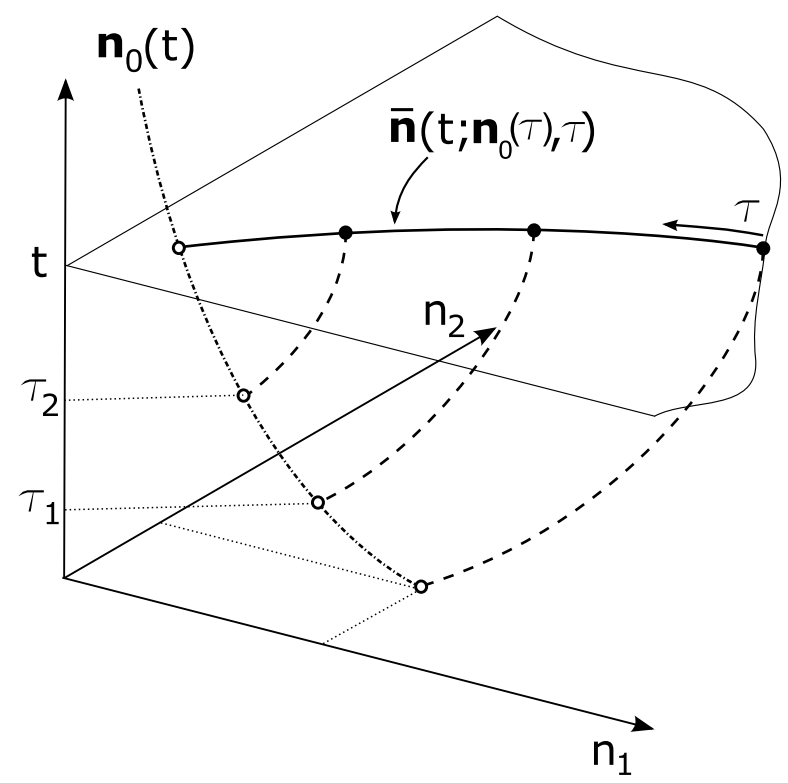

Figure 6.3: Characteristics (dashed lines) in two component $(\mathbf{n}, t)$-space originating from the source location $\mathbf{n}_{0}(t)$ (dash-dotted line with open circles). At fixed the distribution is given along a curve, $\overline{\mathbf{n}}\left(t ; \mathbf{n}_{0}(\tau), \tau\right)$.

Since both $\Omega$ and $\overline{\mathbf{n}}$ are transported at the cluster growth rate, it is observed that

$$
H\left(\Omega(\tau), \mathbf{n}_{0}(\tau)\right) \equiv H\left(\Omega(t), \overline{\mathbf{n}}\left(t ; \mathbf{n}_{0}(\tau), \tau\right)\right), \quad \forall t \geq \tau
$$

Eq. (6.46) is the $N$-component equivalent of Eq. (19) in Ref. [19]. With this expression, Eq. (6.43) can be rewritten in the computationally more convenient form

$$
\Phi(\Omega(t), t)=\Phi_{0}+\int_{0}^{t} J_{\mathrm{S}}\left(\mathbf{n}_{0}(\tau)\right) H\left(\Omega(t), \overline{\mathbf{n}}\left(t ; \mathbf{n}_{0}(\tau), \tau\right)\right) d \tau .
$$


For example, if the intersection of $\partial \Omega(t)$ and the curve $\overline{\mathbf{n}}\left(t ; \mathbf{n}_{0}(\tau), \tau\right)$ consists of two points $\overline{\mathbf{n}}\left(t ; \mathbf{n}_{0}\left(\tau_{1}\right), \tau_{1}\right)$ and $\overline{\mathbf{n}}\left(t ; \mathbf{n}_{0}\left(\tau_{2}\right), \tau_{2}\right), \tau_{2}>\tau_{1}$, then

$$
\Phi(\Omega(t), t)=\Phi_{0}+\int_{\tau_{1}(t)}^{\tau_{2}(t)} J_{\mathrm{s}}\left(\mathbf{n}_{0}(\tau)\right) d \tau .
$$

So the PPA algorithm for $N$-component systems comprises the tracing of the characteristics in (n,t)-space (Eq. (6.45)) and the calculation of the number of clusters per unit volume between the successive characteristics by means of Eq. (6.48). After this procedure we can reconstruct the curve $\overline{\mathbf{n}}$ providing the composition of the clusters. Furthermore, we define the corresponding distribution $\phi(s, t)$ along this curve as

$$
\phi(s, t) \equiv J_{\mathrm{s}}\left(\mathbf{n}_{0}(\tau)\right) /\left\|\frac{d \overline{\mathbf{n}}}{d \tau}\right\|, \quad s(\tau ; t) \equiv \int_{0}^{\tau}\left\|\frac{d \overline{\mathbf{n}}}{d \tau^{\prime}}\right\| d \tau^{\prime} .
$$

The curve length $s$ is defined for convenience since it scales with the total cluster size. We note that the $s$-derivative is well-defined, whereas the derivative to one of the constituents may not; a projection of the curve $\overline{\mathbf{n}}$ on the $n_{k}$-axis can lead to a self-intersection of the curve, resulting in a non-unique value for the derivative.

It is noted that direct comparison between the NBD solution and the NGDE solution is not possible since each of these distributions is of a different type. The NBD solution is defined in the entire $\mathbf{n}$-space, whereas the NGDE solution is a continuous but quasi-1D distribution. We therefore compare the distributions resulting from the NGDE with those from the NBD equations indirectly by evaluating their integral properties in the supercritical size domain $\Omega^{*}$ given by Eq. (3.17) in the form of the total cluster number density $\Phi$ and the superficial liquid mass density $l_{k}$. The total cluster number density is calculated using

$$
\begin{aligned}
\Phi^{\mathrm{NBD}}(t) & =\sum_{\mathbf{n} \in \Omega^{*}} \rho_{\mathbf{n}}(t), \\
\Phi^{\mathrm{NGDE}}(t) & =\int_{0}^{s(t ; t)} \phi(s, t) d s,
\end{aligned}
$$

Besides the total cluster number density, the superficial liquid mass density of component $k$ can be determined

$$
\begin{aligned}
l_{k}^{\mathrm{NBD}}(t) & =m_{k} \sum_{\mathbf{n} \in \Omega^{*}} n_{k} \rho_{\mathbf{n}}(t), \\
l_{k}^{\mathrm{NGDE}}(t) & =m_{k} \int_{0}^{s(t ; t)} \bar{n}_{k}(s) \phi(s, t) d s,
\end{aligned}
$$

for $k=1,2, \ldots, N$, where $m_{k}$ is the molecular mass of component $k$. 


\subsection{Test case definition}

We define two test nucleation pulse experiments of alcohol mixtures, e.g. see Ref. [71]. The first case considers a binary mixture and the results of the Binary General Dynamic Equation (BGDE) are compared to the corresponding results of the formally exact Binary Becker-Döring (BBD) equations. The second test case is a quinary mixture and serves as a demonstration of the efficiency and capabilities of the PPA algorithm.

\subsubsection{Binary nucleation pulse experiment}

We define a test case based on a typical nucleation pulse experiment for a binary condensing mixture of ethanol $(k=1)$, hexanol $(k=2)$ and an inert carrier gas (argon), e.g. see Ref. [71]. The carrier gas does not influence the nucleation behavior of the condensing components and serves as a heat sink during the condensation process. The test case consists of a stepwise constant pressure-temperature profile comprising a region with high vapor phase activities $a_{k} \equiv y_{k} p / p_{s, k}(T)$, in which $y_{k}$ and $p_{s, k}(T)$ are the vapor molar fraction and the saturation pressure of component $k$, respectively. In this region of high vapor phase activity, nucleation and growth take place and is referred to as region I or pulse region. The pulse region is succeeded by a longer time period with low vapor phase activities, in which nucleation is negligible (region II). The external conditions during the experiment are presented in Table 6.1. The pulse duration is $50 \mu \mathrm{s}$. The vapor is assumed non-depletable with mole fractions $\mathbf{y}=\left(1.117 \cdot 10^{-2}, 2.31 \cdot 10^{-4}\right)$. The mixture of ethanol and hexanol can be regarded as ideal and the pure component properties are listed in Appendix B.

\begin{tabular}{lcc}
\hline Parameter & $\begin{array}{c}\text { Region I } \\
(t \leq 50 \mu \mathrm{s})\end{array}$ & $\begin{array}{c}\text { Region II } \\
(50 \mu \mathrm{s}<t<300 \mu \mathrm{s})\end{array}$ \\
\hline$p[\mathrm{kPa}]$ & 66.76 & 74.00 \\
$T[\mathrm{~K}]$ & 260.0 & 270.5 \\
$\mathbf{a}$ & $(1.50,9.00)$ & $(0.72,2.86)$ \\
$\mathbf{n}^{*}$ & $(17,18)^{\mathrm{T}}$ & $(68,104)^{\mathrm{T}}$ \\
$\mathbf{n}_{0}$ & $(36.7,30.0)^{\mathrm{T}}$ & $(106.1,148.1)^{\mathrm{T}}$ \\
$\log _{10} J_{\mathrm{S}}\left[\mathrm{m}^{-3} \mathrm{~s}^{-1}\right]$ & 17.5 & -10.6 \\
\hline
\end{tabular}

TABLE 6.1: Nucleation pulse test case conditions for binary ethanol-hexanol mixture with vapor composition $\mathbf{y}=\left(1.117 \cdot 10^{-2}, 2.31 \cdot 10^{-4}\right)$ and $\zeta_{0}=3.6$.

\subsubsection{Quinary nucleation pulse experiment}

The quinary mixture contains ethanol $(k=1)$, propanol $(k=2)$, butanol $(k=3)$, pentanol $(k=4)$, hexanol $(k=5)$ and argon as a carrier gas. The vapor mole fractions 
of the alcohol mixture are: $\mathbf{y}=\left(7.446 \cdot 10^{-3}, 1.818 \cdot 10^{-3}, 7.786 \cdot 10^{-4}, 2.344\right.$. $10^{-4}, 1.026 \cdot 10^{-4}$ ). The nucleation pulse conditions are given in Table 6.2. The alcohol mixture can be regarded as ideal and the properties are listed in Appendix B.

\begin{tabular}{lcc}
\hline Parameter & $\begin{array}{c}\text { Region } I \\
(t \leq 50 \mu \mathrm{s})\end{array}$ & $\begin{array}{c}\text { Region II } \\
(50 \mu \mathrm{s}<t<300 \mu \mathrm{s})\end{array}$ \\
\hline$p[\mathrm{kPa}]$ & 66.76 & 74.00 \\
$T[\mathrm{~K}]$ & 260.0 & 270.5 \\
$\mathbf{a}$ & $(0.8,0.96,1.2,1.6,3.2)$ & $(0.39,0.4,0.49,0.58,1.02)$ \\
$\mathbf{n}^{*}$ & $(11,9,6,6,10)^{\mathrm{T}}$ & $(58,46,41,44,64)^{\mathrm{T}}$ \\
$\mathbf{n}_{0}$ & $(28.4,23.3,16.0,13.0,15.0)^{\mathrm{T}}$ & $(101.5,81.7,73.8,72.9,89.0)^{\mathrm{T}}$ \\
$\log _{10} J_{\mathrm{s}}\left[\mathrm{m}^{-3} \mathrm{~s}^{-1}\right]$ & 17.5 & -19.5 \\
\hline
\end{tabular}

TABLE 6.2: Nucleation pulse test case conditions for quinary alcohol mixture with composition $\mathbf{y}=\left(7.446 \cdot 10^{-3}, 1.818 \cdot 10^{-3}, 7.786 \cdot 10^{-4}, 2.344 \cdot 10^{-4}, 1.026 \cdot 10^{-4}\right)$ and $\zeta_{0}=3.6$.

\subsubsection{Computational method}

The BBD equations are solved by means of a sectional approximation in $\mathbf{n}$-space, extensively described in Ref. [65]. This method groups the clusters into bins and for this test case a resolution of $700 \times 700$ bins is used. The time integration is performed using the Heun predictor-corrector method [70] with a time step size of $10^{-10} s$. The partial vapor densities are prescribed at the origin of the $\mathbf{n}$-space, i.e. $\rho_{\mathbf{e}_{1}}=\rho_{v, 1}$ and $\rho_{\mathbf{e}_{2}}=\rho_{v, 2}$.

The characteristics in the PPA algorithm (Eq. (6.45)) and the integral in Eq. (6.48) are evaluated using second-order Heun time integration [70]. The number of characteristics is coupled to the time step size, as illustrated in Fig. 6.3. The reconstruction of $\phi(s, t)$ in Eq. (6.49) is carried out by means of a forward finite-difference method. The BGDE is solved using different values of $\zeta_{0}=[2,3.6,5]$ in Eq. (6.37) demonstrating the sensitivity of the method for the choice of the value of $\zeta_{0}$.

\subsection{Results and discussion}

The solution of the BBD equations has been obtained by parallel computation on 32 processors, requiring in a simulation time of approximately 10 days. In contrast, the solution of the BGDE with the PPA algorithm has been obtained in less than a minute on a single processor. Also the simulation of the quinary mixture using the NGDE is performed in order of minutes on a single processor. 
The computational time of the PPA method scales linearly with the number of components since the number of evaluations per phase path increases linearly with $N$. The NBD equations, however, require an additional grid dimension per component leading to an exponential increase of the computational effort with increasing $N$.

\subsubsection{Binary mixture}

The solution of the BGDE is compared with the solution of the formally exact BBD equations. Comparison are the composition and size of the clusters, the total cluster number density (Eq. (6.50)) and the superficial liquid mass density of the components (Eq. (6.51)).

Fig. 6.4 shows the comparison between the results of BBD equations and those of the BGDE for the cluster composition. The results are plotted at several time instants covering both regions of the nucleation pulse experiment. Note that the contours of the binary size distribution from the BBD equations are plotted on log-scale, indicating that the distribution has a strong maximum near the curve $\overline{\mathbf{n}}$ obtained from the BGDE (solid line). This means that the highest number density of a certain cluster size has a composition which agrees with the composition of that cluster size calculated by the BGDE. During the pulse (region I, Fig. 6.4a-c) the clusters are generated near the origin of the $\mathbf{n}$-space. After the pulse (region II, Fig. 6.4d-f) the distribution is transported in $\mathbf{n}$-space indicating that the nucleation has quenched, leaving cluster growth as the prevailing process.

Fig. 6.5 shows $\phi(s)$ in $\mathbf{n}$-space at several time instants, indicating the composition and the distribution of the binary clusters. We observe the typical decrease of the distribution with increasing size in the pulse region $(t=25 \mu \mathrm{s})$. Also, the sharp front at the large size part of the distribution is obtained similar to the distribution obtained in the single component case $[45,63,66]$. The typical decrease of the distribution with time is also observed in Fig. 6.5. This decrease is due to the stretching of the distribution, which is caused by the monotonically increasing growth rate with cluster size, i.e. $\frac{d \dot{n}_{k}}{d n_{k}}>0, \forall n_{k} \in \overline{\mathbf{n}}$.

Fig. 6.6 shows the comparison between the result of BBD equations and that of the BGDE in terms of the total cluster number density as function of time. We observe that the result from the BBD equations exhibits a certain relaxation time [82] $(\sim 2 \mu s)$, whereas the BGDE is based on the steady state nucleation rate. The instantaneous presence of the source in the supercritical size region results in a higher value of $\Phi$. For most nucleation pulse experiments the time of the pulse (region I) is much longer than the relaxation time of the BBD equations, resulting in a relatively smaller difference in $\Phi$ obtained from BBD and BGDE. However, due to excessive simulation times for solving the BBD equations an accurate numerical experiment with a pulse duration of the order of milliseconds is not feasible. The instantaneous decrease of $\Phi$ after the pulse is caused by the instantaneous increase of $\mathbf{n}^{*}$ resulting in a part of the cluster size distribution to become subcritical and to evaporate. The choice of $\zeta_{0}$ in 
Eq. (6.37) determines the magnitude of this decrease in $\Phi$. If $\zeta_{0}$ is decreased, a larger portion of the size distribution will evaporate after the nucleation pulse, as indicated in Fig. 6.6.

Fig. 6.7a shows the comparison of data for the superficial liquid mass density of ethanol obtained from the solution of the BBD equations and the one obtained from the BGDE. The activity of ethanol during the pulse is larger than unity, increasing $l_{1}$. After the pulse, the activity decreases resulting in the partial evaporation of ethanol molecules from the clusters. Fig. 6.7b shows the comparison of data for the superficial liquid mass density of hexanol obtained from the solution of the BBD equations and from the BGDE. The results of the BGDE for $l_{1}$ and $l_{2}$ correspond reasonably well with the BBD solution for various $\zeta_{0}$ values. We note that no unique value of $\zeta_{0}$ can be chosen such that all integral properties are in good agreement.

\subsubsection{Quinary mixture}

The results obtained by the PPA algorithm of the quinary mixture are presented in this section. Due to the excessively long computational times of the NBD equations the NGDE solution cannot be validated against the exact solution of the NBD equations. For the location of the source term we take $\zeta_{0}=3.6$. The time dependent total cluster number density $\Phi$ is depicted in Fig. 6.8(a). Similar to the binary case depicted in Fig. 6.6, the typical instantaneous decrease of $\Phi$ immediately following the pulse is observed. Fig. 6.8(b) gives the superficial liquid mass densities for all components as a function of time. The behavior of the components differs in the pulse and growth region. This is clarified in Fig. 6.8(c), where the liquid mass fraction $x_{k}=l_{k} / \sum_{i} l_{i}$ is shown. The composition of the clusters change rapidly at the onset of the nucleation pulse and during the pulse the mass fractions tend to converge to a steady state value. Following the pulse the conditions change and the ethanol evaporates leading to a new steady state composition. The choice of the nucleation pulse conditions determines the composition of the clusters at the end of the nucleation pulse experiment, i.e. at $t=300 \mu s$.

Fig. 6.9 shows the size dependence of the distribution $\phi$ and the liquid mass fractions $x_{k}$ at (a) $t=25 \mu \mathrm{s}$, (b) $t=100 \mu \mathrm{s}$ and (c) $t=300 \mu \mathrm{s}$. The PPA algorithm provides all properties, e.g. $\Phi(s), \mathbf{n}(s)$, as a function of the curve length $s$. The thick solid line in Fig. 6.9(a)-(c) indicates the size distribution $\phi(s)$ and shows similar behavior as in the binary case. In the initial stage of the nucleation pulse (Fig. 6.9(a)) the size distribution decreases strongly with increasing size until the front of the distribution is reached. The composition of the clusters changes with $s$ in the pulse region. Small clusters have a composition approximately equal to the composition at the source point $\mathbf{n}_{0}$. As the clusters grow the liquid mass fractions converges to the steady state values corresponding to the compositions found in the time dependent solution in Fig. 6.8(c). In the growth region the influence of the pulse region is apparently not important any more and the composition is almost size independent. 


\subsection{Conclusions}

Application of the PPA algorithm to the NGDE with $N=2$ reduces the computational time with a factor of $10^{5}$ compared to the computational time required for the solution method for the NBD equations. This extreme reduction of computing time of the NGDE relative to that of the NBD equations renders the method based on the NGDE feasible for the use in the design and analysis of nucleation pulse experiments, providing valuable information on the cluster composition and cluster size distribution. The method is suitable for any time dependent cluster formation process as long as the relaxation time of the formation process is smaller than the typical timescale of the experiment.

The results in Fig. 6.6 and 6.7 show that the choice of the starting value $\zeta_{0}$ influences the results. The value of $\zeta_{0}$ can be chosen by matching $\Phi^{\mathrm{NGDE}}$ with $\Phi^{\mathrm{NBD}}$, but this will deteriorate the agreement between $l_{k}^{\mathrm{NBD}}$ and $l_{k}^{\mathrm{NGDE}}$. The origin of the deviations is in the assumption of a steady state nucleation flux, as explained in Ref. [64] for single component condensation. Incorporating the time lag in the nucleation flux is expected to yield better results.

Application of the PPA algorithm to the NGDE with $N=5$ demonstrates the potential of the method. The simulation is performed in the order of minutes on a single processor and provides the approximate size distribution and the composition of the clusters. Unfortunately a comparison with the solution of the NBD equations cannot be made for $N=5$ due to the excessive computational effort that would be required.

In the present chapter we have demonstrated the validity of the model for vaporliquid transition, but the procedure is of general validity for any $\mathrm{N}$-component cluster formation process that exhibits a saddle point in $g(\mathbf{n})$. Moreover, the model could be extended to a $g(\mathbf{n})$ surface with two saddle points as has been observed in Ref. [84]. The right-hand side of Eq. (6.5) could be adjusted by adding a second Dirac $\delta$ function with a newly defined second source location. 


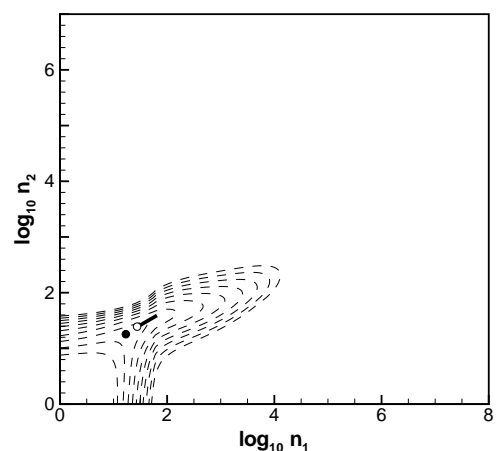

(a) $t=5 \mu \mathrm{s}$

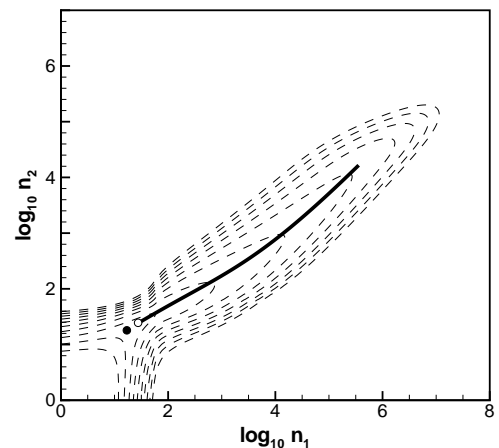

(c) $t=50 \mu \mathrm{s}$

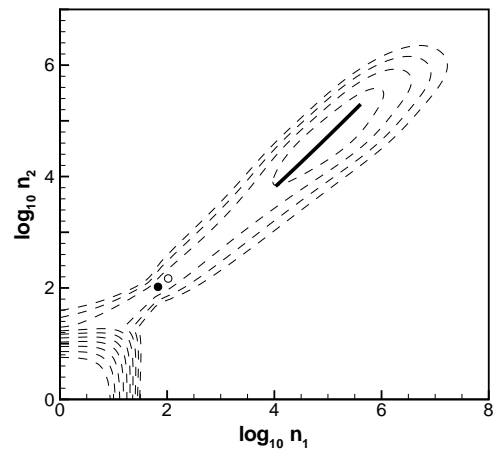

(e) $t=200 \mu \mathrm{s}$

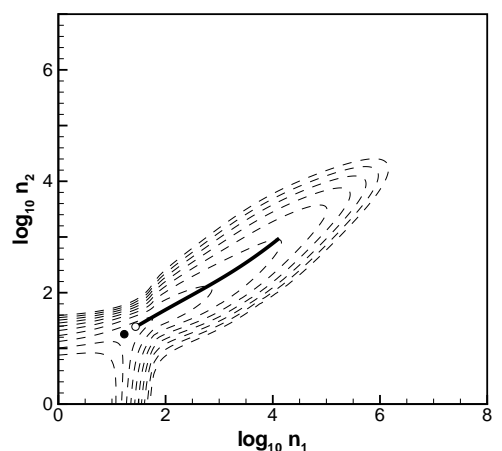

(b) $t=25 \mu \mathrm{s}$

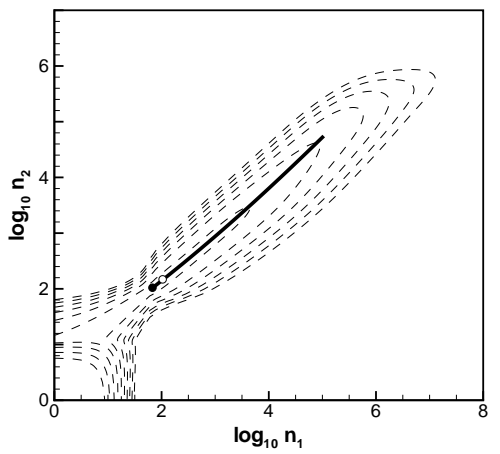

(d) $t=100 \mu \mathrm{s}$

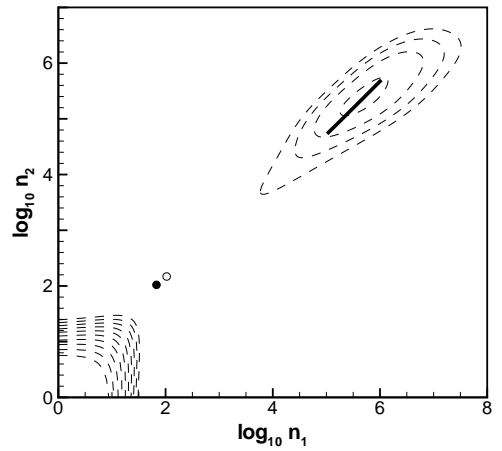

(f) $t=300 \mu \mathrm{s}$

FIGURE 6.4: Comparison of the cluster composition obtained from the BBD equations (dashed contour lines) and $\overline{\mathbf{n}}\left(t ; \mathbf{n}_{0}(\tau), \tau\right)$ (solid line) in the pulse region at (a) $t=5 \mu \mathrm{s}$, (b) $t=25 \mu \mathrm{s}$, (c) $t=50 \mu \mathrm{s}$ and in the growth region at (d) $t=100 \mu \mathrm{s}$, (e) $t=200 \mu \mathrm{s}$, (f) $t=300 \mu \mathrm{s}$ for $\zeta=3.6$ and conditions given in Table 6.1. The contour lines correspond to $\log _{10}\left(\rho_{\mathbf{n}}\right)$ from -5 to 15 with increments of 2 ; solid circle indicates $\mathbf{n}^{*}$ and open circle indicates $\mathbf{n}_{0}$. 


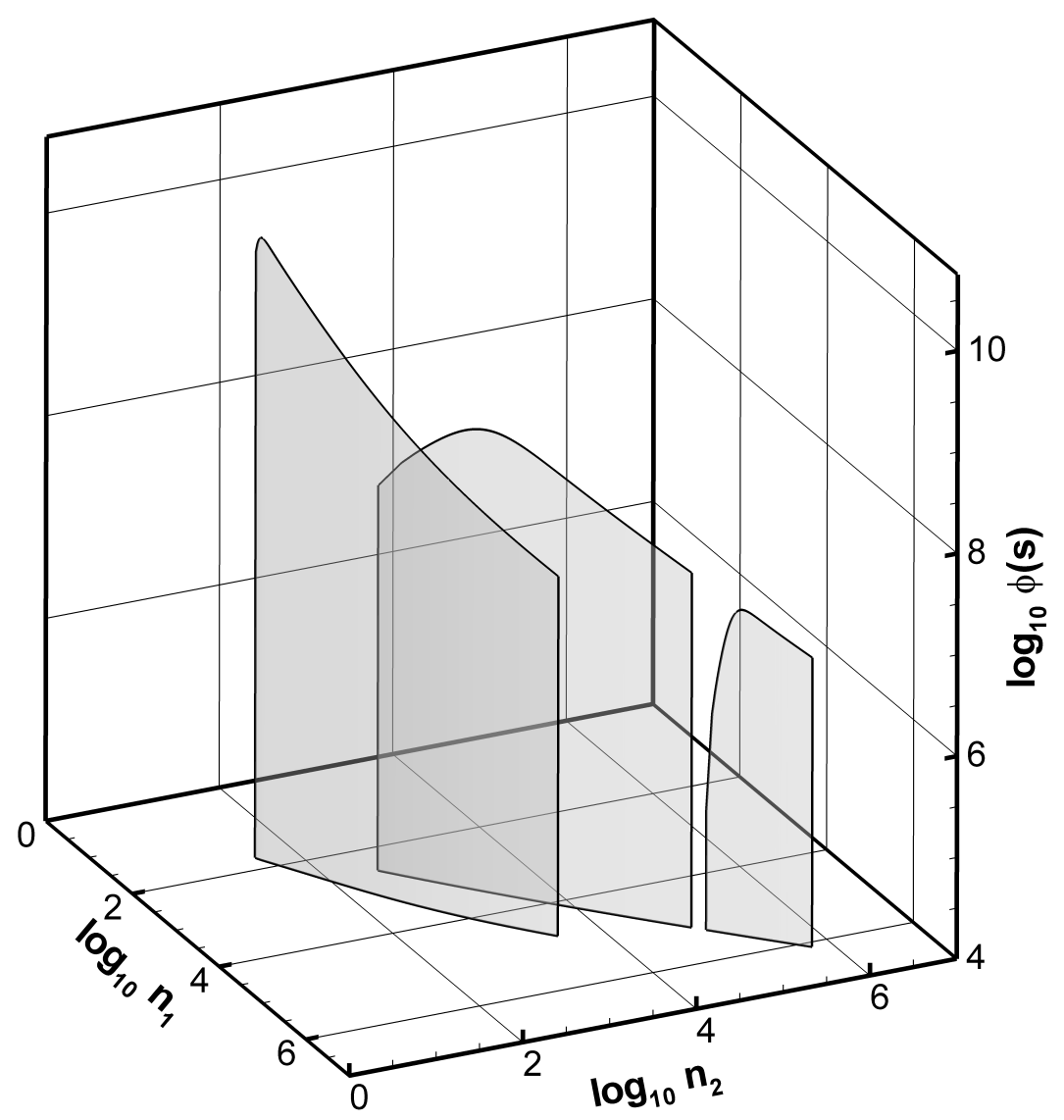

Figure 6.5: Distributions $\phi(s, t)$ in $\mathbf{n}$-space at $t=25 \mu \mathrm{s}$ in the pulse region and at $t=100 \mu \mathrm{s}$ and $t=300 \mu \mathrm{s}$ in the growth region for $\zeta=3.6$ and conditions given in Table 6.1 


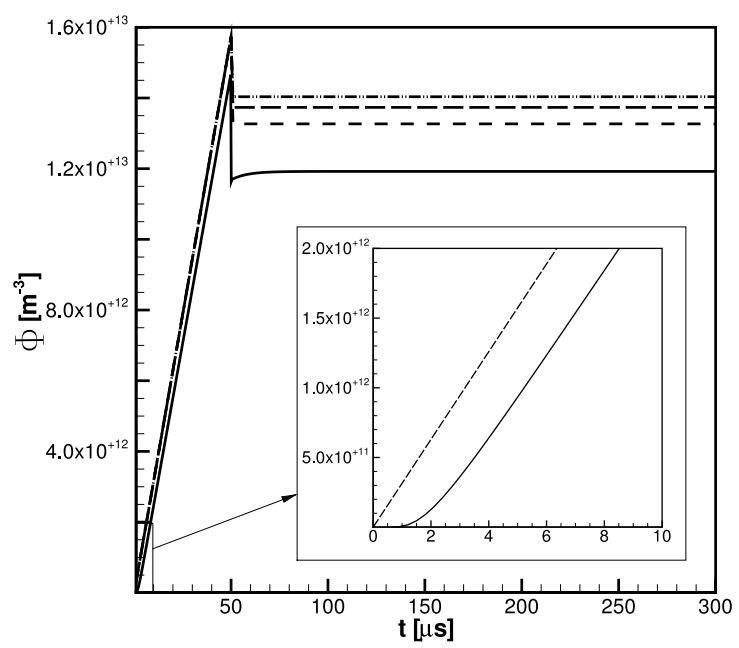

FIGURE 6.6: Comparison of the total cluster number density $\Phi^{\mathrm{BBD}}$ (solid line) and $\Phi^{\mathrm{BGDE}}$ for $\zeta_{0}=2$ (dashed line), $\zeta_{0}=3.6$ (long dashed line) and $\zeta_{0}=5$ (dash dotted line) as function of time for binary mixture nucleation pulse test case.

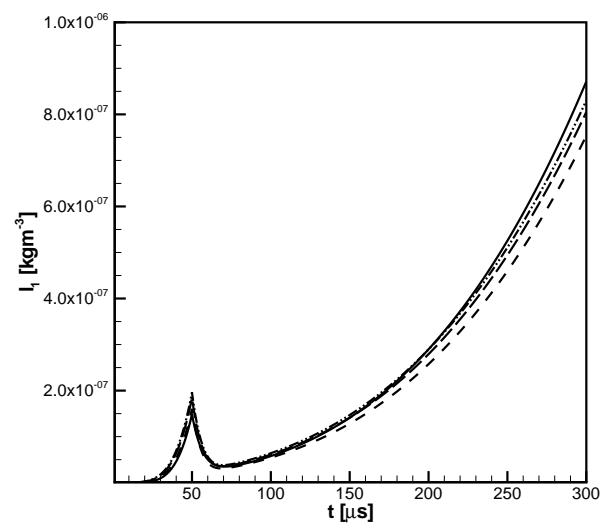

(a) $k=1$ : ethanol

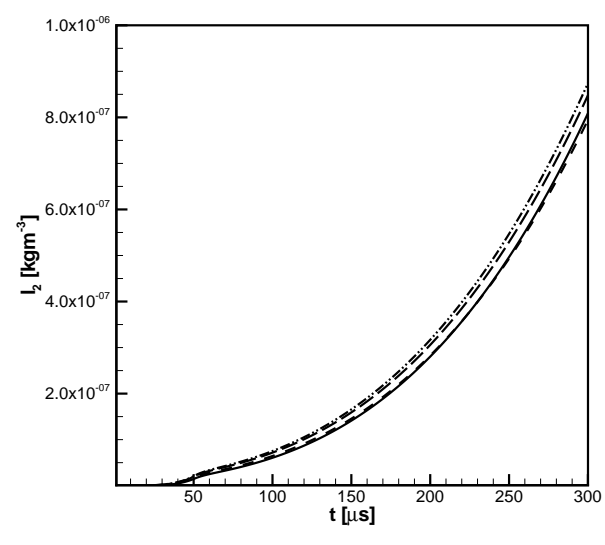

(b) $k=2$ : hexanol

FIGURE 6.7: Comparison of the $k$-component superficial liquid mass density ${ }_{k}^{\text {BDD }}$ (solid line) and $l_{k}^{\mathrm{BDE}}$ for $\zeta_{0}=2$ (dashed line), $\zeta_{0}=3.6$ (long dashed line) and $\zeta_{0}=5$ (dash dotted line) as function of time for the nucleation pulse test case, (a) ethanol $(k=1)$ and $(b)$ hexanol $(k=2)$. 


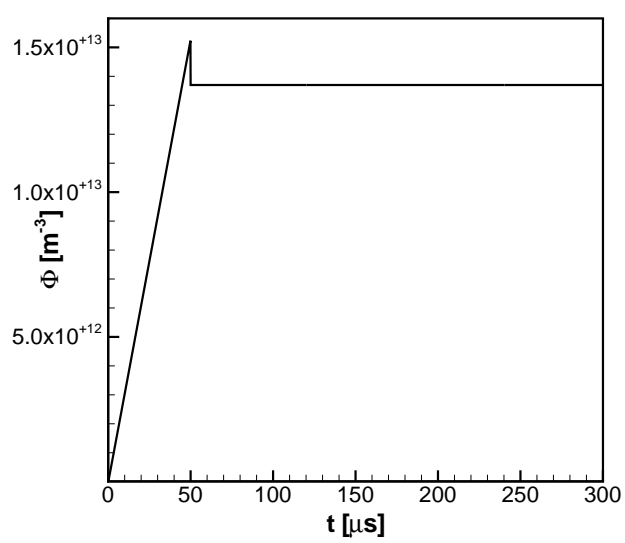

(a) total number of clusters

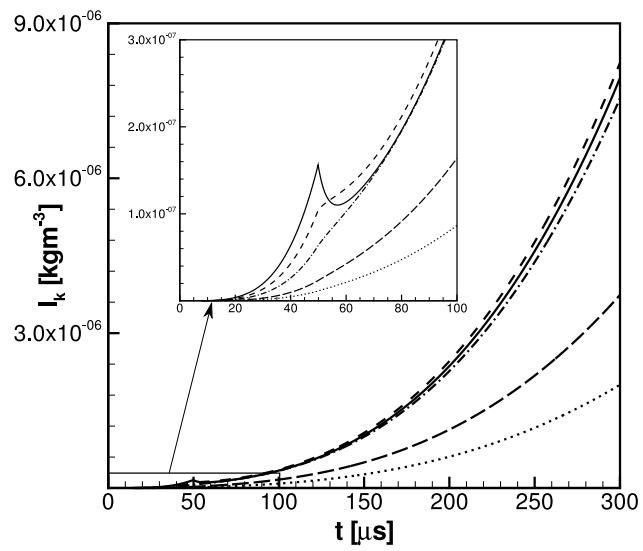

(b) superficial mass density

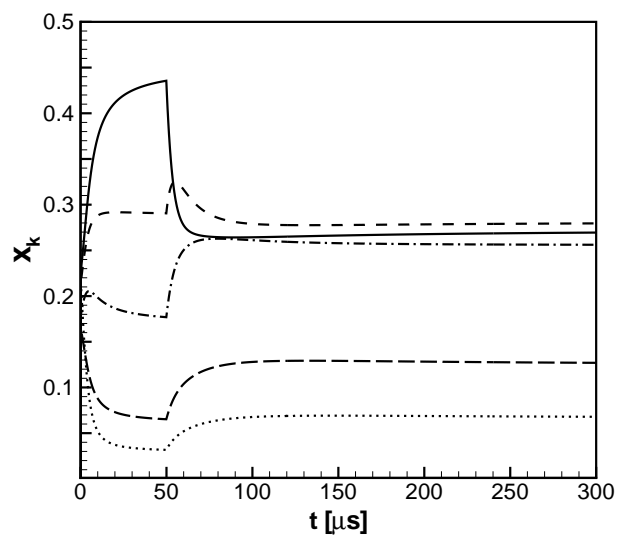

(c) liquid mass fraction

Figure 6.8: Time dependent solution of the NGDE $(N=5)$ for the mixture of ethanol (solid), propanol (short-dashed), butanol (dash-dotted), pentanol (long-dashed) and hexanol (dotted): (a) the total number of clusters $\Phi,(b)$ the superficial mass densities $l_{k}$ and $(c)$ the liquid mass fractions $x_{k}$. The conditions in the pulse region: $\mathbf{n}^{*}=(11,9,6,6,10)^{\mathrm{T}}, \mathbf{n}_{0}=(28.4,23.3,16.0,13.0,15.0)^{\mathrm{T}}, g\left(\mathbf{n}^{*}\right)=41.7$; in the growth region: $\mathbf{n}^{*}=(58,46,41,44,64)^{\mathrm{T}}, \mathbf{n}_{0}=(101.5,81.7,73.8,72.9,89.0)^{\mathrm{T}}$, $g\left(\mathbf{n}^{*}\right)=130.2$. 


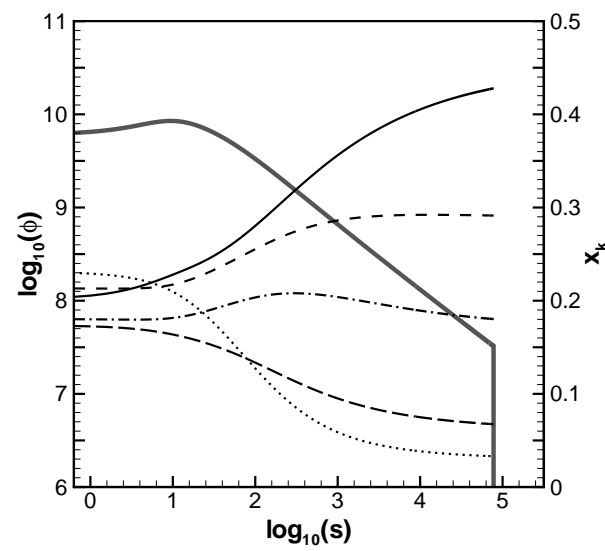

(a) $t=25 \mu \mathrm{s}$

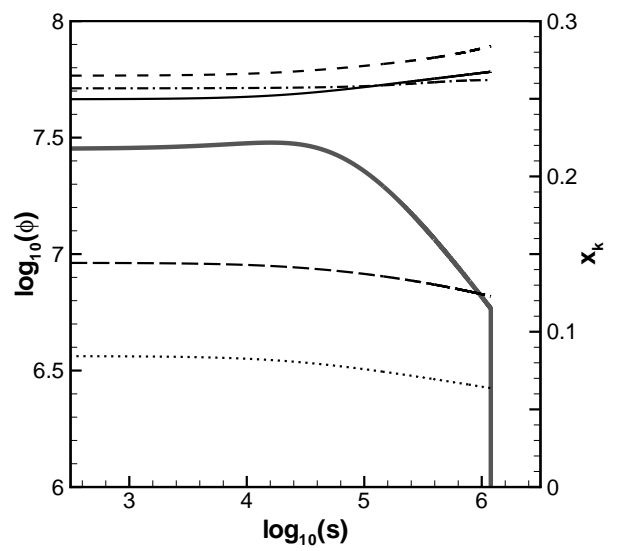

(b) $t=100 \mu \mathrm{s}$

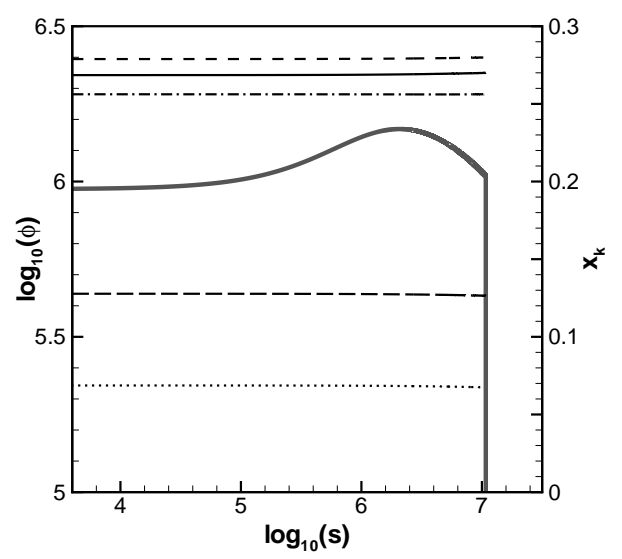

(c) $t=300 \mu \mathrm{s}$

Figure 6.9: Size dependent solution of the $\operatorname{NGDE}(N=5)$ for the mixture of ethanol (solid), propanol (short-dashed), butanol (dash-dotted), pentanol (long-dashed) and hexanol (dotted): at (a) $t=25 \mu \mathrm{s}$, (b) $t=100 \mu \mathrm{s}$ and (c) $t=300 \mu \mathrm{s}$. The thick solid line is the size distribution $\phi$ and the thin lines are the liquid mass fractions $x_{k}$ of the constituents. 



\section{Conclusions AND RECOMMENDATIONS}

\subsection{Conclusions}

In Chapter 2 a robust numerical solution method has been developed for the calculation of three-phase equilibria for real mixtures. The method is based on NewtonRaphson iteration and a proper initialization scheme has been presented based on pressure extrapolation. The commonly used initialization schemes are insufficiently accurate for high pressure calculations. The derivatives needed in the iterative scheme and the initialization scheme are calculated analytically for the components which are accurately described by the SRK equation of state. The results for a ternary threephase mixture are calculated and give useful insight in the multiphase behavior of real gases.

In Chapter 4 a multigrid algorithm has been derived for the numerical solution of the $N$-component Becker-Döring (NBD) equations. The standard multigrid coarsening techniques fail for the $N$-component nucleation problem, but the developed geometrical coarsening scheme does work. The proposed coarsening conserves the fluxes and yields equivalent sets of equations on the coarsened grids. The method can be used for an arbitrary number of components and reduces the computational time by a factor of 10 compared to the computing time required for general iterative solvers. The ternary nucleation problem has been investigated by solving the time evolution of the cluster size distribution in the initial stages of the formation process. The method is applicable to small clusters and can aid in the development of theories for steady state nucleation.

In Chapter 5 an approximate model has been derived from the unary Fokker-Planck Equation (FPE). The model assumes a steady state cluster size distribution in the subcritical cluster size region which leads to an analytic expression for the diffusion flux in the FPE. The resulting model is termed the Stationary Diffusion Flux (SDF) model and is applicable to all cluster sizes. Comparison of the results of the SDF with the results of the $\mathrm{BD}$ equations shows an excellent agreement for the size distribution up to the monomers.

In Chapter 6 the efficient Phase Path Analysis algorithm has been introduced for 
the $N$-component General Dynamic Equation (NGDE). The location of the source term in the NGDE has been derived rigorously and depends on the free energy of cluster formation and the $\mathrm{N}$-component gas kinetics. The algorithm can be applied to supercritical clusters and is capable of calculating the approximate size distribution for large clusters. The method has been validated for the case of a nucleation pulse experiment for a binary mixture and comparing the size, composition and integral properties of the approximate distribution with the outcome of the formally exact NBD equations. The method reduces the computational effort by a factor of $10^{5}$ for the binary mixture.

\subsection{Recommendations}

Several recommendations are proposed for possible future research:

1. The method for initialization in Chapter 2 can also be extended for the temperature, see Eq. (2.22). The resulting expressions for extrapolation in pressure and temperature can be exploited such that one of the variables in the solution vector remains constant. Preferably the pressure and temperature increments can be chosen such that the vapor phase fraction is constant. One then obtains a scheme which solves the variable vector by tracing the variables along isocontours of the vapor phase fraction and omits the use of an iterative scheme. Therefore this method is potentially very efficient while maintaining high resolution equilibrium pressure-temperature data.

2. The multigrid method in Chapter 4 is a first step in the development of efficient multigrid algorithms for the present type of problems. At the moment it is limited to the small cluster size region. This is due to the geometrical construction of the coarse grid levels. To extend the method to the region of larger cluster size one could use a bin structure for the original NBD equations on the target grid as developed for binary mixtures in Ref. [65]. More recently, Algebraic MultiGrid (AMG) algorithms have been developed, see e.g. Ref. [75]. These methods are not restricted to the geometrical construction of the grid levels. The coarsening is based on the shape of the operator $L$ in the sense that strongly coupled elements in the solution vector $\mathbf{u}$ are maintained on the coarse grid. This coarsening is expected to be more efficient since it automatically locates the regions of interest and disregards the less important regions which are solved in the geometrical approach.

3. The SDF model has been derived for single component condensation. The model can be extended to $N$-component mixtures given an appropriate choice of a curvilinear coordinate system in the subcritical cluster size region. In the 
large cluster size region the stationary diffusion flux in the direction perpendicular to the growth rate vector cannot be neglected.

4. The Phase Path Analysis algorithm for the NGDE in Chapter 6 is expected to be a suitable method for validation of $N$-component nucleation experiments. Due to the reduced computational time, the method can be used as a design tool for experimental devices (e.g. nozzle geometries [73]) based on the integral properties of the $N$-component size distribution. For these fast expanding nozzle flows the coupling between the condensation and the fluid dynamics equations need to be established similarly as has been achieved in Ref. [65]. Also the extension to incorporate the depletion of the vapor is mandatory to simulate the behavior in these condensing flows accurately. 



\section{REFERENCES}

[1] M. Abramowitz and I. A. Stegun, Handbook of Mathematical Functions (Dover, New York, 1972).

[2] F. F. Abraham, "Multistate Kinetics in Nonsteady-State Nucleation: A Numerical Solution", J. Chem. Phys. 51, 1632 (1969).

[3] J. M. Ball, J. Carr and O. Penrose, "The Becker-Döring Cluster Equations: Basic Properties and Asymptotic Behaviour of Solutions", Commun. Math. Phys. 104, 657 (1986).

[4] R. Becker and W. Döring, "Kinetische Behandlung der Keimbildung in Übersättigten Dämpfern", Ann. Phys. 24, 719 (1935).

[5] A. Brandt, "Multi-Level Adaptive Solutions to Boundary-Value Problems", Math. Comp. 31, 333 (1977).

[6] A. Brandt, Multigrid Techniques: 1984 Guide with Applications to Fluid Dynamics (GMD, Bonn, 1984).

[7] A. Brandt, J. Bernholc and K. Binder, Multiscale computational methods in chemistry and physics, volume 177 of NATO Science Series III: Computer and systems sciences (IOS press, Amsterdam, 2001).

[8] D. Brus, V. Ždímal and J. Smolík, "Homogeneous nucleation rate measurements in supersaturated water vapor", J. Chem. Phys. 129, 174501 (2008).

[9] R. Courant, K. Friedrichs and H. Lewy, "Über die partiellen Differenzengleigungen der mathematischen Physik", Math. Ann., 100, 3274 (1928).

[10] C. F. Delale, G. H. Schnerr and J. Zierep, "Asymptotic solution of transonic nozzle flows with homogeneous condensation. 1. Subcritical flows", Phys. Fluids A 5, 2969 (1993). 
[11] D. B. Duncan and A. R. Soheili, "Approximating the Becker-Döring cluster equations", Appl. Num. Math. 37, 1 (2001).

[12] M. C. Facchini, M. Mircea, S. Fuzzi and R. J. Charlson, "Cloud albedo enhancement by surface-active organic solutes in growing droplets", Nature 401, 257 (1999).

[13] S. P. Fisenko and G. Wilemski, "Kinetics of binary nucleation of vapors in size and composition space", Phys. Rev. E 70, 056119 (2004).

[14] J. Frenkel, Kinetic Theory of Liquids (Dover, New York, 1955).

[15] A. I. Gaman, M. Kulmala, H. Vehkamäki, I. Napari, M. Mircea, M. C. Facchini and A. Laaksonen, "Binary homogeneous nucleation in watersuccinic acid and waterglutaric acid systems", J. Chem. Phys. 120, 282 (2004).

[16] S. P. Glazenborg, Multi-level techniques for the solution of the Kinetic Equations in condensation flows, MSc. thesis, University of Twente, 2010.

[17] F. Gelbard and J. H. Seinfeld, "The General Dynamic Equation for Aerosols: Theory and Application to Aerosol Formation and Growth", J. Colloid Interface Sci. 68, 363 (1979).

[18] L. Gránásy and P. F. James, "Nucleation and growth in cluster dynamics: A quantitative test of the classical kinetic approach", J. Chem. Phys. 113, 9810 (2000).

[19] R. Hagmeijer, "Equivalence of two different integral representations of droplet distribution moments in condensing flow", Phys. Fluids 16, 176 (2004).

[20] R. Hagmeijer, R. H. A. IJzermans and F. Put, "Solution of the general dynamic equation along approximate fluid trajectories generated by the method of moments", Phys. Fluids 17, 056101 (2005).

[21] J. O. Hirschfelder, "Kinetics of homogeneous nucleation on many-component systems", J. Chem. Phys. 61, 2690 (1974).

[22] V. Holten and M. E. H. van Dongen, "Comparison between solutions of the general dynamic equation and the kinetic equation for nucleation and droplet growth", J. Chem. Phys. 130, 014102 (2009).

[23] D. Kashchiev, Nucleation: basic theory with applications (ButterworthHeinemann, Oxford, 2000).

[24] V. I. Kalikmanov, "Mean-field kinetic nucleation theory", J. Chem. Phys. 124, 124505 (2006). 
[25] V. I. Kalikmanov, "Binary nucleation beyond capillarity approximation", Phys. Rev. E 81, 050601 (R) (2010).

[26] D-M. Kim and P. D. Iedema, "Modeling of branching density and branching distribution in low-density polyethylene polymerization", Chem. Eng. Sci. 63, 2035 (2008).

[27] K.-J. Kim, M.-J. Kim, J.-M. Lee, S.-H. Kim, H.-S. Kim and B.-S. Park, ”Experimental solubility and density for 3-nitro-1,2,4-triazol-5-one $+C_{1}$ to $C_{7} 1$ alkanols”, Fluid Phase Equil. 146, 261 (1998).

[28] J. J. Jasper, "The Surface Tension of Pure Liquid Compounds", J. Phys. Chem. Ref. Data 1, 841 (1972).

[29] P. Lancaster and M. Tismenetsky, The Theory of Matrices (Academic Press, San Diego, 1985).

[30] B. van Leer, "Towards the Ultimate Conservative Difference Scheme. V. A Second-Order Sequel to Godunovs Method", J. Comp. Phys. 32, 101 (1979).

[31] J.-S. Li and G. Wilemski, "Temperature Dependence of a Kelvin Model for Binary Nucleation", J. Phys. Chem. B 105, 11778 (2001).

[32] E. M. Lifshitz and L. P. Pitaevskii, Physical Kinetics (Butterworth-Heinemann, Oxford, 2006).

[33] C. C. M. Luijten, K. J. Bosschaart and M. E. H. van Dongen, "High pressure nucleation in water/nitrogen systems", J. Chem. Phys. 106, 8116 (1997).

[34] R. B. McClurg and R. C. Flagan, "Critical Comparison of Droplet Models in Homogeneous Nucleation Theory”, J. Colloid Interface Sci. 201, 194 (1998).

[35] R. McGraw, "Two-dimensional kinetics of binary nucleation in sulfuric acidwater mixtures", J. Chem. Phys. 102, 2098 (1995).

[36] M. L. Michelsen, "The isothermal flash problem. Part I: Stability Analysis", Fluid Phase Equil. 9, 1 (1982).

[37] M. L. Michelsen, "The isothermal flash problem. Part II: Phase-Split Calculation", Fluid Phase Equil. 9, 21 (1982).

[38] O. Penrose, "The Becker-Döring Equations at Large Times and Their Connection with the LSW Theory of Coarsening", J. Stat. Phys. 89, 305 (1997).

[39] R. H. Perry and D. W. Green and J. O. Maloney, The Chemical Engineers' Handbook (McGraw-Hill, New York, 1997). 
[40] B. E. Poling and J. M. Prausnitz and J. P. O'Connell, The Properties of Gases and Liquids (McGraw-Hill, New York, 2001).

[41] S. E. Pratsinis, "Flame aerosol synthesis of ceramic powders", Prog. Energy Combust. Sci. 24, 197 (1998).

[42] J. M. Prausnitz, R. N. Lichtenthaler and E. Gomez de Azevedo, Molecular thermodynamics of fluid-phase equilibrium (Prentice-Hall, New Jersey, 1986).

[43] W. H. Press, S. A. Teukolsky, W. T. Vetterling and B. P. Flannery, Numerical Recipes in Fortran 77: The Art of Scientific Computing (Cambridge University Press, New York, 2007).

[44] F. Put, Numerical Simulation of Condensation in Transonic Flows, PhD. thesis, University of Twente, 2003; P. H. Kelleners, An Edge-based Finite Volume Method for Inviscid Compressible Flow with Condensation PhD. thesis, University of Twente, 2007.

[45] D. S. van Putten and V. I. Kalikmanov, "Efficient approach to nucleation and growth dynamics: Stationary diffusion flux model", J. Chem. Phys. 130, 164508 (2009).

[46] D. S. van Putten, R. S. R. Sidin and R. Hagmeijer, "Efficient approximation of the cluster size distribution in binary condensation", J. Chem. Phys. 132, 184511 (2010).

[47] D. S. van Putten, S.P. Glazenborg, R. Hagmeijer and C. H. Venner, "A multigrid method for $N$-component nucleation", J. Chem. Phys. 135, 014114 (2011).

[48] V. Raghavan and M. Cohen, "A nucleation model for martensitic transformations in iron-base alloys", Acta Metall. 20, 333 (1972).

[49] H. Reiss, "The Kinetics of Phase Transitions in Binary Systems", J. Chem. Phys. 18, 840 (1950).

[50] H. Risken, The Fokker-Planck Equation: Methods of Solution and Applications (Springer-Verlag, Berlin, 1989).

[51] J. W. P. Schmelzer, "On the determination of the kinetic pre-factor in classical nucleation theory", J. Non-Cryst. Solids 354, 269 (2008).

[52] J. H. Seinfeld, Atmospheric Chemistry and Physics of Air Pollution (John Wiley and Sons, New York, 1986).

[53] G. Shi and J. H. Seinfeld, "Kinetics of binary nucleation: Multiple pathways and the approach to stationarity", J. Chem. Phys. 93, 9033 (1990). 
[54] V. A. Shneidman, "The kinetics of first-order phase transitions. Non-stationary many-parameter nucleation", Sov. Phys. JETP 64, 306 (1986).

[55] V. A. Shneidman, "Size distribution of new-phase particles during transient condensation of a supercooled gas", Sov. Phys. Tech. Phys. 32, 76 (1987).

[56] V. A. Shneidman, "Establishment of a steady-state nucleation regime. Theory and comparison with experimental data for glasses", Sov. Phys. Tech. Phys. 33, 1338 (1988).

[57] V. A. Shneidman, "Dynamics of an Ising ferromagnet at $T \leq T_{c}$ from the droplet model approach", Physica A 190, 145 (1992).

[58] V. A. Shneidman and W. C. Weinberg, "Induction time in transient nucleation theory", J. Chem. Phys. 97, 3621 (1992).

[59] V. A. Shneidman and W. C. Weinberg, "Transient nucleation induction time from the birth-death equations", J. Chem. Phys. 97, 3629 (1992).

[60] V. A. Shneidman and M. C. Weinberg, "The effects of transient nucleation and size-dependent growth rate on phase transformation kinetics", J. Non-Cryst. Solids 130, 89 (1993).

[61] V. A. Shneidman and M. C. Weinberg, "Crystallization of rapidly heated amorphous solids", J. Non-Cryst. Solids 194, 145 (1996).

[62] V. A. Shneidman, "Transient nucleation distributions and fluxes at intermediate times and sizes", J. Chem. Phys. 115, 8141 (2001).

[63] V. A. Shneidman, "Universal Distributions Generated in a Nucleation Pulse", Phys. Rev. Lett. 101, 205702 (2008).

[64] V. A. Shneidman, "Transient nucleation with a monotonically changing barrier", J. Chem. Phys. 132, 047101 (2010).

[65] R. S. R. Sidin, "Droplet Size Distribution in Condensing Flow”, Ph.D. thesis, University of Twente, 2009.

[66] R. S. R. Sidin, R. Hagmeijer and U. Sachs, "Evaluation of master equations for the droplet size distribution in condensing flow", Phys. Fluids 21, 073303 (2009).

[67] V. V. Slezov and J. Schmelzer, "Kinetics of formation and growth of a new phase with a definite stoichiometric composition", J. Phys. Chem. Solids 55, 243 (1994). 
[68] V. V. Slezov, Kinetics of First-order Phase Transitions (Wiley-VCH, Weinheim, 2009).

[69] J. M. Smith, H. C. van Ness and M. M. Abbott, Introduction to Chemical Engineering Thermodynamics (McGraw-Hill, New York, 2005).

[70] J. Stoer and R. Bulirsch, Introduction to Numerical Analysis (second edition, Springer-Verlag, New York, 1993).

[71] R. Strey and Y. Viisanen, "Measurement of the molecular content of binary nuclei. Use of the nucleation rate surface for ethanol-hexanol", J. Chem. Phys. 99, 4693 (1993).

[72] R. Strey, P. E. Wagner and Y. Viisanen, "The Problem of Measuring Homogeneous Nucleation Rates and the Molecular Contents of Nuclei: Progress in the Form of Nucleation Pulse Measurements", J. Phys. Chem. 98, 7748 (1994).

[73] S. Tanimura, B. E. Wyslouzil, M. S. Zahniser, J. H. Shorter, D. D. Nelson and J. B. McManus, "Tunable diode laser absorption spectroscopy study of $\mathrm{CH} 3 \mathrm{CH} 2 \mathrm{OD} / \mathrm{D} 2 \mathrm{O}$ binary condensation in a supersonic Laval nozzle", J. Chem. Phys. 127, 034305 (2007).

[74] H. Trinkaus, "Theory of the nucleation of multicomponent precipitates", Phys. Rev. B 27, 7372 (1983).

[75] U. Trottenberg, C. Oosterlee and A. Schüller, Multigrid (Elsevier, Amsterdam, 2001).

[76] C. H. Venner and A. A. Lubrecht, Multilevel Methods in Lubrication (Elsevier, Amsterdam, 2007).

[77] Y. Viisanen, M. Kulmala and A. Laaksonen, "Experiments on gasliquid nucleation of sulfuric acid and water", J. Chem. Phys. 107, 920 (1997).

[78] G. Wilemski and B. E. Wyslouzil, "Binary nucleation kinetics. I. Self-consistent size distribution", J. Chem. Phys. 103, 1127 (1995).

[79] J. Wölk and R. Strey, "Homogeneous nucleation of $\mathrm{H}_{2} \mathrm{O}$ and $\mathrm{D}_{2} \mathrm{O}$ in comparison: the isotope effect", J. Phys. Chem. B 105, 11683 (2001).

[80] D. T. Wu, "General approach to barrier crossing in multicomponent nucleation", J. Chem. Phys. 99, 1990 (1993).

[81] B. E. Wyslouzil and G. Wilemski, "Binary nucleation kinetics. II. Numerical solution of the birth-death equations", J. Chem. Phys. 103, 1137 (1995). 
[82] B. E. Wyslouzil and G. Wilemski, "Binary nucleation kinetics. III. Transient behavior and time lags", J. Chem. Phys. 105, 1090 (1996).

[83] B. E. Wyslouzil, C. H. Heath, J. L. Cheung and G. Wilemski, "Binary condensation in a supersonic nozzle", J. Chem. Phys. 113, 7317 (2000).

[84] B. E. Wyslouzil and S. Chen, "Binary Nucleation Kinetics. 6. Partially Miscible Systems", J. Chem. Phys. 105, 11566 (2001).

[85] Ya. B. Zeldovich, "On the Theory of New Phase Formation. Cavitation", Acta Physicochim. URSS 18, 1 (1943). 



\section{EQUILIBRIUM THERMODYNAMICS FOR SRK EQUATION OF STATE}

In this appendix the expressions for the equilibrium calculations are derived in further extent. The derivation is applicable to both the liquid and vapor phase, so the superscript from Eq. (2.13) is dropped. The elaboration will be performed using the SRK equation of state, given by

$$
p=\frac{k_{\mathrm{B}} T}{v-b}-\frac{a(T)}{v(v+b)},
$$

where $a(T)$ and $b$ for a pure component are given by

$$
\begin{aligned}
a(T) & =\frac{0.42748 k_{\mathrm{B}}^{2} T_{c}^{2}}{p_{c}}\left[1+\left(0.480+1.574 \omega-0.176 \omega^{2}\right)\left(1-T_{r}^{\frac{1}{2}}\right)\right]^{2}, \\
b & =\frac{0.08664 k_{\mathrm{B}} T_{c}}{p_{c}} .
\end{aligned}
$$

\section{A.1 Fugacity coefficient and chemical potential}

The calculation of fugacity coefficient involves the compressibility factor $Z$, which is related to the pressure as

$$
Z \equiv \frac{p v}{k_{\mathrm{B}} T}=\frac{v}{v-b_{m}}-\frac{a_{m}}{k_{\mathrm{B}} T\left(v+b_{m}\right)} .
$$

For the evaluation of the integral in Eq. (2.13) the partial compressibility factor is required, which is defined as $\bar{Z}_{i} \equiv\left(\frac{\partial n Z}{\partial n_{i}}\right)_{T, V, n_{j \neq i}}$ with $n$ is the total number of molecules and $n_{i}$ is the number of molecules of component $i$. Elaboration for the SRK equation of state and rewriting in terms of the total volume $V=n v$, yields

$$
\bar{Z}_{i}=\frac{V}{V-b_{m} n}+\frac{n V \bar{b}_{i}}{\left(V-b_{m} n\right)^{2}}-\frac{n \bar{a}_{i}}{k_{\mathrm{B}} T\left(V+b_{m} n\right)}+\frac{a_{m} n^{2} \bar{b}_{i}}{k_{\mathrm{B}} T\left(V+b_{m} n\right)^{2}},
$$


where the derivatives are taken at constant temperature, volume and number of molecules of all other components. The partial parameters $\bar{a}_{i}$ and $\bar{b}_{i}$ can be calculated by using the averaging from Section 2.1.2

$$
\bar{a}_{i} \equiv \frac{1}{n}\left(\frac{\partial n^{2} a_{m}}{\partial n_{i}}\right)_{T, n_{j \neq i}}=2 \sqrt{a_{i}} \sum_{j=1}^{N} x_{j} \sqrt{a_{j}}\left(1-k_{i j}\right), \quad \bar{b}_{i} \equiv\left(\frac{\partial n b_{m}}{\partial n_{i}}\right)_{T, n_{j \neq i}}=b_{i} .
$$

It is noted that in Eq. (A.5) the binary interaction parameters, $l_{i j}$, are assumed to be equal to zero in agreement with Section 2.1.2. The integrand in Eq. (2.13) thus becomes

$$
\frac{\bar{Z}_{i}-1}{V}=\frac{1}{V-n b_{m}}-\frac{1}{V}+\frac{n \bar{b}_{i}}{\left(V-n b_{m}\right)^{2}}-\frac{n \bar{a}_{i}}{k_{\mathrm{B}} T V\left(V+n b_{m}\right)}+\frac{n^{2} a_{m} \bar{b}_{i}}{k_{\mathrm{B}} T V\left(V+n b_{m}\right)^{2}},
$$

where the molar volume is replaced by the total volume, i.e. $V=n v$. Integrating Eq. (A.6) and rewriting in terms of molecular volume, results in

$$
\begin{aligned}
\ln \phi_{i}= & -\ln \left(\frac{v-b_{m}}{v}\right)+\frac{\bar{a}_{i}}{k_{\mathrm{B}} T b_{m}} \ln \left(\frac{v}{v+b_{m}}\right)-\frac{a_{m} \bar{b}_{i}}{k_{\mathrm{B}} T b_{m}^{2}} \ln \left(\frac{v}{v+b_{m}}\right)-\ln (Z) \\
& +\frac{\bar{b}_{i}}{\left(v-b_{m}\right)}-\frac{a_{m} \bar{b}_{i}}{k_{\mathrm{B}} T v\left(v+b_{m}\right)} .
\end{aligned}
$$

The last two contributions reduce to $\frac{\bar{b}_{i}}{b}(Z-1)$ by using Eq. (A.3). In terms of the compressibility factor, the fugacity coefficient for the SRK equation of state becomes

$$
\ln \phi_{i}=-\ln (Z-B)+\frac{\bar{b}_{i}}{b_{m}}(Z-1)-\frac{A}{B}\left(\frac{\bar{a}_{i}}{a_{m}}-\frac{\bar{b}_{i}}{b_{m}}\right) \ln \left(\frac{Z+B}{Z}\right),
$$

where $A$ and $B$ are defined as

$$
A=\frac{a_{m} p}{k_{\mathrm{B}}^{2} T^{2}}, \quad \text { and } \quad B=\frac{b_{m} p}{k_{\mathrm{B}} T} .
$$

\section{A.2 Compressibility factor}

For the calculation of the fugacity coefficient, the compressibility factor is needed. Substitution the definition of $Z$ in Eq. (A.3) in the equation of state, results in

$$
Z^{3}-Z^{2}+\left(A-B-B^{2}\right) Z-A B=0 .
$$

This equation can be solved either analytically or numerically, where the parameters $A$ and $B$ are determined separately for the liquid and the vapor phase. To prevent numerical instabilities, an analytic solution procedure is used as proposed by Ref. [1]. The compressibility factor for the liquid and the vapor phase are given by the lowest and highest root of Eq. (A.10), respectively. 


\section{A.3 Elements of the Jacobian}

The majority of elements in the Jacobian in Eq. (2.19) are zero. Let $F_{k}$ denote the $k^{\text {th }}$ equation of the function vector, then the sub-matrices are given by

$$
\begin{array}{lllll}
\frac{\partial F_{1, i}}{\partial x_{j}^{c}}=-K_{i}^{c} \delta_{i j}-x_{i}^{c} \frac{\partial K_{i}^{c}}{\partial x_{j}^{c}}, & \frac{\partial F_{1, i}}{\partial x_{j}^{u}}=0, & \frac{\partial F_{1, i}}{\partial x_{j}^{j}}=\delta_{i j}-x_{i}^{c} \frac{\partial K_{i}^{c}}{\partial x_{j}^{v}}, & \frac{\partial F_{1, i}}{\partial \mathcal{V}}=0, & \frac{\partial F_{1, i}}{\partial C}=0, \\
\frac{\partial F_{2, i}}{\partial x_{j}^{c}}=0, & \frac{\partial F_{2, i}}{\partial x_{j}^{a}}=-K_{i}^{a} \delta_{i j}-x_{i}^{a} \frac{\partial K_{i}^{a}}{\partial x_{j}^{a}}, & \frac{\partial F_{2, i}}{\partial x_{j}^{v}}=\delta_{i j}-x_{i}^{a} \frac{\partial K_{i}^{a}}{\partial x_{j}^{v}}, & \frac{\partial F_{2, i}}{\partial \mathcal{V}}=0, & \frac{\partial F_{2, i}}{\partial C}=0, \\
\frac{\partial F_{3, i}}{\partial x_{j}^{c}}=-C \delta_{i j} & \frac{\partial F_{3, i}}{\partial x_{j}^{a}}=(\mathcal{V}+C-1) \delta_{i j}, & \frac{\partial F_{3, i}}{\partial x_{j}^{v}}=-\mathcal{V} \delta_{i j}, & \frac{\partial F_{3, i}}{\partial \mathcal{V}}=x_{i}^{a}-x_{i}^{v}, & \frac{\partial F_{3, i}}{\partial C}=x_{i}^{a}-x_{i}^{c}, \\
\frac{\partial F_{4}}{\partial x_{j}^{c}}=0, & \frac{\partial F_{4}}{\partial x_{j}^{a}}=1, & \frac{\partial F_{4}}{\partial x_{j}^{v}}=0, & \frac{\partial F_{4}}{\partial \mathcal{V}}=0, & \frac{\partial F_{4}}{\partial C}=0, \\
\frac{\partial F_{5}}{\partial x_{j}^{c}}=0, & \frac{\partial F_{5}}{\partial x_{j}^{a}}=0, & \frac{\partial F_{5}}{\partial x_{j}^{v}}=1, & \frac{\partial F_{5}}{\partial \mathcal{V}}=0, & \frac{\partial F_{5}}{\partial C}=0,
\end{array}
$$

where $i, j=1, \ldots, N$.

\section{A.4 Derivatives of $K_{i}^{\alpha}$}

For the equilibrium flash problem and initialization algorithm we need the derivatives of $K_{i}^{\alpha}$ with respect to pressure, temperature and phase fractions. The derivatives can be evaluated numerically by means of a finite difference method. For the hydrocarbon components, which are accurately described by the SRK equation of state, the derivatives can be evaluated analytically and written in the following convenient form

$$
\frac{\partial K_{i}^{c}}{\partial \vartheta}=\frac{d}{d \vartheta}\left(\frac{\phi_{i}^{c}}{\phi_{i}^{v}}\right)=K_{i}^{c}\left(\frac{d \ln \phi_{i}^{c}}{d \vartheta}-\frac{d \ln \phi_{i}^{v}}{d \vartheta}\right), \quad \text { with } \quad \vartheta=p, T, x_{j}^{\alpha} .
$$

where $\alpha=v, c$. The derivatives of the fugacity coefficient (Eq. (A.8)) with respect to $\vartheta$ is given by

$$
\begin{aligned}
\frac{\partial \ln \phi_{i}}{\partial \vartheta}= & -\frac{\frac{\partial Z}{\partial \vartheta}-\frac{\partial B}{\partial \vartheta}}{Z-B}+\frac{\bar{b}_{i}}{b_{m}}\left(\frac{\partial Z}{\partial \vartheta}-(Z-1) \frac{\partial \ln b_{m}}{\partial \vartheta}\right)-\frac{A}{B}\left\{\left[\left(\frac{\partial \ln A}{\partial \vartheta}-\frac{\partial \ln B}{\partial \vartheta}\right)\left(\frac{\bar{a}_{i}}{a_{m}}-\frac{\bar{b}_{i}}{b_{m}}\right)\right.\right. \\
& \left.+\frac{\bar{a}_{i}}{a_{m}}\left(\frac{\partial \ln \bar{a}_{i}}{\partial \vartheta}-\frac{\partial \ln a_{m}}{\partial \vartheta}\right)+\frac{\bar{b}_{i}}{b_{m}} \frac{\partial \ln b_{m}}{\partial \vartheta}\right] \ln \left(\frac{Z+B}{Z}\right) \\
& \left.+\left(\frac{\bar{a}_{i}}{a_{m}}-\frac{\bar{b}_{i}}{b_{m}}\right) \frac{B}{Z+B}\left(\frac{\partial \ln B}{\partial \vartheta}-\frac{\partial \ln Z}{\partial \vartheta}\right)\right\},
\end{aligned}
$$

where we dropped the superscript $\alpha$ for clarity. The complexity of this derivative depends on the variable and the equation of state considered. For Eq. (A.13) we 
require the derivative of $Z$ with respect to $\vartheta$

$$
\frac{\partial Z}{\partial \vartheta}=\frac{\frac{\partial A}{\partial \vartheta}(B-Z)+\frac{\partial B}{\partial \vartheta}(A+Z+2 B Z)}{3 Z^{2}-2 Z+A-B-B^{2}} .
$$

The derivatives of $A$ and $B$ with respect to pressure and fraction can be evaluated straightforwardly, see Eq. (A.5) and (A.9). The complexity of the derivatives with respect to temperature, however, depends on the equation of state and for SRK are given by

$$
\begin{aligned}
& \frac{\partial A}{\partial T}=A\left(\frac{\partial \ln a_{m}}{\partial T}-\frac{2}{T}\right), \\
& \frac{\partial B}{\partial T}=-\frac{B}{T} .
\end{aligned}
$$

Using the mixture rules for $a_{m}$ from Section 2.1.2 yields

$$
\begin{aligned}
\frac{\partial a_{m}}{\partial T} & =-\sum_{i=1}^{N} \sum_{j=1}^{N} x_{i} x_{j} \sqrt{a_{i} a_{j}}\left(1-k_{i j}\right) v_{i}, \\
\frac{\partial \bar{a}_{i}}{\partial T} & =-\sqrt{a_{i}} \sum_{j=1}^{N} x_{j} \sqrt{a_{j}}\left(1-k_{i j}\right)\left(v_{i}+v_{j}\right),
\end{aligned}
$$

where $v_{i}$ originates from the temperature dependence of $a_{i}$ in Table 2.1, i.e. $\frac{\partial a_{i}}{\partial T}=$ $-a_{i} v_{i}$, with

$$
v_{i} \equiv \frac{0.480+1.574 \omega_{i}-0.176 \omega_{i}^{2}}{\left[1+\left(0.480+1.574 \omega_{i}-0.176 \omega_{i}^{2}\right)\left(1-T_{r, i}^{\frac{1}{2}}\right)\right] \sqrt{T T_{c, i}}} .
$$

The derivatives with respect to the fractions $x_{j}$ are

$$
\begin{aligned}
\frac{\partial A}{\partial x_{j}} & =\frac{\bar{a}_{j} A}{a_{m}}, \\
\frac{\partial B}{\partial x_{j}} & =\frac{\bar{b}_{j} B}{b_{m}},
\end{aligned}
$$

with $\bar{a}_{j}$ and $\bar{b}_{j}$ given by Eq. (A.5). Finally, we evaluate the derivatives of $\bar{a}_{i}$ with respect to $x_{j}$

$$
\frac{\partial \bar{a}_{i}}{\partial x_{j}}=2 \sqrt{a_{i} a_{j}}\left(1-k_{i j}\right)
$$

which is phase independent. 


\section{THERMO-PHYSICAL PROPERTIES}

The thermo-physical properties of ethanol, propanol, butanol, pentanol and hexanol are taken from several sources. The saturation pressure ([Pa]) for the pure component follows from [40]

$$
p_{s, k}=p_{c, k} \exp \left(\frac{a_{k} \tau_{k}+b_{k} \tau_{k}^{1.5}+c_{k} \tau_{k}^{2.5}+d_{k} \tau_{k}^{5}}{T_{r, k}}\right)
$$

where $p_{c, k}$ and $T_{c, k}$ are the critical pressure and temperature, respectively, $\tau_{k} \equiv 1-T_{r, k}$ and $T_{r, k}=T / T_{c, k}$ is the reduced temperature. For ethanol $(k=1)$, propanol $(k=2)$, butanol $(k=3)$, pentanol $(k=4)$ and hexanol $(k=5)$ the parameters are

$$
\begin{array}{lll}
a_{1}=-8.68587, & a_{2}=-8.53706, & a_{3}=-8.40615, \\
b_{1}=1.17831, & b_{2}=1.96214, & b_{3}=2.2301, \\
c_{1}=-4.8762, & c_{2}=-7.6918, & c_{3}=-8.2486, \\
d_{1}=-1.588, & d_{2}=-2.945, & d_{3}=-0.711, \\
p_{c, 1}=6.13 \cdot 10^{6} \mathrm{~Pa}, & p_{c, 2}=5.17 \cdot 10^{6} \mathrm{~Pa}, & p_{c, 3}=4.42 \cdot 10^{6} \mathrm{~Pa}, \\
T_{c, 1}=513.92 \mathrm{~K}, & T_{c, 2}=536.78 \mathrm{~K}, & T_{c, 3}=563.05 \mathrm{~K} . \\
a_{4}=-8.98005, & a_{5}=-9.49034, & \\
b_{4}=3.91624, & b_{5}=5.13288, & \\
c_{4}=-9.9081, & c_{5}=-10.5817, & \\
d_{4}=-2.191, & d_{5}=-5.154, & \\
p_{c, 4}=3.91 \cdot 10^{6} \mathrm{~Pa}, & p_{c, 5}=3.47 \cdot 10^{6} \mathrm{~Pa}, & \\
T_{c, 4}=588.15 \mathrm{~K}, & T_{c, 5}=610.7 \mathrm{~K} . &
\end{array}
$$


The liquid density $\left(\left[\mathrm{kg} / \mathrm{m}^{3}\right]\right)$ from Ref. [27] for the component of the alcohol mixture are given by

$$
\begin{aligned}
& \rho_{l, 1}=1060.6-0.95929 T, \\
& \rho_{l, 2}=1050.1-0.84682 T, \\
& \rho_{l, 3}=1050.3-0.88124 T, \\
& \rho_{l, 4}=1049.8-0.79527 T, \\
& \rho_{l, 5}=1044.1-0.76716 T .
\end{aligned}
$$

The surface tension $([\mathrm{N} / \mathrm{m}])$ is taken from Ref. [28]

$$
\begin{aligned}
& \sigma_{1}=10^{-3}(24.05-0.0832(T-273.15)), \\
& \sigma_{2}=10^{-3}(25.26-0.0777(T-273.15)), \\
& \sigma_{3}=10^{-3}(27.18-0.0898(T-273.15)), \\
& \sigma_{4}=10^{-3}(27.54-0.0874(T-273.15)), \\
& \sigma_{5}=10^{-3}(26.44-0.0869(T-273.15)) .
\end{aligned}
$$




\section{$N$-COMPONENT DYNAMICS NEAR THE CRITICAL SIZE}

In this appendix we derive useful properties of the $N$-component cluster dynamics near the critical size $\mathbf{n}^{*}$. The growth rate vector and the integration of the nucleation flux vector are elaborated. We finalize this appendix with a discussion on the source term location of the NGDE.

\section{C.1 Growth rate vector}

The growth rate vector in $\mathbf{n}$-space is given by Eq. (6.4). Using Eq. (6.7), the growth rate in the vicinity of the saddle point can be written as

$$
\dot{\mathbf{n}}(\mathbf{n})=\mathrm{T}_{\mathbf{n}}^{-1} \Delta \mathbf{n}, \quad \text { with } \quad \mathrm{T}_{\mathbf{n}} \equiv-\frac{1}{2}\left(\mathrm{D}^{*} \mathrm{H}^{*}\right)^{-1} .
$$

The matrix $\mathrm{T}_{\mathbf{n}}$ is the Zeldovich time parameter matrix for $N$-component condensation in the n-coordinate system. Similarly, the general expression for the growth rate vector $\dot{\xi}=\mathrm{A}^{-1} \dot{\mathbf{n}}$ near $\boldsymbol{\xi}^{*}$ with A from Eq. (6.12), becomes

$$
\dot{\boldsymbol{\xi}}(\boldsymbol{\xi})=\mathrm{T}_{\xi}^{-1} \boldsymbol{\xi}, \quad \text { with } \quad \mathrm{T}_{\xi} \equiv-\frac{1}{2} \operatorname{diag}\left(\lambda_{1}^{-1}, \lambda_{2}^{-1}, \ldots, \lambda_{N}^{-1}\right),
$$

where $\mathrm{T}_{\xi}$ is the Zeldovich time parameter matrix in the $\boldsymbol{\xi}$-coordinate system. We note that Eq. (C.2) can be obtained directly by the simultaneous diagonalizing transformation (Eq. (6.14)) of the Zeldovich time parameter matrix in Eq. (C.1).

Given that $\lambda_{1}<0$ and $\lambda_{k}>0$ for $k=2, \ldots, N$ the growth rate in $\xi_{1}$-direction is unstable around the critical size $\xi^{*}$, i.e. $\dot{\xi}_{1}>0$ for $\xi_{1}>0$ and $\dot{\xi}_{1}<0$ for $\xi_{1}<0$. This implies that in all stable directions, where $\lambda_{k}>0(k=2, \ldots, N)$, the growth rate forces the clusters to converge to the $\xi_{1}$-axis.

\section{C.2 Nucleation flux integration}

In this section we derive the integration domain of Eq. (6.26) in the transformed coordinate system [80]. In the $\mathbf{n}$-space we define the vector $\nabla_{\mathbf{n}} \xi_{1}$ normal to the surface 
$S_{0}$ given by

$$
S_{0}=\left\{\mathbf{n} \in \mathbb{R}^{N} \mid \mathbf{n}=A \boldsymbol{\xi}+\mathbf{n}^{*}, \xi_{1}=\xi_{1}^{0}\right\} .
$$

A schematic representation is given in Fig. C.1. The $N$-component nucleation rate is

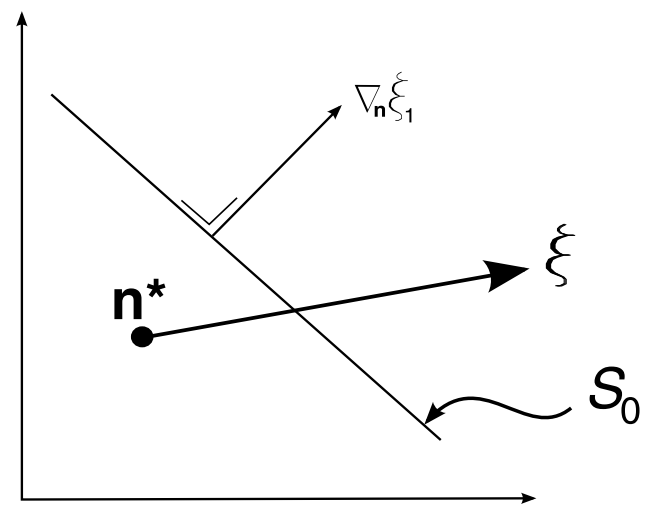

n

FiguRE C.1: Representation of the integration surface $S_{0}$ and the vectors $\boldsymbol{\xi}$ and $\nabla_{\mathbf{n}} \xi_{1}$ in $\mathbf{n}$-space.

determined by integration of the nucleation flux vector over the hyper-surface $S_{0}$

$$
J_{\mathrm{S}}=\int_{S_{0}} \mathbf{J}_{\mathbf{n}} \cdot \boldsymbol{v} d S, \quad \text { with } \quad \boldsymbol{v}=\frac{\nabla_{\mathbf{n}} \xi_{1}}{\left|\nabla_{\mathbf{n}} \xi_{1}\right|},
$$

the unit normal vector to the surface $S_{0}$.

We define the volume $V$ as the integral over the transformed $\xi$-space

$$
V\left(\xi_{1}^{0}, \Sigma\right)=\int_{0}^{\xi_{1}^{0}}\left\{\int_{\Sigma} \operatorname{det}(A) d \xi_{2} d \xi_{3} \ldots d \xi_{N}\right\} d \xi_{1},
$$

with $\Sigma$ an arbitrary subset of $\mathbb{R}^{N-1}$ and $V$ is the map of $\left[0, \xi_{1}^{0}\right] \times \Sigma$. We require that the change of the volume $V$ in $\xi_{1}$-direction can be written in both coordinate systems as

$$
\begin{aligned}
\frac{\partial V}{\partial \xi_{1}^{0}} & =\int_{S} \frac{\partial \mathbf{n}}{\partial \xi_{1}} \cdot v d S, \\
\frac{\partial V}{\partial \xi_{1}^{0}} & =\int_{\Sigma} \operatorname{det}(A) d \xi_{2} d \xi_{3} \ldots d \xi_{N} .
\end{aligned}
$$


where $S$ is the map of $\Sigma$. Since $\Sigma$ is arbitrary this leads to

$$
d S=\frac{\operatorname{det}(A)}{\frac{\partial \mathbf{n}}{\partial \xi_{1}} \cdot v} d \xi_{2} d \xi_{3} \ldots d \xi_{N} .
$$

We now discuss the transformed nucleation flux. In $\mathbf{n}$-space the flux is given by

$$
\mathbf{J}_{\mathbf{n}}=-\rho^{\mathrm{eq}} \mathrm{D} \nabla_{\mathbf{n}} \gamma_{\mathrm{s}}=-\rho^{\mathrm{eq}} \mathrm{DA}^{-\mathrm{T}} \nabla_{\xi} \gamma_{\mathrm{s}},
$$

whereas the transformed flux is

$$
\mathbf{J}_{\xi}=-\rho^{\mathrm{eq}} \nabla_{\xi} \gamma_{\mathrm{s}},
$$

leading to

$$
\mathbf{J}_{\mathbf{n}}=\mathrm{DA}^{-\mathrm{T}} \mathbf{J}_{\boldsymbol{\xi}} .
$$

Due to the simultaneous diagonalization we have $\mathrm{A}^{-1} \mathrm{DA}^{-\mathrm{T}}=\mathrm{I}$ and thus $\mathrm{DA}^{-\mathrm{T}}=\mathrm{A}$ yielding

$$
\mathbf{J}_{\mathbf{n}}=\mathrm{A} \mathbf{J}_{\xi} .
$$

Using $\mathbf{J}_{\xi}=\left(J_{\xi_{1}}, 0, \ldots, 0\right)^{\mathrm{T}}$ in Eq. (C.12), the integrand in Eq. (C.4) can be rewritten as

$$
\mathbf{J}_{\mathbf{n}} \cdot \boldsymbol{v}=\frac{\partial \mathbf{n}}{\partial \xi_{1}} \cdot \boldsymbol{v} J_{\xi_{1}} .
$$

Hence using Eq. (C.13) and (C.8) in Eq. (C.4), the steady state nucleation rate becomes

$$
J_{\mathrm{S}}=\int_{\mathbb{R}^{N-1}} J_{\xi_{1}} \operatorname{det}(A) d \xi_{2} d \xi_{3} \ldots d \xi_{N} .
$$

\section{C.3 Source point location}

The value of $\zeta_{0}$ in Eq. (6.37) can be derived from the $\zeta$-dependence $\left(\zeta \equiv \sqrt{\pi} \xi_{1}\right)$ of the steady state diffusion flux. Using the steady state cluster size distribution $\overline{\rho_{\mathrm{S}}}$ from Eq. (6.29) we can write for the scaled diffusion and drift flux in $\xi_{1}$-direction, respectively,

$$
\begin{aligned}
-J_{\mathrm{s}}^{-1} \frac{d \bar{\rho}_{\mathrm{s}}}{d \xi_{1}} & =\left[1-\sqrt{\pi} \zeta \exp \left(\zeta^{2}\right) \operatorname{erfc}(\zeta)\right] \equiv \Upsilon(\zeta), \\
J_{\mathrm{s}}^{-1} \dot{\xi_{1}} \overline{\rho_{\mathrm{s}}} & =\sqrt{\pi} \zeta \exp \left(\zeta^{2}\right) \operatorname{erfc}(\zeta) \equiv 1-\Upsilon(\zeta) .
\end{aligned}
$$

The scaling with the steady state nucleation rate is applied such that the rhs functions are normalized. Fig. C. 2 depicts the rapid decay of the function $\Upsilon(\zeta)$ and therefore the rapid decay of the normalized diffusion flux. The function $\Psi(\zeta)$ in Fig. C.2 is 
based on the decrease of the diffusion term given in Eq. (6.35). Both functions are related as

$$
\frac{d \Upsilon}{d \zeta}=\sqrt{\pi} \Psi .
$$

The vertical lines Fig. C.2 indicate two conditions for $\zeta_{0}$ in Eq. (6.37) given by

$$
\begin{aligned}
& \Psi\left(\zeta_{0}\right)=\epsilon \Psi(0), \rightarrow \zeta_{0}=3.6 \\
& \Upsilon\left(\zeta_{0}\right)=\epsilon \Upsilon(0), \rightarrow \zeta_{0}=7.0
\end{aligned}
$$

where $\epsilon=10^{-2}$ is a measure for the decay of the functions with respect to the value at the critical size, i.e. $\zeta=0$. The sensitivity of the NGDE for the choice of $\zeta_{0}$ is discussed in Section 6.5.1.

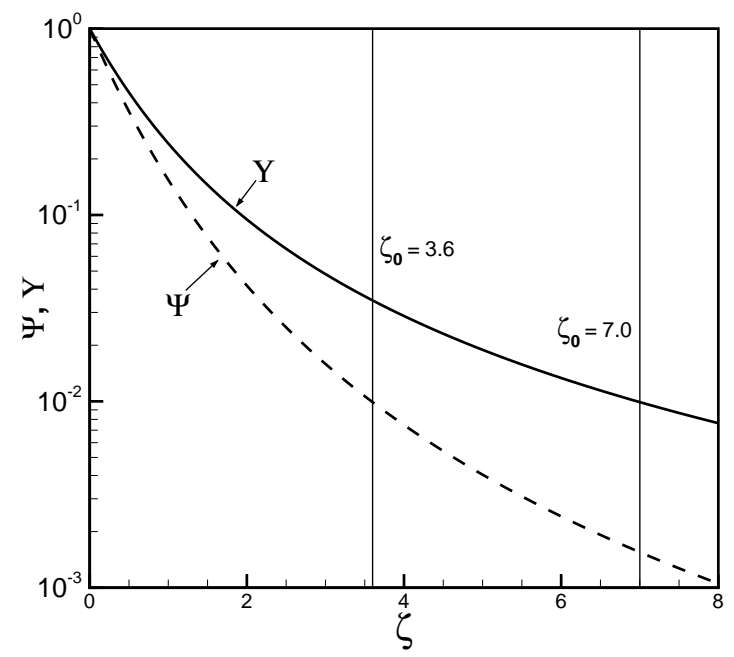

Figure C.2: The functions $\Upsilon$ (solid) and $\Psi$ (dashed) as a function of $\zeta$. The thin vertical lines indicate $\zeta_{0}=7.0$ (diffusion flux based) and $\zeta_{0}=3.6$ (diffusion term based). 


\section{SAMENVATTING}

Het proefschrift beschrijft efficiënte methoden ontwikkeld voor $N$-componenten condensatie processen. Deze methoden zijn verkregen door enerzijds het gebuik van efficiente numerieke methoden en anderzijds door de vereenvoudiging van de wiskundigefysische beschrijving. De modellen en bijbehorende algoritmes verschillen in het vermogen om details van de fysica van het condensatie proces te beschrijven en in de benodigde rekentijd.

Het proefschrift presenteert evenwichts thermodynamica van reële gassen zowel als de ontwikkeling van een robuuste numerieke oplosmethode gebaseerd op NewtonRaphson iteratie. De Jacobiaan is analytisch uitgewerkt. Het initialisatie schema voor deze oplosmethode maakt gebruik van extrapolatie van de oplos vector in termen van de druk. Resultaten worden gepresenteerd voor een drie-fase ternair reëel gas mengsel.

Een multigrid methode is ontwikkeld voor de verhoging van de efficiëntie van impliciete methodes voor het numeriek oplossen van de $N$-componenten Becker-Döring (NBD) vergelijkingen. Deze methode gebruikt een geometrische multigrid algoritme voor een willekeurig aantal grid levels. Het multigrid algoritme lost de volledige set van NBD vergelijkingen 10 keer sneller op dan de gangbare iteratieve schema's. Desondanks is de methode alleen toepasbaar in het regime van kleine clusters, dit vanwege de beperkte beschikbare rekencapaciteit. Echter, de verkregen tijdsafhankelijke oplossing van de NBD vergelijkingen geeft nuttige fysische inzichten in de initiële fase van het nucleatie proces.

Voor unaire condensatie is het Stationary Diffusion Flux (SDF) model afgeleid. Dit model kent geen beperking ten aanzien van cluster grootte. De diffusie flux in de Fokker-Planck vergelijking voor niet-stationaire condensatie bevat een distributie functie die niet bekend is. Hiervoor wordt als benadering de distributie functie van de stationaire situatie genomen. Het resulterende SDF model is geldig voor alle cluster groottes, rekenefficiënt en toepasbaar voor diverse clusterformatie processen. In het domein van clusters groter dan de kritische cluster grootte wordt de stationaire diffusie flux gegeven door een analytische uitdrukking.

Het Phase Path Analysis (PPA) algoritme is uitgebreid naar $N$-componenten mengsels. 
Hiervoor is de $N$-componenten General Dynamic Equation (NGDE) geconstrueerd. Dit model introduceert clusters op een bron locatie in de $N$-componenten cluster grootte ruimte. De resulterende hyperbolische differentiaal vergelijkingen maken het mogelijk de benadering van de $N$-componenten cluster grootte verdeling zeer efficiënt te berekenen. Voor validatie van de methode is de evolutie van de distributie functie voor een binair mengsel bepaald. Dit laat een zeer goede overeenkomst zien tussen de oplossing van de volledige NBD vergelijkingen en die van de NGDE voor de cluster grootte, cluster compositie en de integraal grootheden van de distributie functie. Het PPA algoritme toegepast op de NGDE geeft een reductie in de benodigde rekentijd met een factor $10^{5}$ vergeleken met de rekentijd die benodigd is voor het oplossen van de NBD vergelijkingen. 


\section{SUMMARY}

This thesis describes efficient solution methods developed for $N$-component condensation processes. These methods are aimed at either the reduction of the numerical effort required for solving the equations describing the condensation process or the simplification of the physical description. The models and corresponding algorithms differ in their ability to describe the condensation phenomenon and the required computing times.

The equilibrium thermodynamics of real gases is presented and a robust numerical procedure is constructed based on Newton-Raphson iteration. The Jacobian of the system of equations has been determined analytically. The initialization scheme for the iterative procedure for these equilibrium problems uses a pressure-extrapolation scheme. Results are given for a three-phase ternary real-gas mixture.

A multigrid method has been developed to enhance the efficiency of implicit numerical methods for solving the $N$-component Becker-Döring (NBD) equations. The geometrical multigrid method for arbitrary number of grid levels is presented. The multigrid algorithm solves the full set of NBD equations 10 times faster than conventional iterative schemes. The method is restricted to the regime of small cluster sizes due to limited available computational resources. However, the time dependent solution of the NBD equations does provide useful insight in the physics of the initial stages of the nucleation process.

For single component condensation the Stationary Diffusion Flux (SDF) model has been derived which is valid in the entire cluster size space. The diffusion flux in the Fokker-Planck equation for unsteady condensation contains an unknown distribution function. This distribution function is approximated by a closed-form expression based on the cluster size distribution function for steady condensation. The resulting Stationary Diffusion Flux model is valid for all cluster sizes, computationally efficient and applicable to various types of cluster formation processes. In the regime of supercritical cluster sizes the diffusion flux is given by an analytical expression.

The Phase Path Analysis (PPA) algorithm has been extended to $N$-component mixtures. For this method the $N$-component General Dynamic Equation (NGDE) is constructed. This model introduces clusters at a source point in the $\mathrm{N}$-component 
cluster size space. The model allows for a very fast solution of the approximate $N$ component cluster size distribution. For validation of the method a nucleation pulse test case involving a binary mixture has been used. Comparison of the numerical results of the NBD equations and those from the NGDE shows excellent agreement for the cluster size, cluster composition and the integral properties of the cluster size distribution. The PPA algorithm applied to the NGDE reduces the computational effort by a factor $10^{5}$ compared to the effort required for solving the full set of NBD equations. 


\section{ACKNOWLEDGMENT}

In the last four years I have been among very pleasant people who helped me in various ways. They have significantly contributed to the completion of this thesis and they deserve to be mentioned.

First of all, I would like to thank my promotor Harry Hoeijmakers for creating the opportunity to carry out this $\mathrm{PhD}$ project at Twister B.V.. You have guided and supported me since I joined the Engineering Fluid Dynamics group and even before during the Solar Challenge project. Your commitment towards students and education is inspiring.

Secondly, I am very grateful to my assistant promotor and daily supervisor Rob Hagmeijer. Rob, I very much enjoyed our collaboration and in my view it was a very fruitful one. Above all, you showed me the fun one can have in doing research. The frequent short visits I payed to the University Twente always gave me new insights and restructured the project. Also, your drive to improve the educational system is a brave conquest and I admire your persistence. Hopefully, we can continue our collaboration in the future.

Although I was not stationed at the University Twente, I supervised several MSc projects. The first student, Mark Klaassen, worked on the inverse design of supersonic condensing nozzles. A very challenging subject and the results were very useful for understanding the physical processes in the Twister Supersonic Separator. Secondly, I had the pleasure to work with Simon Glazenborg who designed a Multigrid method for unary Becker-Döring equations. The project was very successful and lead to a paper, the Kivi Prize and cum laude graduation. It doesn't get much better than that! In this project the help of Kees Venner was indispensable. Kees, I would like to thank you for teaching me Multigrid and for sharing your vision that the physics determines the way a problem can be solved most efficiently. This mind-set should be the basis for any numerical specialist. Furthermore, I am thankful to the rest of the Engineering Fluid Dynamics staff, fellow PhD students and MSc students for the enjoyable discussions over the past four years.

Besides the scientific input from the University Twente, I had the pleasure to work with my colleagues at Twister, most notably Vitaly Kalikmanov. Vitaly, you learned 
theoretical physics the 'old-school' Russian way and your knowledge goes beyond my comprehension. I thank you for teaching me the theoretical part of the physics of phase transitions. I would like to show my gratitude to Marco Betting and Kees Tjeenk Willink for giving me the freedom to execute this PhD project at Twister. During my time at Twister, I was also introduced in the practical/mechanical/chemical side of the gas industry for which I thank all my colleagues. In particular, the 'tubegroup': Bart Prast, Bart Lammers, Roy Ovink and Tijmen Ton. You have provided a very nice and motivating working atmosphere. Tijmen, thank you for sharing the same type of humor we have developed since our joint internship in Brazil. I hope that the last four years I have contributed in the improvement of the physical modeling and leaving the company wasn't an easy choice.

During my stays in Twente, I could always count on the hospitality of Dirk and Marleen. I frequently made a joke about making a reservation at hotel "Tjepkema", but it really felt that way. Thank you! Also, I would like to thank Timo and Jan for their friendship and making my stay a pleasant social event as well.

In my view, the social environment determines largely who you are. Therefore, my family and friends have contributed significantly to my thesis. I want to thank my parents and brothers for their love and patience. Also, I want to acknowledge the continuous interest and support of the Fellows of the DBZ. Our many discussions, due to our different backgrounds, have forced me to put actual problems in a wider perspective.

Finally, I want to thank my wife Dacil. You have shared my happy and frustrating moments and always supported me. Recently, you gave birth to the most beautiful daughter we could wish for. Thank you for your love.

Den Haag, September 2011,

Dennis van Putten 


\section{ABOUT THE AUTHOR}

Dennis van Putten was born on April 12, 1982, in Hoogeveen, The Netherlands, and raised in the small village Hollandscheveld. He attended the "Menso Alting College" in Hoogeveen, from which he graduated in 2001. He then entered the study Mechanical Engineering at the University of Twente. In 2003 he joined the Solar Team Twente and participated in the 2005 World Solar Challenge in Australia. After his return, he started with the master's curriculum in the Engineering Fluid Dynamics group of prof. dr. ir. H.W.M. Hoeijmakers. As part of the curriculum he did an internship at the "Instituto Tecnológico de Aeronáutica" in São José dos Campos, Brazil, in September 2006. Here he worked on the aeroacoustic noise generation of an aircraft in landing configuration. In October 2007 he obtained his master degree (with honors) under supervision of dr. ir. R. Hagmeijer with thesis title: "Reconstruction of the Multi-Component Droplet Size Distribution in Three-Dimensional Condensing Flow".

In November 2007 he started to work as a researcher at Twister B.V. in Rijswijk, The Netherlands, where he worked on the physical modeling of multi-component condensing flows. In January 2008 he and dr. ir. R. Hagmeijer defined a PhD project in collaboration with the University Twente of which the content is described in this thesis. Parts of this work were presented at the $18^{\text {th }}$ International Conference on Nucleation and Atmospheric Aerosols in Prague, Czech Republic (2009), the $7^{\text {th }}$ International Conference on Multiphase Flow in Tampa, USA (2010) and the FERMaTIMPACT-GIMFus Meeting in Sevilla, Spain (2010).

He will continue his career at KEMA Gas Consulting \& Services in Groningen, The Netherlands, and is still active in the Solar Team Twente project. 